\title{
Dimethylzinc-Mediated Alkynylation of Imines
}

\author{
Lorenzo Zani, ${ }^{\dagger}$ Silvia Alesi, ${ }^{\ddagger}$ Pier Giorgio Cozzi, ${ }^{\ddagger}$ and Carsten Bolm ${ }^{\dagger, *}$
}

Institute of Organic Chemistry, RWTH Aachen University, Landoltweg 1, D-52056, Aachen, Germany, and Dipartimento di Chimica “G. Ciamician”, Alma Mater Studiorum, Università di Bologna, Via Selmi 2, I-40126, Bologna, Italy

\section{Table of Contents}

1. General information

2. Alkynylation of $\mathrm{N}$-substituted aromatic imines $\mathbf{1}$ S2

2.1. Typical procedure for the alkynylation of imines $1 \quad$ S2

2.2. Analytical data of compounds 3aa-3oa $\quad$ S2

3. Three component synthesis of propargylic imines $3 \quad$ S8

3.1. Typical procedure for the three component synthesis of propargylic amines $3 \quad$ S8

3.2. Optimization of the reaction conditions $\quad$ S8

3.3. Analytical data of compounds 3pa-3Ba $\quad$ S9

4. Copies of the NMR spectra of compounds 3aa-3na and 3pa-3Ba S13

\section{General Information}

All reactions were carried out under an inert atmosphere of argon or nitrogen, using standard Schlenk techniques. Toluene was purified by destillation over sodium-benzophenone ketyl radical, and $\mathrm{CH}_{2} \mathrm{Cl}_{2}$ was distilled from calcium hydride prior to use. Ethyl acetate, diethyl ether, petroleum ether (PE) and pentane for flash column chromatography were distilled before use. ${ }^{1} \mathrm{H}-\mathrm{NMR}$ spectra were recorded either on a $300 \mathrm{MHz}$ or $400 \mathrm{MHz}$ spectrometer using TMS as internal standard and $\mathrm{CDCl}_{3}$ as solvent, 
unless otherwise stated. ${ }^{13} \mathrm{C}$-NMR spectra were recorded either on a $75 \mathrm{MHz}$ or $100 \mathrm{MHz}$ spectrometer using $\mathrm{CDCl}_{3}$ as solvent, unless otherwise stated. Coupling constants $(J)$ are given in Hertz $(\mathrm{Hz})$. IR absorptions are given in wavenumbers $\left(\mathrm{cm}^{-1}\right)$. Mass peaks are identified by the corresponding $\mathrm{m} / \mathrm{z}$ values. Melting points are reported as intervals and are uncorrected.

\section{Alkynylation of $\mathbf{N}$-substituted aromatic imines 1}

\subsection{Typical procedure for the alkynylation of imines 1 .}

In an oven-dried Schlenk flask under an inert atmosphere of argon alkyne 2a-d (0.75-1.25 mmol, $1.5-$ 2.5 equiv) was dissolved in anhydrous toluene $(4.5 \mathrm{~mL})$. A $2.0 \mathrm{M}$ solution of dimethylzinc in toluene (0.38-0.63 mL, $0.75-1.25 \mathrm{mmol}, 1.5-2.5$ equiv) was then carefully added, and the resulting mixture was stirred at room temperature for $30 \mathrm{~min}$. The appropriate imine $1(0.5 \mathrm{mmol})$ was then added in one portion, and the temperature was increased to the desired value $\left(50-70{ }^{\circ} \mathrm{C}\right)$. The resulting solution was stirred for $24 \mathrm{~h}$, after which a white precipitate appeared in some cases. The reaction was quenched with water $(10 \mathrm{~mL})$. The aqueous phase was extracted with $\mathrm{CH}_{2} \mathrm{Cl}_{2}(3 \times 15 \mathrm{~mL})$ and the organic phase was washed with brine $(25 \mathrm{~mL})$ and dried over $\mathrm{MgSO}_{4}$. Evaporation of the solvent under reduced pressure furnished the crude product typically as a solid, which was purified by flash column chromatography and/or by recrystallization.

\subsection{Analytical data of compounds 3aa-3md.}

$\mathbf{N}$-(p-toluensulfonyl)-3-amino 1,3-diphenylprop-1-yne (3aa). Prepared starting from imine 1a (0.130 g, $0.5 \mathrm{mmol}$ ) and phenylacetylene (2a, $0.077 \mathrm{~g}, 0.75 \mathrm{mmol})$. Purification by flash column chromatography (ethyl acetate/pentane 1:4) afforded pure 3aa. Yield $0.145 \mathrm{~g}(0.4 \mathrm{mmol}, 80 \%)$; white solid; mp 188-189 ${ }^{\circ} \mathrm{C} .{ }^{1} \mathrm{H}-\mathrm{NMR}\left(\mathrm{CDCl}_{3}, 400 \mathrm{MHz}\right) \delta 2.24$ (s, 3H), 4.91 (d, $\left.J=9.2 \mathrm{~Hz}, 1 \mathrm{H}\right), 5.48(\mathrm{~d}, J=$ $9.2 \mathrm{~Hz}, 1 \mathrm{H}), 7.01-7.08(\mathrm{~m}, 2 \mathrm{H}), 7.11-7.34(\mathrm{~m}, 8 \mathrm{H}), 7.44-7.52(\mathrm{~m}, 2 \mathrm{H}), 7.70-7.79$ (m, $2 \mathrm{H}) .{ }^{13} \mathrm{C}-\mathrm{NMR}$ $\left(\mathrm{CDCl}_{3}, 100 \mathrm{MHz}\right) \delta 21.4,49.8,85.4,86.7,121.9,127.3,127.5,128.1,128.4,128.6,128.7,129.5$, 129.7, 131.5, 137.4, 143.5. IR (KBr): 3265, 2223, 1595, 1431, 1327, 1290, 1154, $1047 \mathrm{~cm}^{-1}$. MS (EI, 70 eV): $m / z=360[\mathrm{M}-1]^{+}, 222,206[\mathrm{M}-\mathrm{Ts}]^{+}, 191,105,91,77$. Anal. Calcd. for $\mathrm{C}_{22} \mathrm{H}_{19} \mathrm{NO}_{2} \mathrm{~S}: \mathrm{C}, 73.31 ; \mathrm{H}$, 5.03; N, 3.88. Found: C, 73.09; H, 5.17; N, 3.75.

N-(methansulfonyl)-3-amino 1,3-diphenylprop-1-yne (3ba). Prepared starting from imine $\mathbf{1 b}$ (0.092 g, $0.5 \mathrm{mmol}$ ) and phenylacetylene (2a, $0.077 \mathrm{~g}, 0.75 \mathrm{mmol})$. Purification by flash column 
chromatography (ethyl acetate/pentane 1:4) afforded pure 3ba. Yield 0.112 g (0.39 mmol, 78\%); white solid; mp 100-101 ${ }^{\circ} \mathrm{C} .{ }^{1} \mathrm{H}-\mathrm{NMR}\left(\mathrm{CDCl}_{3}, 400 \mathrm{MHz}\right) \delta 3.00(\mathrm{~s}, 3 \mathrm{H}), 4.92(\mathrm{~d}, J=8.2 \mathrm{~Hz}, 1 \mathrm{H}), 5.56(\mathrm{~d}, J=$ 8.2 Hz, 1H), 7.23-7.36 (m, 6H), 7.37-7.42 (m, 2H), 7.52-7.57 (m, 2H). ${ }^{13} \mathrm{C}-\mathrm{NMR}\left(\mathrm{CDCl}_{3}, 100 \mathrm{MHz}\right) \delta$ 42.0, 49.9, 86.1, 87.1, 121.8,127.4, 128.5, 128.8, 128.9, 129.0, 131.6, 137.2. IR (KBr): 3198, 2199, 1596, 1490, 1445, 1320, 1146, $1054 \mathrm{~cm}^{-1} . \mathrm{MS}(\mathrm{EI}, 70 \mathrm{eV}): \mathrm{m} / \mathrm{z}=285[\mathrm{M}]^{+}, 284[\mathrm{M}-1]^{+}, 222,206[\mathrm{M}-$ $\mathrm{Ms}]^{+}, 191,178,105$, 77. Anal. Calcd. for $\mathrm{C}_{16} \mathrm{H}_{15} \mathrm{NO}_{2} \mathrm{~S}: \mathrm{C}, 67.34 ; \mathrm{H}, 5.30 ; \mathrm{N}, 4.91$. Found: C, 67.02; H, $5.10 ; \mathrm{N}, 4.83$.

N-(2,4,6-trimethylbenzensulfonyl)-3-amino 1,3-diphenylprop-1-yne (3ca). Prepared starting from imine 1c (0.144 g, $0.5 \mathrm{mmol})$ and phenylacetylene (2a, $0.077 \mathrm{~g}, 0.75 \mathrm{mmol})$. Purification by flash column chromatography (ethyl acetate/pentane 1:4) afforded pure 3ca. Yield $0.160 \mathrm{~g}(0.41 \mathrm{mmol}, 82 \%)$; yellow oil that solidified upon standing. ${ }^{1} \mathrm{H}-\mathrm{NMR}\left(\mathrm{CDCl}_{3}, 400 \mathrm{MHz}\right) \delta 2.11(\mathrm{~s}, 3 \mathrm{H}), 2.60(\mathrm{~s}, 6 \mathrm{H}), 4.94$ $(\mathrm{d}, J=8.5 \mathrm{~Hz}, 1 \mathrm{H}), 5.43(\mathrm{~d}, J=8.8 \mathrm{~Hz}, 1 \mathrm{H}), 6.80(\mathrm{~s}, 2 \mathrm{H}), 7.02-7.07(\mathrm{~m}, 2 \mathrm{H}), 7.14-7.31(\mathrm{~m}, 8 \mathrm{H}), 7.45-$ $7.51(\mathrm{~m}, 2 \mathrm{H}) .{ }^{13} \mathrm{C}-\mathrm{NMR}\left(\mathrm{CDCl}_{3}, 100 \mathrm{MHz}\right) \delta 21.0,23.2,49.5,84.9,86.4,122.0,127.3,128.1,128.49$, $128.53,128.7,131.6,131.9,134.4,137.3,139.0,142.2$.

N-(p-nitrobenzensulfonyl)-3-amino 1,3-diphenylprop-1-yne (3da). Prepared starting from imine 1d (0.145 g, $0.5 \mathrm{mmol})$ and phenylacetylene (2a, $0.077 \mathrm{~g}, 0.75 \mathrm{mmol})$. Purification by flash column chromatography (ethyl acetate/pentane 1:4) afforded pure 3da. Yield $0.175 \mathrm{~g}$ (0.45 mmol, 91\%); white solid; mp 167-168 ${ }^{\circ} \mathrm{C} .{ }^{1} \mathrm{H}-\mathrm{NMR}\left(\mathrm{CDCl}_{3}, 400 \mathrm{MHz}\right) \delta 5.20(\mathrm{~d}, J=8.7 \mathrm{~Hz}, 1 \mathrm{H}), 5.64(\mathrm{~d}, J=8.7 \mathrm{~Hz}, 1 \mathrm{H})$, 7.04-7.13 (m, 2H); 7.19-7.41 (m, 6H), 7.49-7.57 (m, 2H), 8.02-8.09 (m, 2H), 8.16-8.23 (m, 2H). ${ }^{13} \mathrm{C}-$ NMR $\left(\mathrm{CDCl}_{3}, 100 \mathrm{MHz}\right) \delta 50.2,84.8,87.6,121.3,124.1,127.4,128.4,128.8,128.9,128.9,129.2$, 131.3, 136.5, 146.2, 149.9. IR (KBr): 3269, 1693, 1605, 1528, 1343, 1310, 1162, $1040 \mathrm{~cm}^{-1}$. MS (EI, 70 $\mathrm{eV}): m / z=391[\mathrm{M}-1]^{+}, 222,206[\mathrm{M}-\mathrm{Ts}]^{+}, 191,128,105,77$. Anal. Calcd. for $\mathrm{C}_{21} \mathrm{H}_{16} \mathrm{~N}_{2} \mathrm{O}_{4} \mathrm{~S}: \mathrm{C}, 64.27$; H, 4.11; N, 7.14. Found: C, 64.18; H, 4.11; N, 6.95.

N-(diphenylphosphoryl)-3-amino 1,3-diphenylprop-1-yne (3ea). Prepared starting from imine 1e (0.122 g, $0.4 \mathrm{mmol})$ and phenylacetylene (2a, $0.061 \mathrm{~g}, 0.6 \mathrm{mmol})$. Purification by flash column chromatography (ethyl acetate/pentane 1:1) afforded pure 3ea. Yield $0.113 \mathrm{~g}$ (0.27 mmol, 69\%); white solid; mp 160-161 ${ }^{\circ} \mathrm{C} .{ }^{1} \mathrm{H}-\mathrm{NMR}\left(\mathrm{CDCl}_{3}, 400 \mathrm{MHz}\right) \delta 3.56(\mathrm{t}, J=9.2 \mathrm{~Hz}, 1 \mathrm{H}), 5.40(\mathrm{t}, J=9.9 \mathrm{~Hz}, 1 \mathrm{H})$, 7.27-7.59 (m, 14H); 7.66-7.74 (m, 2H), 7.81-7.91 (m, 2H), 8.05-8.13 (m, 2H). ${ }^{13} \mathrm{C}-\mathrm{NMR}\left(\mathrm{CDCl}_{3}, 100\right.$ MHz) $\delta 47.2,85.5,88.8,122.6,127.2,127.9,128.1,128.3,128.5,128.6,131.6,131.7,131.8,131.9$, 132.6, 132.7, 133.1, 140.2, 140.2. IR (KBr): 3162, 1961, 1901, 1435, 1188, 1114, $1056 \mathrm{~cm}^{-1}$. MS (EI, 70 
$\mathrm{eV}): \mathrm{m} / \mathrm{z}=407[\mathrm{M}]^{+}, 330\left[\mathrm{M}-\mathrm{C}_{6} \mathrm{H}_{5}\right]^{+}, 206\left[\mathrm{M}-\mathrm{P}(\mathrm{O}) \mathrm{Ph}_{2}\right]^{+}, 191,155,77$. Anal. Calcd. for $\mathrm{C}_{27} \mathrm{H}_{22} \mathrm{NOP}: \mathrm{C}$, 79.59; H, 5.44; N, 3.44. Found: C, 79.75; H, 5.18; N, 3.35.

\section{N-(2-methoxyphenyl)-3-amino 1,3-diphenylprop-1-yne (3ha).}

Prepared starting from imine $\mathbf{1 h}(0.106 \mathrm{~g}, 0.5 \mathrm{mmol})$ and phenylacetylene (2a, $0.128 \mathrm{~g}, 1.25 \mathrm{mmol})$. Purification by flash column chromatography (diethyl ether / PE 1:20) afforded pure 3ha. Yield $0.119 \mathrm{~g}$ (0.38 mmol, 76\%); yellow oil. ${ }^{1} \mathrm{H}-\mathrm{NMR}\left(\mathrm{CDCl}_{3}, 400 \mathrm{MHz}\right) \delta 3.83(\mathrm{~s}, 3 \mathrm{H}), 4.78(\mathrm{~s}, 1 \mathrm{H}), 5.50(\mathrm{~s}, 1 \mathrm{H})$, 6.71-6.91 (m, 4H), 7.23-7.44 (m, 8H), 7.64-7.70 (m, 2H). ${ }^{13} \mathrm{C}-\mathrm{NMR}\left(\mathrm{CDCl}_{3}, 100 \mathrm{MHz}\right) \delta 50.4,55.5$, $84.9,88.7,109.6,111.7,117.7,121.1,122.9,127.4,128.0,128.16,128.20,128.7,131.8,136.4,139.9$, 147.1. IR (neat): 3418, 3062, 2934, 2834, 1600, 1510, 1243, $1223 \mathrm{~cm}^{-1}$. MS (EI, $\left.70 \mathrm{eV}\right): \mathrm{m} / \mathrm{z}=313$ $[\mathrm{M}]^{+}, 282\left[\mathrm{M}-\mathrm{CH}_{3} \mathrm{O}\right]^{+}, 236,225,191\left[\mathrm{M}-\mathrm{C}_{7} \mathrm{H}_{8} \mathrm{NO}\right]^{+}$. Anal. Calcd. for $\mathrm{C}_{22} \mathrm{H}_{19} \mathrm{NO}: \mathrm{C}, 84.31 ; \mathrm{H}, 6.11 ; \mathrm{N}$, 4.47. Found: C, 84.43; H, 6.22; N, 4.20.

N-(p-toluensulfonyl)-1-amino-1-phenylnon-2-yne (3ab) Prepared starting from imine 1a (0.130 g, 0.5 mmol) and 1-octyne (2b, $0.083 \mathrm{~g}, 0.75 \mathrm{mmol})$. Purification by flash column chromatography (ethyl acetate / PE 1:3) afforded pure 3ab. Yield $0.125 \mathrm{~g}$ (0.35 mmol, 69\%); white solid; mp 82-83 ${ }^{\circ} \mathrm{C}$. ${ }^{1} \mathrm{H}-$ NMR $\left(\mathrm{CDCl}_{3}, 400 \mathrm{MHz}\right) \delta 0.81(\mathrm{t}, J=7.0 \mathrm{~Hz}, 3 \mathrm{H}), 1.11-1.28(\mathrm{~m}, 8 \mathrm{H}), 1.89(\mathrm{dt}, J=6.9 \mathrm{~Hz}, J=2.0 \mathrm{~Hz}$, 2H), $2.35(\mathrm{~s}, 3 \mathrm{H}), 4.73(\mathrm{dd}, J=8.8 \mathrm{~Hz}, J=2.0 \mathrm{~Hz}, 1 \mathrm{H}), 5.23(\mathrm{~d}, J=8.8 \mathrm{~Hz}, 1 \mathrm{H}), 7.17-7.27(\mathrm{~m}, 5 \mathrm{H})$; 7.37-7.43 (m, 2H), 7.67-7.73 (m, 2H). ${ }^{13} \mathrm{C}-\mathrm{NMR}\left(\mathrm{CDCl}_{3}, 100 \mathrm{MHz}\right) \delta 14.1,18.6,21.6,22.6,28.3,28.6$, 31.3, 49.5, 76.6, 87.6, 127.2, 127.4, 128.1, 128.4, 129.3, 137.5, 138.0, 143.1. IR (KBr): 3288, 2928, 2857, 2227, 1597, 1330, 1157, $1031 \mathrm{~cm}^{-1}$. MS (EI, $\left.70 \mathrm{eV}\right): \mathrm{m} / \mathrm{z}=299,214[\mathrm{M}-\mathrm{Ts}]^{+}, 155,143,91,77$. Anal. Calcd. for $\mathrm{C}_{22} \mathrm{H}_{27} \mathrm{NO}_{2} \mathrm{~S}: \mathrm{C}, 71.51 ; \mathrm{H}, 7.36 ; \mathrm{N}, 3.79$. Found: C, 71.65; H, 7.70; N, 3.66.

N-(p-toluensulfonyl)-3-amino-3-phenyl-1-trimethylsilylprop-1-yne (3ac). Prepared starting from imine 1a $(0.130 \mathrm{~g}, 0.5 \mathrm{mmol})$ and trimethylsilylethyne (2c, $0.075 \mathrm{~g}, 0.75 \mathrm{mmol})$. Purification by flash column chromatography (ethyl acetate / PE 1:5) afforded pure 3ac. Yield $0.091 \mathrm{~g}$ (0.26 mmol, 51\%); white solid; mp 139-140 ${ }^{\circ} \mathrm{C} .{ }^{1} \mathrm{H}-\mathrm{NMR}\left(\mathrm{CDCl}_{3}, 400 \mathrm{MHz}\right) \delta 0.04(\mathrm{~s}, 9 \mathrm{H}), 2.43(\mathrm{~s}, 3 \mathrm{H}), 4.81(\mathrm{~d}, J=8.5$ $\mathrm{Hz}, 1 \mathrm{H}), 5.34(\mathrm{~d}, J=9.2 \mathrm{~Hz}, 1 \mathrm{H}), 7.25-7.36(\mathrm{~m}, 5 \mathrm{H}), 7.46-7.52(\mathrm{~m}, 2 \mathrm{H}), 7.76-7.82(\mathrm{~m}, 2 \mathrm{H}) .{ }^{13} \mathrm{C}-\mathrm{NMR}$ $\left(\mathrm{CDCl}_{3}, 100 \mathrm{MHz}\right) \delta-0.3,21.6,49.7,91.6,101.4,127.2,127.4,128.3,128.5,129.5,137.2,137.3$, 143.3. IR (KBr): 3256, 2175, 1952, 1911, 1425, 1328, 1249, 1162, $1056 \mathrm{~cm}^{-1}$. MS (EI, $\left.70 \mathrm{eV}\right): \mathrm{m} / \mathrm{z}=$ $356[\mathrm{M}-1]^{+}, 260,234,218,202$ [M-Ts] ${ }^{+}, 159,91$. Anal. Calcd. for $\mathrm{C}_{19} \mathrm{H}_{23} \mathrm{NO}_{2} \mathrm{SSi}: \mathrm{C}, 63.83 ; \mathrm{H}, 6.48 ; \mathrm{N}$, 3.92. Found: C, 63.75; H, 6.35; N, 3.78. 
N-(p-toluensulfonyl)-3-amino-3-phenyl-1-(4-trifluoromethyl)phenylprop-1-yne (3ad). Prepared starting from imine 1a $(0.130 \mathrm{~g}, 0.5 \mathrm{mmol})$ and 4-(trifluoromethyl)phenylacetylene (2d, $0.128 \mathrm{~g}, 0.75$ mmol). Purification by flash column chromatography (ethyl acetate / PE 1:4) afforded pure 3ad. Yield $0.183 \mathrm{~g}$ (0.43 mmol, 86\%); white solid; mp 140-141 ${ }^{\circ} \mathrm{C} .{ }^{1} \mathrm{H}-\mathrm{NMR}\left(\mathrm{CDCl}_{3}, 300 \mathrm{MHz}\right) \delta 2.24(\mathrm{~s}, 3 \mathrm{H})$, $4.96(\mathrm{~d}, J=8.9 \mathrm{~Hz}, 1 \mathrm{H}), 5.50(\mathrm{~d}, J=9.1 \mathrm{~Hz}, 1 \mathrm{H}), 7.12-7.34(\mathrm{~m}, 7 \mathrm{H}), 7.40-7.49(\mathrm{~m}, 4 \mathrm{H}), 7.70-7.77$ (m, 2H). ${ }^{13} \mathrm{C}-\mathrm{NMR}\left(\mathrm{CDCl}_{3}, 75 \mathrm{MHz}\right) \delta 21.4,49.7,85.2,88.1,125.0,125.1,125.8,127.3,127.5,128.6$, 128.7, 128.8, 129.6, 131.8, 136.9, 137.4, 143.6. IR (KBr): 3278, 1924, 1807, 1736, 1606, 1328, 1163 $\mathrm{cm}^{-1}$. MS (EI, $\left.70 \mathrm{eV}\right): m / z=410[\mathrm{M}-\mathrm{F}]^{+}, 274[\mathrm{M}-\mathrm{Ts}]^{+}, 259$, 173, 91, 77. Anal. Calcd. for $\mathrm{C}_{23} \mathrm{H}_{18} \mathrm{NO}_{2} \mathrm{SF}_{3}$ : C, 64.33; H, 4.22; N, 3.26. Found: C, 63.99; H, 4.69; N, 3.25. HRMS (EI): $\mathrm{m} / \mathrm{z}$ calcd. For $\mathrm{C}_{23} \mathrm{H}_{18} \mathrm{NO}_{2} \mathrm{SF}_{3}-\mathrm{SO}_{2} \mathrm{H}: 364.1313$. Found: 364.1313 .

N-(p-nitrobenzensulfonyl)-1-amino-1-phenylnon-2-yne (3db). Prepared starting from imine 1d $(0.145 \mathrm{~g}, 0.5 \mathrm{mmol})$ and 1-octyne $(2 \mathbf{b}, 0.083 \mathrm{~g}, 0.75 \mathrm{mmol})$. Purification by flash column chromatography (ethyl acetate/PE 1:4), followed by recrystallization from MTBE afforded pure 3db. Yield $0.159 \mathrm{~g}(0.39 \mathrm{mmol}, 79 \%)$; yellow solid; mp 103-104 ${ }^{\circ} \mathrm{C} .{ }^{1} \mathrm{H}-\mathrm{NMR}\left(\mathrm{CDCl}_{3}, 300 \mathrm{MHz}\right) \delta 0.87(\mathrm{t}, J$ $=6.9 \mathrm{~Hz}, 3 \mathrm{H}), 1.14-1.36(\mathrm{~m}, 8 \mathrm{H}), 1.96(\mathrm{dt}, J=6.9 \mathrm{~Hz}, J=2.1 \mathrm{~Hz}, 2 \mathrm{H}), 5.04(\mathrm{~d}, J=7.8 \mathrm{~Hz}, 1 \mathrm{H}), 5.39$ $(\mathrm{d}, J=8.2 \mathrm{~Hz}, 1 \mathrm{H}), 7.27-7.36(\mathrm{~m}, 3 \mathrm{H}), 7.39-7.48(\mathrm{~m}, 2 \mathrm{H}), 7.97-8.07(\mathrm{~m}, 2 \mathrm{H}), 8.25-8.34(\mathrm{~m}, 2 \mathrm{H}) .{ }^{13} \mathrm{C}-$ $\mathrm{NMR}\left(\mathrm{CDCl}_{3}, 75 \mathrm{MHz}\right) \delta 14.0,18.5,22.5,28.2,28.3,28.5,31.2,49.9,76.1,88.3,123.9,127.3,128.6$; 128.7, 128.7, 137.3, 146.4, 149.9. IR (KBr): 3268, 2927, 2230, 1602, 1525, 1345, 1309, $1166,1044 \mathrm{~cm}^{-}$ 1. $\mathrm{MS}(\mathrm{EI}, 70 \mathrm{eV}): \mathrm{m} / \mathrm{z}=330,291\left[\mathrm{M}-\mathrm{C}_{8} \mathrm{H}_{15}\right]^{+}, 214[\mathrm{M}-\mathrm{Ns}]^{+}, 143$, 91, 77. Anal. Calcd. for $\mathrm{C}_{21} \mathrm{H}_{24} \mathrm{~N}_{2} \mathrm{SO}_{4}$ : C, 62.98; H, 6.04; N, 6.99. Found: C, 63.38; H, 6.06; N, 6.94.

N-(p-toluensulfonyl)-3-amino-3-(4-methylphenyl)-1-phenylprop-1-yne (3ia). Prepared starting from imine 1i (0.109 g, $0.4 \mathrm{mmol})$ and phenylacetylene (2a, $0.061 \mathrm{~g}, 0.75 \mathrm{mmol})$. Purification by flash column chromatography (ethyl acetate / PE 1:4) afforded pure 3ia. Yield 0.116 g (0.31 mmol, 77\%); white solid; mp 190-191 ${ }^{\circ} \mathrm{C} .{ }^{1} \mathrm{H}-\mathrm{NMR}\left(\mathrm{CDCl}_{3}, 400 \mathrm{MHz}\right) \delta 2.32$ (s, 3H), 2.34 (s, 3H), 4.89 (d, $J=9.1$ $\mathrm{Hz}, 1 \mathrm{H}), 5.52$ (d, $J=9.1 \mathrm{~Hz}, 1 \mathrm{H}), 7.07-7.17$ (m, 4H), 7.19-7.33 (m, 5H), 7.38-7.46 (m, 2H), 7.77-7.85 (m, 2H). ${ }^{13} \mathrm{C}-\mathrm{NMR}\left(\mathrm{CDCl}_{3}, 100 \mathrm{MHz}\right) \delta 21.2,21.5,49.6,85.7,86.5,122.0,127.1,127.4,128.0,128.4$, 129.3, 129.4, 131.4, 134.4, 137.3, 138.2, 143.4. IR (KBr): 3269, 2221, 1430, 1329, 1155, $1047 \mathrm{~cm}^{-1}$. MS (EI, $70 \mathrm{eV}): m / z=310,236,220[\mathrm{M}-\mathrm{Ts}]^{+}, 205,128,105,91,77$. HRMS (EI): $m / z$ calcd. For $\mathrm{C}_{23} \mathrm{H}_{21} \mathrm{NO}_{2} \mathrm{~S}-\mathrm{SO}_{2} \mathrm{H}: 310.1596$. Found: 310.1595 . 
N-(p-toluensulfonyl)-1-amino-1-(4-methylphenyl)non-2-yne (3ib). Prepared starting from imine 1i $(0.109 \mathrm{~g}, 0.4 \mathrm{mmol})$ and 1-octyne (2, $0.066 \mathrm{~g}, 0.6 \mathrm{mmol})$. Purification by flash column chromatography

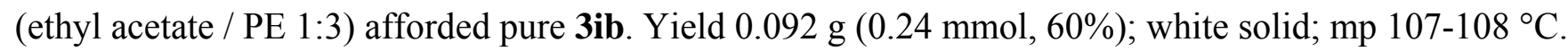
${ }^{1} \mathrm{H}-\mathrm{NMR}\left(\mathrm{CDCl}_{3}, 300 \mathrm{MHz}\right) \delta 0.88(\mathrm{t}, J=6.9 \mathrm{~Hz}, 3 \mathrm{H}), 1.16-1.36(\mathrm{~m}, 8 \mathrm{H}), 1.95(\mathrm{dt}, J=6.9 \mathrm{~Hz}, J=2.1$ Hz, 2H), 2.32 (s, 3H), 2.43 (s, 3H), 4.72 (d, $J=8.7 \mathrm{~Hz}, 1 \mathrm{H}), 5.26$ (d, J=8.7 Hz, 1H), 7.06-7.16 (m, 2H), 7.24-7.37 (m, 4H), 7.73-7.81 (m, 2H). ${ }^{13} \mathrm{C}-\mathrm{NMR}\left(\mathrm{CDCl}_{3}, 75 \mathrm{MHz}\right) \delta 14.0,18.5,21.1,21.5,22.5$, $28.3,28.5 ; 31.3,49.2,76.8,87.3,127.2,127.5,129.2,129.3,135.2,137.6,138.0,143.2$. IR (KBr): $3271,2930,2224,1916,1334,1160,1028 \mathrm{~cm}^{-1}$. MS (EI, $\left.70 \mathrm{eV}\right): \mathrm{m} / \mathrm{z}=313,274\left[\mathrm{M}-\mathrm{C}_{8} \mathrm{H}_{15}\right]^{+}, 228[\mathrm{M}-$ $\mathrm{Ts}]^{+}, 157,105,91,77$. Anal. Calcd. for $\mathrm{C}_{23} \mathrm{H}_{29} \mathrm{NO}_{2} \mathrm{~S}: \mathrm{C}, 72.03 ; \mathrm{H}, 7.62 ; \mathrm{N}, 3.65$. Found: C, 71.64; H, $7.76 ; \mathrm{N}, 3.73$.

N-(p-toluensulfonyl)-3-amino-3-(4-methoxyphenyl)-1-phenylprop-1-yne (3ja). Prepared starting from imine 1j (0.145 g, $0.5 \mathrm{mmol})$ and phenylacetylene (2a, $0.077 \mathrm{~g}, 0.75 \mathrm{mmol})$. Purification by flash column chromatography (ethyl acetate / PE 1:3) afforded pure 3ja. Yield 0.171 g (0.44 mmol, 87\%); white solid; mp 186-187 ${ }^{\circ} \mathrm{C} .{ }^{1} \mathrm{H}-\mathrm{NMR}\left(\mathrm{CDCl}_{3}, 400 \mathrm{MHz}\right) \delta 2.32$ (s, 3H), 3.80 (s, 3H), 4.90 (d, $J=8.9$ $\mathrm{Hz}, 1 \mathrm{H}), 5.51(\mathrm{~d}, J=9.0 \mathrm{~Hz}, 1 \mathrm{H}), 6.84-6.90$ (m, 2H), 7.08-7.15 (m, 2H), 7.20-7.33 (m, 5H), 7.43-7.50 $(\mathrm{m}, 2 \mathrm{H}), 7.78-7.84(\mathrm{~m}, 2 \mathrm{H}) .{ }^{13} \mathrm{C}-\mathrm{NMR}\left(\mathrm{CDCl}_{3}, 100 \mathrm{MHz}\right) \delta 21.5,49.3,55.4,85.7,86.5,114.0,121.9$, 127.4, 128.0, 128.4, 128.5, 129.4, 129.4, 131.4, 137.3, 143.4, 159.5. IR (KBr): 3263, 2221, 1509, 1329, 1250, 1157, $1034 \mathrm{~cm}^{-1}$. MS (EI, $\left.70 \mathrm{eV}\right): \mathrm{m} / \mathrm{z}=391[\mathrm{M}]^{+}, 326,236[\mathrm{M}-\mathrm{Ts}]^{+}, 235,221,178,91,77$. Anal. Calcd. for $\mathrm{C}_{23} \mathrm{H}_{21} \mathrm{NO}_{3} \mathrm{~S}$ : C, 70.56; H, 5.41; N, 3.58. Found: C, 70.42; H, 5.36; N, 3.57.

N-(p-toluensulfonyl)-3-amino-3-(2-naphtyl)-1-phenylprop-1-yne (3ka). Prepared starting from imine 1k (0.143 g, $0.5 \mathrm{mmol})$ and phenylacetylene (2a, $0.077 \mathrm{~g}, 0.75 \mathrm{mmol})$. Purification by recrystallization

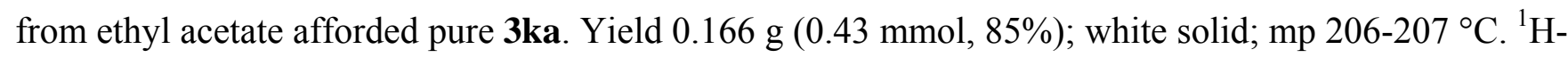
$\operatorname{NMR}\left(\mathrm{CD}_{2} \mathrm{Cl}_{2}, 400 \mathrm{MHz}\right) \delta 2.21(\mathrm{~s}, 3 \mathrm{H}), 5.06(\mathrm{~d}, J=9.2 \mathrm{~Hz}, 1 \mathrm{H}), 5.60(\mathrm{~d}, J=9.2 \mathrm{~Hz}, 1 \mathrm{H}), 7.07-7.30$ $(\mathrm{m}, 7 \mathrm{H}), 7.39-7.47(\mathrm{~m}, 2 \mathrm{H}), 7.51-7.57(\mathrm{~m}, 1 \mathrm{H}), 7.68-7.81(\mathrm{~m}, 5 \mathrm{H}), 7.90(\mathrm{~s}, 1 \mathrm{H}) .{ }^{13} \mathrm{C}-\mathrm{NMR}\left(\mathrm{CD}_{2} \mathrm{Cl}_{2}, 100\right.$ $\mathrm{MHz}) \delta 21.2,50.0,85.4,86.7,125.0,126.0,126.1,126.4,126.5,127.4,127.5,128.0,128.1,128.6$, 128.6, 129.5, 131.5, 133.0, 133.1, 134.8, 137.2, 143.8. IR (KBr): 3259, 2220, 1427, 1329, 1156, 1039 $\mathrm{cm}^{-1}$. MS (EI, $\left.70 \mathrm{eV}\right): \mathrm{m} / \mathrm{z}=411[\mathrm{M}]^{+}, 346,272,256[\mathrm{M}-\mathrm{Ts}]^{+}, 241,228,155,128,91$, 77. Anal. Calcd. for $\mathrm{C}_{26} \mathrm{H}_{21} \mathrm{NO}_{2} \mathrm{~S}$ : C, 75.89; H, 5.14; N, 3.40. Found: C, 75.95; H, 5.10; N, 3.36.

N-(p-toluensulfonyl)-3-amino-3-(2-bromophenyl)-1-phenylprop-1-yne (3la). Prepared starting from imine $1 \mathrm{l}$ (0.169 g, $0.5 \mathrm{mmol})$ and phenylacetylene (2a, $0.077 \mathrm{~g}, 0.75 \mathrm{mmol})$. Purification by flash 
column chromatography (ethyl acetate / PE 1:4) afforded pure 3la. Yield 0.205 g (0.47 mmol, 93\%); white solid; mp 169-170 ${ }^{\circ} \mathrm{C} .{ }^{1} \mathrm{H}-\mathrm{NMR}\left(\mathrm{CDCl}_{3}, 300 \mathrm{MHz}\right) \delta 2.24(\mathrm{~s}, 3 \mathrm{H}), 5.10(\mathrm{~d}, J=8.3 \mathrm{~Hz}, 1 \mathrm{H}), 5.75$ $(\mathrm{d}, J=8.3 \mathrm{~Hz}, 1 \mathrm{H}), 7.04-7.26(\mathrm{~m}, 9 \mathrm{H}), 7.43-7.52(\mathrm{~m}, 2 \mathrm{H}), 7.67-7.74(\mathrm{~m}, 2 \mathrm{H}) .{ }^{13} \mathrm{C}-\mathrm{NMR}\left(\mathrm{CD}_{2} \mathrm{Cl}_{2}, 75\right.$ MHz) $\delta 21.4,50.1,85.0,86.5,121.9,123.0,127.5,127.8,128.1,128.6,129.4,129.6,130.0,131.6$, 133.5, 136.7, 137.2, 143.5. IR (KBr): 3268, 1923, 1594, 1329, 1156, $1054 \mathrm{~cm}^{-1}$. MS (EI, $\left.70 \mathrm{eV}\right): \mathrm{m} / \mathrm{z}=$ $441[\mathrm{M}]^{+}, 439,378,376,302,300,286[\mathrm{M}-\mathrm{Ts}]^{+}, 284,204,105,91$, 77. Anal. Calcd. for $\mathrm{C}_{22} \mathrm{H}_{18} \mathrm{NO}_{2} \mathrm{BrS}$ : C, 60.01; H, 4.12; N, 3.18. Found: C, 60.05; H, 4.48; N, 3.05.

N-(p-toluensulfonyl)-3-amino-3-(2-furyl)-1-phenylprop-1-yne (3ma). Prepared starting from imine $1 \mathrm{~m}(0.125 \mathrm{~g}, 0.5 \mathrm{mmol})$ and phenylacetylene (2a, $0.077 \mathrm{~g}, 0.75 \mathrm{mmol})$. Purification by flash column chromatography (ethyl acetate / PE 2:7) afforded pure 3ma. Yield $0.140 \mathrm{~g}$ (0.40 mmol, 80\%); pale brown solid; mp 151-152 ${ }^{\circ} \mathrm{C} .{ }^{1} \mathrm{H}-\mathrm{NMR}\left(\mathrm{CDCl}_{3}, 400 \mathrm{MHz}\right) \delta 2.25(\mathrm{~s}, 3 \mathrm{H}), 4.98(\mathrm{~d}, \mathrm{~J}=9.0 \mathrm{~Hz}, 1 \mathrm{H}), 5.53$ $(\mathrm{d}, J=9.0 \mathrm{~Hz}, 1 \mathrm{H}), 6.22(\mathrm{dd}, J=3.3 \mathrm{~Hz}, J=1.9 \mathrm{~Hz}, 1 \mathrm{H}), 6.31(\mathrm{~d}, J=3.3 \mathrm{~Hz}, 1 \mathrm{H}), 7.05-7.29(\mathrm{~m}, 8 \mathrm{H})$, 7.69-7.76 (m, 2H). ${ }^{13} \mathrm{C}-\mathrm{NMR}\left(\mathrm{CD}_{2} \mathrm{Cl}_{2}, 100 \mathrm{MHz}\right) \delta 21.5,44.2,83.4,85.3,108.3,110.4,121.6,127.3$, 128.0, 128.6, 129.4, 131.5, 137.2, 143.1, 143.5, 149.4. IR (KBr): 3263, 2225, 1433, 1333, 1157, 1039 $\mathrm{cm}^{-1}$. MS (EI, $\left.70 \mathrm{eV}\right): \mathrm{m} / \mathrm{z}=303,250\left[\mathrm{M}-\mathrm{C}_{8} \mathrm{H}_{5}\right]^{+}, 196[\mathrm{M}-\mathrm{Ts}]^{+}, 181\left[\mathrm{M}-\mathrm{C}_{7} \mathrm{H}_{8} \mathrm{NO}_{2} \mathrm{~S}\right]^{+}, 168,152,105,91$, 77. Anal. Calcd. for $\mathrm{C}_{20} \mathrm{H}_{17} \mathrm{NO}_{3} \mathrm{~S}$ : C, 68.35; H, 4.88; N, 3.89. Found: C, 68.00; H, 5.20; N, 3.89.

N-(p-toluensulfonyl)-3-amino-3-(2-furyl)-1-(4-trifluoromethylphenyl) prop-1-yne (3md). Prepared starting from imine $\mathbf{1 m}(0.125 \mathrm{~g}, 0.5 \mathrm{mmol})$ and 4-(trifluoromethyl)phenylacetylene (2d, $0.128 \mathrm{~g}, 0.75$ mmol). Purification by recrystallization from MTBE afforded pure 3md. Yield $0.199 \mathrm{~g}(0.47 \mathrm{mmol}$, 95\%); white solid; mp 117-118 ${ }^{\circ} \mathrm{C} .{ }^{1} \mathrm{H}-\mathrm{NMR}\left(\mathrm{CDCl}_{3}, 400 \mathrm{MHz}\right) \delta 2.25$ (s, 3H), 5.06 (d, $J=8.9 \mathrm{~Hz}$, $1 \mathrm{H}), 5.55$ (d, $J=8.9 \mathrm{~Hz}, 1 \mathrm{H}), 6.24(\mathrm{dd}, J=3.2 \mathrm{~Hz}, J=1.9 \mathrm{~Hz}, 1 \mathrm{H}), 6.30(\mathrm{~d}, J=3.2 \mathrm{~Hz}, 1 \mathrm{H}), 7.12-7.31$ $(\mathrm{m}, 5 \mathrm{H}), 7.41-7.48(\mathrm{~m}, 2 \mathrm{H}), 7.69-7.76(\mathrm{~m}, 2 \mathrm{H}) .{ }^{13} \mathrm{C}-\mathrm{NMR}\left(\mathrm{CD}_{2} \mathrm{Cl}_{2}, 100 \mathrm{MHz}\right) \delta 21.5,44.0,83.9,86.0$, $108.5,110.5,125.0,125.0,125.4,127.4,129.5,131.8,137.2,143.2,143.6,148.8$. IR (KBr): 3270 , 2923, 1926, 1616, 1329, 1162, $1132 \mathrm{~cm}^{-1}$. MS (EI, $\left.70 \mathrm{eV}\right): \mathrm{m} / \mathrm{z}=400,354,280,264$ [M-Ts] $, 249,139$, 91, 77. Anal. Calcd. for $\mathrm{C}_{21} \mathrm{H}_{16} \mathrm{NO}_{3} \mathrm{SF}_{3}$ : C, 60.14; H, 3.85; N, 3.34. Found: C, 60.03; H, 4.15; N, 3.32.

N-(p-toluensulfonyl)-3-amino-3-cyclohexyl-1-phenylprop-1-yne (3na). Prepared starting from imine 1n $(0.133 \mathrm{~g}, 0.5 \mathrm{mmol})$ and phenylacetylene (2a, $0.077 \mathrm{~g}, 0.75 \mathrm{mmol})$. Purification by recrystallization from EtOAc afforded pure 3na. Yield $0.155 \mathrm{~g}$ (0.42 mmol, 84\%); white solid; mp 202-203 ${ }^{\circ} \mathrm{C} .{ }^{1} \mathrm{H}-\mathrm{NMR}$ $\left(\mathrm{CDCl}_{3}, 400 \mathrm{MHz}\right) \delta 0.95-1.23(\mathrm{~m}, 5 \mathrm{H}), 1.66-1.87(\mathrm{~m}, 6 \mathrm{H}), 2.25(\mathrm{~s}, 3 \mathrm{H}), 4.04(\mathrm{dd}, J=9.9 \mathrm{~Hz}, J=6.0$ $\mathrm{Hz}, 1 \mathrm{H}), 4.59$ (d, $J=9.9 \mathrm{~Hz}, 1 \mathrm{H}), 6.93-7.00(\mathrm{~m}, 2 \mathrm{H}), 7.11-7.22(\mathrm{~m}, 5 \mathrm{H}), 7.70-7.76(\mathrm{~m}, 2 \mathrm{H}) .{ }^{13} \mathrm{C}-\mathrm{NMR}$ 
$\left(\mathrm{CD}_{2} \mathrm{Cl}_{2}, 100 \mathrm{MHz}\right) \delta 21.6,25.86,25.94,26.3,28.6,29.3,43.3,51.6,85.2,86.2,122.2,127.5,128.0$, 128.3, 129.5, 131.5, 137.4, 143.4. IR (KBr): 3853, 3744, 3452, 3279, 2925, 2360, 2337, 1650, 1331,

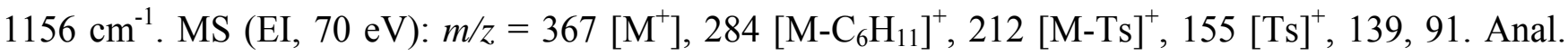
Calcd. for $\mathrm{C}_{22} \mathrm{H}_{25} \mathrm{NO}_{2} \mathrm{~S}$ : C, 71.90; H, 6.86; N, 3.81. Found: C, 71.54; H, 7.14; N, 3.75 .

N-(p-toluensulfonyl)-3-amino-5-methyl-1-phenylhex-1-yne (3oa). Prepared starting from imine 1o $(0.120 \mathrm{~g}, 0.5 \mathrm{mmol})$ and phenylacetylene (2a, $0.077 \mathrm{~g}, 0.75 \mathrm{mmol})$. Purification by flash column chromatography (ethyl acetate / PE 1:7) afforded pure 3na. Yield 0.025 g (0.08 mmol, 15\%); white solid. ${ }^{1} \mathrm{H}-\mathrm{NMR}\left(\mathrm{CDCl}_{3}, 400 \mathrm{MHz}\right) \delta 0.90(\mathrm{t}, J=6.5 \mathrm{~Hz}, 6 \mathrm{H}), 1.16-1.29(\mathrm{~m}, 1 \mathrm{H}), 1.50-1.64(\mathrm{~m}, 2 \mathrm{H})$, 2.27 (s, 3H), 4.28 (dt, $J=9.6 \mathrm{~Hz}, J=7.7 \mathrm{~Hz}, 1 \mathrm{H}), 4.51$ (d, $J=9.6 \mathrm{~Hz}, 1 \mathrm{H}), 6.95-7.00$ (m, 2H), 7.13$7.28(\mathrm{~m}, 5 \mathrm{H}), 7.74-7.80(\mathrm{~m}, 2 \mathrm{H}) .{ }^{13} \mathrm{C}-\mathrm{NMR}\left(\mathrm{CD}_{2} \mathrm{Cl}_{2}, 100 \mathrm{MHz}\right) \delta 21.5,22.3,22.4,24.9,44.9,45.8$, $84.3,87.4,127.5,128.0,128.3,129.5,131.4,135.8,137.4,143.4$.

\section{Three-component synthesis of propargylic imines 3}

\subsection{Typical procedure for the three-component synthesis of propargylic imines 3}

METHOD A. In an oven-dried Schlenk flask under an inert atmosphere of argon were placed the appropriate aldehyde $7(0.2 \mathrm{mmol})$ and 2-methoxyaniline (6, $0.2 \mathrm{mmol}, 1.0$ equiv), followed by anhydrous toluene $(2.0 \mathrm{~mL})$. After 30 min stirring, a $2.0 \mathrm{M}$ solution of dimethylzinc in toluene $(0.35 \mathrm{~mL}$, $0.7 \mathrm{mmol}, 3.5$ equiv) was added. The reaction mixture was then stirred for another $30 \mathrm{~min}$, before adding phenylacetylene (2a, $0.5 \mathrm{mmol}, 2.5$ equiv). The resulting solution was stirred at room temperature for 48-60 h. The reaction mixture was diluted with diethyl ether $(5 \mathrm{~mL})$, and quenched with water $(10 \mathrm{~mL})$. The resulting heterogeneous mixture was filtered over Celite ${ }^{\circledR}$. The aqueous phase was separated and washed with diethylether $(2 \times 5 \mathrm{~mL})$. The combined organic layers were dried over $\mathrm{Na}_{2} \mathrm{SO}_{4}$ and evaporated under reduced pressure to give the crude product, which was then purified by flash column chromatography (see Supporting Information for details).

METHOD B (concentrated conditions). In an oven-dried Schlenk flask under an inert atmosphere of argon were placed the appropriate aldehyde $7(0.4 \mathrm{mmol})$ and 2-methoxyaniline $(6,0.4 \mathrm{mmol}, 1.0$ equiv). A $2.0 \mathrm{M}$ solution of dimethylzinc in toluene $(0.7 \mathrm{~mL}, 1.4 \mathrm{mmol}, 3.5$ equiv) was immediately added. The reaction mixture was then stirred for $15 \mathrm{~min}$, before the addition of phenylacetylene (1a, 1.0 mmol, 2.5 equiv). The resulting solution was stirred at room temperature for $48-96 \mathrm{~h}$. Subsequently, the protocol followed the procedure reported above for Method A. 


\subsection{Optimization of the reaction conditions (Method A)}

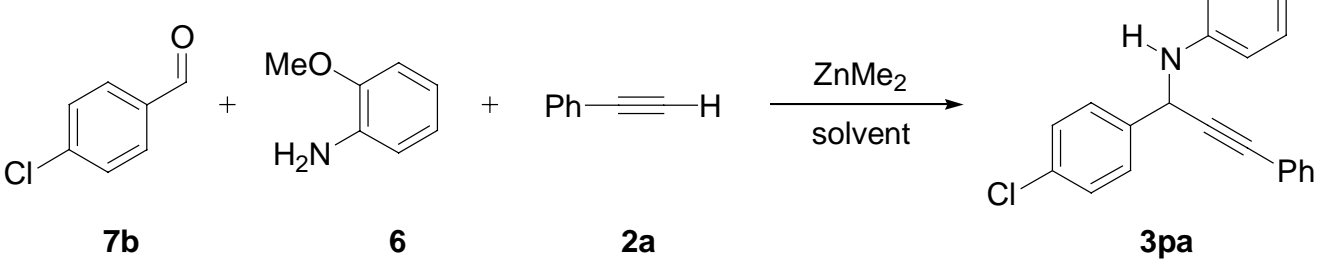

\begin{tabular}{ccccc}
\hline entry & solvent & $\mathrm{Me}_{2} \mathrm{Zn}$ (equiv) & $\begin{array}{c}\text { phenylacetylene } \\
\text { (2a, equiv) }\end{array}$ & yield (\%) \\
\hline 1 & $\mathrm{CH}_{2} \mathrm{Cl}_{2}$ & 2.5 & 1.5 & 32 \\
2 & $\mathrm{CH}_{2} \mathrm{Cl}_{2}$ & 3.5 & 2.5 & 72 \\
3 & $\mathrm{CH}_{2} \mathrm{Cl}_{2}$ & 4.5 & 3.0 & 53 \\
4 & Toluene & 3.5 & 2.5 & 76 \\
5 & $\mathrm{THF}$ & 3.5 & 2.5 & 0 \\
\hline
\end{tabular}

\subsection{Analytical data of compounds 3pa-3Ba.}

N-(2-methoxyphenyl)-3-amino-3-(4-chlorophenyl)-1-phenylprop-1-yne (3pa). Prepared starting from aldehyde $7 \mathbf{b}(0.030 \mathrm{~g}, 0.2 \mathrm{mmol})$ and phenylacetylene (2a, $0.051 \mathrm{~g}, 0.5 \mathrm{mmol})$ according to Method A. Purification by flash column chromatography $\left(\mathrm{CH}_{2} \mathrm{Cl}_{2} /\right.$ cyclohexane 2:3) afforded pure 3pa. Yield $0.053 \mathrm{~g}$ (0.15 mmol, 76\%); yellow oil. ${ }^{1} \mathrm{H}-\mathrm{NMR}\left(\mathrm{CDCl}_{3}, 400 \mathrm{MHz}\right) \delta 3.83(\mathrm{~s}, 3 \mathrm{H}), 4.81$ (brs, $\left.1 \mathrm{H}\right)$, 5.43(s, 1H), 6.78-6.85 (m, 4H), 7.26-7.33 (m, 3H), 7.36 (d, J=8.1 Hz, 2H), 7.42-7.45 (m, 2H), 7.62 (d, $J=8.1 \mathrm{~Hz}, 2 \mathrm{H}) .{ }^{13} \mathrm{C}-\mathrm{NMR}\left(\mathrm{CDCl}_{3}, 100 \mathrm{MHz}\right) \delta 49.8,55.4,85.1,88.1,109.5,111.6,117.9,121.0,122.6$, 128.2, 128.4, 128.6, 128.8, 131.7, 133.7, 136.1, 138.5, 147.1. IR (neat): 3409, 3058, 2945, 2939, 1606, 1507, 1480, $1023 \mathrm{~cm}^{-1}$. Anal. Calcd. for $\mathrm{C}_{22} \mathrm{H}_{18} \mathrm{ClNO}$ : C, 75.97; H, 5.22; N, 4.03. Found: C, 75.80; H, $5.29 ; \mathrm{N}, 4.02$.

N-(2-methoxyphenyl)-3-amino-3-(3-bromophenyl)-1-phenylprop-1-yne (3qa). Prepared starting from aldehyde 7c $(0.037 \mathrm{~g}, 0.2 \mathrm{mmol})$ and phenylacetylene (2a, $0.051 \mathrm{~g}, 0.5 \mathrm{mmol})$ according to Method A. Purification by flash column chromatography (diethyl ether / cyclohexane 1:9) afforded pure 
3qa. Yield $0.057 \mathrm{~g}(0.15 \mathrm{mmol}, 73 \%)$; yellow oil. ${ }^{1} \mathrm{H}-\mathrm{NMR}\left(\mathrm{CDCl}_{3}, 300 \mathrm{MHz}\right) \delta 3.83$ (s, 3H), 4.83 (brs, $1 \mathrm{H}), 5.43(\mathrm{~s}, 1 \mathrm{H}), 6.68-6.80(\mathrm{~m}, 4 \mathrm{H}), 7.24-7.30(\mathrm{~m}, 3 \mathrm{H}), 7.41-7.50(\mathrm{~m}, 3 \mathrm{H}), 7.58-7.63(\mathrm{~m}, 2 \mathrm{H}), 7.82(\mathrm{~s}$, 1H). ${ }^{13} \mathrm{C}-\mathrm{NMR}\left(\mathrm{CDCl}_{3}, 75 \mathrm{MHz}\right) \delta 49.9,55.4,85.3,87.9,109.6,111.7,118.0,121.1,122.6,122.8$, 125.9, 128.2, 128.4, 130.3, 130.3, 131.1, 131.8, 136.1, 142.3, 147.2. IR (neat): 3436, 3177, 2965, 2939, 2893, 1600, 1513, 1454, $1374 \mathrm{~cm}^{-1}$. Anal. Calcd. for $\mathrm{C}_{22} \mathrm{H}_{18} \mathrm{BrNO}$ : C, 67.36; H, 4.62; N, 3.57. Found: C, 67.39; H, 4.70; N, 3.53.

N-(2-methoxyphenyl)-3-amino-3-(4-bromophenyl)-1-phenylprop-1-yne (3ra). Prepared starting from aldehyde $7 \mathbf{d}(0.037 \mathrm{~g}, 0.2 \mathrm{mmol})$ and phenylacetylene (2a, $0.051 \mathrm{~g}, 0.5 \mathrm{mmol})$ according to Method A. Purification by flash column chromatography $\left(\mathrm{CH}_{2} \mathrm{Cl}_{2}\right.$ / cyclohexane 2:3) afforded pure 3ra. Yield $0.057 \mathrm{~g}(0.15 \mathrm{mmol}, 73 \%)$; yellow oil. ${ }^{1} \mathrm{H}-\mathrm{NMR}\left(\mathrm{CDCl}_{3}, 400 \mathrm{MHz}\right) \delta 3.83$ (s, 3H), 4.90 (brs, $\left.1 \mathrm{H}\right)$, $5.43(\mathrm{~s}, 1 \mathrm{H}), 6.78-6.85(\mathrm{~m}, 4 \mathrm{H}), 7.26-7.33(\mathrm{~m}, 3 \mathrm{H}), 7.42-7.45(\mathrm{~m}, 2 \mathrm{H}), 7.57-7.63(\mathrm{~m}, 4 \mathrm{H}) .{ }^{13} \mathrm{C}-\mathrm{NMR}$ $\left(\mathrm{CDCl}_{3}, 100 \mathrm{MHz}\right) \delta 49.8,55.4,85.1,88.1,109.8,111.9,118.0,121.3,122.1,122.9,128.5,128.7$, 129.3, 132.0, 132.1, 136.3, 139.3, 147.4. IR (neat): 3430, 3170, 2961, 2928, 2893, 1604, 1510, 1450, $1371 \mathrm{~cm}^{-1}$. Anal. Calcd. for $\mathrm{C}_{22} \mathrm{H}_{18} \mathrm{BrNO}$ : C, 67.36; H, 4.62; N, 3.57. Found: C, 67.38; H, 4.65; N, 3.56.

N-(2-methoxyphenyl)-3-amino-3-(2-naphthyl)-1-phenylprop-1-yne (3sa). Prepared starting from aldehyde $7 \mathbf{e}(0.031 \mathrm{~g}, 0.2 \mathrm{mmol})$ and phenylacetylene (2a, $0.051 \mathrm{~g}, 0.5 \mathrm{mmol})$ according to Method A. Purification by flash column chromatography (diethyl ether / cyclohexane 1:9) afforded pure 3sa. Yield $0.067 \mathrm{~g}(0.18 \mathrm{mmol}, 92 \%)$; yellow oil. ${ }^{1} \mathrm{H}-\mathrm{NMR}\left(\mathrm{CDCl}_{3}, 400 \mathrm{MHz}\right) \delta 3.84$ (s, 3H); 4.92 (brs, $\left.1 \mathrm{H}\right), 5.62$ $(\mathrm{s}, 1 \mathrm{H}), 6.78-6.85(\mathrm{~m}, 4 \mathrm{H}), 7.26-7.33(\mathrm{~m}, 3 \mathrm{H}), 7.42-7.45(\mathrm{~m}, 4 \mathrm{H}), 7.78(\mathrm{~d}, J=7.4 \mathrm{~Hz}, 2 \mathrm{H}), 7.80-7.90$ $(\mathrm{m}, 2 \mathrm{H}), 8.15(\mathrm{~s}, 1 \mathrm{H}) .{ }^{13} \mathrm{C}-\mathrm{NMR}\left(\mathrm{CDCl}_{3}, 100 \mathrm{MHz}\right) \delta 50.6,55.4,85.1,88.7,109.6,111.7,117.8,121.1$, $122.9,125.4,126.1,126.2,126.3,127.7,128.1,128.2,128.2,128.6,131.8,133.1,133.4,136.5,137.3$, 147.2. IR (neat): $3423,3058,2919,2833,1594,1500,1241,1208 \mathrm{~cm}^{-1}$. Anal. Calcd. for $\mathrm{C}_{26} \mathrm{H}_{21} \mathrm{NO}$ : C, 85.92; H, 5.82; N, 3.85. Found: C, 86.03; H, 5.85; N, 3.81 .

N-(2-methoxyphenyl)-3-amino-3-biphenyl-1-phenylprop-1-yne (3ta). Prepared starting from aldehyde $7 \mathbf{f}(0.037 \mathrm{~g}, 0.2 \mathrm{mmol})$ and phenylacetylene (2a, $0.051 \mathrm{~g}, 0.5 \mathrm{mmol})$ according to Method A. Purification by flash column chromatography $\left(\mathrm{CH}_{2} \mathrm{Cl}_{2} /\right.$ cyclohexane 3:7) afforded pure 3ta. Yield 0.23 g (0.06 mmol, 30\%); yellow oil. ${ }^{1} \mathrm{H}-\mathrm{NMR}\left(\mathrm{CDCl}_{3}, 400 \mathrm{MHz}\right) \delta 3.84(\mathrm{~s}, 3 \mathrm{H}) ; 4.81$ (brs, $\left.1 \mathrm{H}\right) ; 5.58(\mathrm{~s}$, $1 \mathrm{H}) ; 6.73-6.77(\mathrm{~m}, 1 \mathrm{H}) ; 6.82(\mathrm{~d}, J=7.2 \mathrm{~Hz}, 1 \mathrm{H}), 6.90-6.92(\mathrm{~m}, 2 \mathrm{H}), 7.28-7.31(\mathrm{~m}, 3 \mathrm{H}), 7.36-7.48(\mathrm{~m}$, 5H), 7.59-7.62 (m, 2H), $7.64(\mathrm{t}, J=8.7 \mathrm{~Hz}, 2 \mathrm{H}), 7.45(\mathrm{~d}, J=8.7 \mathrm{~Hz}, 2 \mathrm{H}) .{ }^{13} \mathrm{C}-\mathrm{NMR}\left(\mathrm{CDCl}_{3}, 100 \mathrm{MHz}\right)$ 
$\delta$ 50.0, 55.4, 84.9, 88.6, 105.5, 109.5, 111.6, 117.6, 121.1, 122.9, 127.1, 127.5, 127.8, 128.1, 128.18, 128.24, 128.8, 131.8, 136.4, 138.9, 140.9, 147.2. IR (neat): 3423, 3051, 2925, 2826, 1593, 1500, 1235, $1020 \mathrm{~cm}^{-1}$. Anal. Calcd. for $\mathrm{C}_{28} \mathrm{H}_{23} \mathrm{NO}$ : C, 86.34; H, 5.95; N, 3.60. Found: C, 86.40; H, 5.98; N, 3.56.

N-(2-methoxyphenyl)-3-amino-3-(3-nitrophenyl)-1-phenylprop-1-yne (3ua). Prepared starting from aldehyde $7 \mathrm{~g}(0.030 \mathrm{~g}, 0.2 \mathrm{mmol})$ and phenylacetylene (2a, $0.051 \mathrm{~g}, 0.5 \mathrm{mmol})$ according to Method A. Purification by flash column chromatography $\left(\mathrm{CH}_{2} \mathrm{Cl}_{2} /\right.$ cyclohexane 3:7) afforded pure 3ua. Yield $0.047 \mathrm{~g}(0.13 \mathrm{mmol}, 66 \%)$; yellow oil. ${ }^{1} \mathrm{H}-\mathrm{NMR}\left(\mathrm{CDCl}_{3}, 300 \mathrm{MHz}\right) \delta 3.85$ (s, 3H); 5.10 (brs, $\left.1 \mathrm{H}\right), 5.60$ $(\mathrm{s}, 1 \mathrm{H}), 6.67-6.86(\mathrm{~m}, 4 \mathrm{H}), 7.26-7.33(\mathrm{~m}, 3 \mathrm{H}), 7.42-7.45(\mathrm{~m}, 2 \mathrm{H}), 7.57(\mathrm{t}, J=8.1 \mathrm{~Hz}, 1 \mathrm{H}), 8.01(\mathrm{~d}, J=$ $7.8 \mathrm{~Hz}, 1 \mathrm{H}), 8.20(\mathrm{dd}, J=8.1 \mathrm{~Hz}, J=0.9 \mathrm{~Hz}, 1 \mathrm{H}), 8.48(\mathrm{~s}, 1 \mathrm{H}) .{ }^{13} \mathrm{C}-\mathrm{NMR}\left(\mathrm{CDCl}_{3}, 75 \mathrm{MHz}\right) \delta 49.9$, 55.4, 85.9, 87.2, 109.8, 111.7, 118.5, 121.0, 122.3, 123.0, 128.3, 128.6, 129.6, 131.8, 133.3, 135.7, 142.4, 147.3, 146.6. IR (neat): 3423, 3051, 2925, 2826, 1593, 1500, 1235, $1020 \mathrm{~cm}^{-1}$. Anal. Calcd. for $\mathrm{C}_{22} \mathrm{H}_{18} \mathrm{~N}_{2} \mathrm{O}_{3}$ : C, 73.73; H, 5.06; N, 7.82. Found: C, 73.80; H, 5.13; N, 7.75.

\section{N-(2-methoxyphenyl)-3-amino-3-(4-trifluoromethylphenyl)-1-phenylprop-1-yne (3va). Prepared} starting from aldehyde $7 \mathbf{h}(0.035 \mathrm{~g}, 0.2 \mathrm{mmol})$ and phenylacetylene (2a, $0.051 \mathrm{~g}, 0.5 \mathrm{mmol})$ according to Method A. Purification by flash column chromatography (diethyl ether / cyclohexane 1:9) afforded pure 3va. Yield $0.060 \mathrm{~g}(0.16 \mathrm{mmol}, 79 \%)$; yellow oil. ${ }^{1} \mathrm{H}-\mathrm{NMR}\left(\mathrm{CDCl}_{3}, 300 \mathrm{MHz}\right) \delta 3.84(\mathrm{~s}, 3 \mathrm{H}), 5.58$ $(\mathrm{s}, 1 \mathrm{H}), 6.68-6.85(\mathrm{~m}, 4 \mathrm{H})$; 7.27-7.35 (m, 3H), 7.38-7.44 (m, 2H), 7.65 (d, $J=8.4 \mathrm{~Hz}, 2 \mathrm{H}), 7.79(\mathrm{~d}, J=$ $8.4 \mathrm{~Hz}, 2 \mathrm{H}) .{ }^{13} \mathrm{C}-\mathrm{NMR}\left(\mathrm{CDCl}_{3}, 75 \mathrm{MHz}\right) \delta$ 50.0, 55.4, 85.4, 87.7, 109.7, 111.7, 118.2, 120.1, 121.1, $122.5,125.67,125.72,127.6,128.3,128.5,131.8,136.0,144.0,147.2$. IR (neat): 3390, 3270, 2919, 2853, 1475, 1380, $\mathrm{cm}^{-1}$. Anal. Calcd. for $\mathrm{C}_{23} \mathrm{H}_{18} \mathrm{~F}_{3} \mathrm{NO}$ : C, 72.43; H, 4.76; N, 3.67. Found: C, 72.48; H, $4.79 ; \mathrm{N}, 3.62$.

4-[1-(2-Methoxy-3-phenylamino)-3-phenylpropyn-2-yl]-benzoic acid methyl ester (3wa). Prepared starting from aldehyde $7 \mathbf{i}(0.033 \mathrm{~g}, 0.2 \mathrm{mmol})$ and phenylacetylene $(2 \mathrm{a}, 0.050 \mathrm{~g}, 0.5 \mathrm{mmol})$ according to Method A. Purification by flash column chromatography (diethyl ether / cyclohexane 1:4) afforded pure 3wa. Yield $0.048 \mathrm{~g}(0.13 \mathrm{mmol}, 65 \%)$; yellow oil. ${ }^{1} \mathrm{H}-\mathrm{NMR}\left(\mathrm{CDCl}_{3}, 300 \mathrm{MHz}\right) \delta 3.81(\mathrm{~s}, 3 \mathrm{H}), 3.88(\mathrm{~s}$, $3 \mathrm{H}), 5.50(\mathrm{~s}, 1 \mathrm{H}), 6.69-6.84(\mathrm{~m}, 4 \mathrm{H}), 7.22-7.28(\mathrm{~m}, 3 \mathrm{H}), 7.36-7.40(\mathrm{~m}, 2 \mathrm{H}), 7.70(\mathrm{~d}, J=8.4 \mathrm{~Hz}, 2 \mathrm{H})$, $8.04(\mathrm{~d}, J=8.4 \mathrm{~Hz}, 2 \mathrm{H}) .{ }^{13} \mathrm{C}-\mathrm{NMR}\left(\mathrm{CDCl}_{3}, 75 \mathrm{MHz}\right) \delta 50.1,52.1,55.4,85.3,87.9,109.6,111.7,118.0$, 121.0, 122.5, 127.2, 128.2, 128.4, 129.8, 130.0, 131.7, 136.0, 145.0, 147.2, 166.7. IR (neat): 3390, 3270, 
2919, 2853, 1475, $1380 \mathrm{~cm}^{-1}$. Anal. Calcd. for $\mathrm{C}_{24} \mathrm{H}_{21} \mathrm{NO}_{3}$ : C, 77.71.; H, 5.70; N, 3.77. Found: C, 77.76; H, 5.78; N, 3.71 .

N-(2-methoxyphenyl)-3-amino-3-(pentafluorophenyl)-1-phenylprop-1-yne (3xa). Prepared starting from aldehyde $7 \mathbf{j}(0.055 \mathrm{~g}, 0.2 \mathrm{mmol})$ and phenylacetylene (2a, $0.051 \mathrm{~g}, 0.5 \mathrm{mmol})$ according to Method A. Purification by flash column chromatography $\left(\mathrm{CH}_{2} \mathrm{Cl}_{2} /\right.$ cyclohexane 35:65) afforded pure 3xa. Yield $0.051 \mathrm{~g}(0.13 \mathrm{mmol}, 63 \%)$; yellow oil. ${ }^{1} \mathrm{H}-\mathrm{NMR}\left(\mathrm{CDCl}_{3}, 400 \mathrm{MHz}\right) \delta 3.84(\mathrm{~s}, 3 \mathrm{H}), 5.95$ (s, $1 \mathrm{H}), 6.72-6.82(\mathrm{~m}, 3 \mathrm{H}), 6.87(\mathrm{dt}, J=7.6 \mathrm{~Hz}, J=1.6 \mathrm{~Hz}, 1 \mathrm{H}), 7.27-7.34(\mathrm{~m}, 3 \mathrm{H}), 7.43-7.46(\mathrm{~m}, 2 \mathrm{H})$. ${ }^{13} \mathrm{C}-\mathrm{NMR}\left(\mathrm{CDCl}_{3}, 100 \mathrm{MHz}\right) \delta$ 40.4, 55.6, 84.4, 85.1, 110.2, 111.2, $114.5(\mathrm{t}, \mathrm{J}=56 \mathrm{~Hz}), 119.0,121.2$, 122.0, 128.3, 128.8, 131.9, 134.8, 136.5 (dt, $J=73 \mathrm{~Hz}, J=24 \mathrm{~Hz}), 139.9$ (dt, $J=66 \mathrm{~Hz}, J=20 \mathrm{~Hz}$ ), 139.0 (m), 143.7 (m), 146.1 (m), 147.7. IR (neat): 3390, 3270, 2919, 2853, 1475, $1380 \mathrm{~cm}^{-1}$. Anal. Calcd. for $\mathrm{C}_{22} \mathrm{H}_{14} \mathrm{~F}_{5} \mathrm{NO}$ : C, 65.51; H, 3.50; N, 3.47. Found: C, 65.48; H, 3.41; N, 3.49.

N-(2-methoxyphenyl)-3-amino-1-phenyl-3-(3-pyridyl)-prop-1-yne (3ya). Prepared starting from aldehyde 7k (0.021 g, $0.2 \mathrm{mmol})$ and phenylacetylene (2a, $0.051 \mathrm{~g}, 0.5 \mathrm{mmol})$ according to Method A. Purification by flash column chromatography (ethyl acetate / cyclohexane 55:45) afforded pure 3ya. Yield $0.041 \mathrm{~g}$ (0.13 mmol, 65\%); yellow oil. ${ }^{1} \mathrm{H}-\mathrm{NMR}\left(\mathrm{CDCl}_{3}, 300 \mathrm{MHz}\right) \delta 3.84(\mathrm{~s}, 3 \mathrm{H}), 5.60(\mathrm{~s}, 1 \mathrm{H})$, 6.78-6.93 (m, 4H), 7.30-7.34 (m, 3H), 7.42-7.46 (m, 3H), $8.09(\mathrm{~d}, J=7.5 \mathrm{~Hz}, 1 \mathrm{H}), 8.64(\mathrm{~d}, J=5.1 \mathrm{~Hz}$, $1 \mathrm{H}), 8.97(\mathrm{~s}, 1 \mathrm{H}) .{ }^{13} \mathrm{C}-\mathrm{NMR}\left(\mathrm{CDCl}_{3}, 75 \mathrm{MHz}\right) \delta 48.2,55.4,85.6,87.3,109.7,111.8,118.3,121.0$, 122.4, 123.6, 128.2, 128.5, 131.7, 135.0, 135.7, 135.9, 147.3, 148.9, 149.1. IR (neat): 3390, 3270, 2919, 2853, 1475, 1380, $\mathrm{cm}^{-1}$. Anal. Calcd. for $\mathrm{C}_{21} \mathrm{H}_{18} \mathrm{~N}_{2} \mathrm{O}$ : C, 80.23; H, 5.77; N, 8.91. Found: C, 80.20; $\mathrm{H}$, $5.79 ; \mathrm{N}, 8.93$.

N-(2-methoxyphenyl)-3-amino-3-cyclohexyl-1-phenylprop-1-yne (3za). Prepared starting from aldehyde $7 \mathrm{l}$ (0.044 g, $0.4 \mathrm{mmol})$ and phenylacetylene (2a, $0.102 \mathrm{~g}, 1.0 \mathrm{mmol})$ according to Method B. Purification by flash column chromatography $\left(\mathrm{CH}_{2} \mathrm{Cl}_{2} /\right.$ cyclohexane 15:85) afforded pure 3za. Yield $0.109 \mathrm{~g}(0.34 \mathrm{mmol}, 85 \%)$; yellow oil. ${ }^{1} \mathrm{H}-\mathrm{NMR}\left(\mathrm{CDCl}_{3}, 300 \mathrm{MHz}\right) \delta 1.35-1.47$ (m, 6H), 1.74-2.08 $(\mathrm{m}, 5 \mathrm{H}), 3.90(\mathrm{~s}, 3 \mathrm{H}), 4.22(\mathrm{~d}, J=5.7 \mathrm{~Hz}, 1 \mathrm{H}), 4.40$ (brs, 1H), 6.62-6.97 (m, 4H), 7.29-7.32 (m, 3H), 7.39-7.44 (m, 2H). ${ }^{13} \mathrm{C}-\mathrm{NMR}\left(\mathrm{CDCl}_{3}, 75 \mathrm{MHz}\right) \delta 26.0,26.1,26.4,42.3,51.3,55.3,83.6,89.1,109.4$, 110.1, 116.9, 121.1, 123.2, 127.8, 128.1, 131.6, 136.8, 147.0 .Anal. Calcd. for $\mathrm{C}_{22} \mathrm{H}_{25} \mathrm{NO}$ : C, 82.72; $\mathrm{H}$, 7.89; N, 4.38. Found: C, 82.70; H, 7.84; N, 4.40 . 
N-(2-methoxyphenyl)-3-amino-4,4-dimethyl-1-phenylpent-1-yne (3Aa). Prepared starting from aldehyde 7m (0.034 g, $0.4 \mathrm{mmol})$ and phenylacetylene (2a, $0.102 \mathrm{~g}, 1.0 \mathrm{mmol})$ according to Method B. Purification by flash column chromatography $\left(\mathrm{CH}_{2} \mathrm{Cl}_{2} /\right.$ cyclohexane 1:4) afforded pure 3Aa. Yield 0.079 g (0.27 mmol, 67\%); yellow oil. ${ }^{1} \mathrm{H}-\mathrm{NMR}\left(\mathrm{CDCl}_{3}, 300 \mathrm{MHz}\right) \delta 1.22(\mathrm{~s}, 9 \mathrm{H}), 3.91(\mathrm{~s}, 3 \mathrm{H}), 4.08(\mathrm{~s}$, 1H), 4.55 (brs, 1H), 6.72-6.78 (m, 1H), 6.83-6.90 (m, 2H), 6.89-6.95 (m, 1H), 7.29-7.38 (m, 3H), 7.38$7.43(\mathrm{~m}, 2 \mathrm{H}) .{ }^{13} \mathrm{C}-\mathrm{NMR}\left(\mathrm{CDCl}_{3}, 75 \mathrm{MHz}\right) \delta$ 26.5, 35.7, 55.4, 55.9, 83.3, 89.4, 109.6, 111.0, 116.9,121.1, 123.3, 127.8, 128.1, 131.6, 137.3, 137.1. Anal. Calcd. for $\mathrm{C}_{20} \mathrm{H}_{23} \mathrm{NO}$ : C, 81.87; H, 7.90; N, 4.77. Found: C, 81.84; H, 7.88; N, 4.80.

N-(2-methoxyphenyl)-3-amino-1-phenyldec-1-yne (3Ba). Prepared starting from aldehyde 7n (0.052 g, $0.4 \mathrm{mmol}$ ) and phenylacetylene (2a, $0.102 \mathrm{~g}, 1.0 \mathrm{mmol})$ according to Method B. Purification by flash column chromatography $\left(\mathrm{CH}_{2} \mathrm{Cl}_{2} /\right.$ cyclohexane 1:4) afforded pure 3Ba. Yield $0.060 \mathrm{~g}(0.18 \mathrm{mmol}$, 45\%); yellow oil. ${ }^{1} \mathrm{H}-\mathrm{NMR}\left(\mathrm{CDCl}_{3}, 300 \mathrm{MHz}\right) \delta$ 0.82-1.00 (m, 3H), 1-20-1.42 (m, 9H), 1.60-1.80 (m, 2H), 1.89-2.02 (m, 1H), $3.90(\mathrm{~s}, 3 \mathrm{H}), 4.34$ (t, $J=6.3 \mathrm{~Hz}, 1 \mathrm{H}), 4.46$ (brs, 1H), 6.73-6.98 (m, 4H), 7.29$7.31(\mathrm{~m}, 3 \mathrm{H}), 7.39-7.43(\mathrm{~m}, 2 \mathrm{H}) .{ }^{13} \mathrm{C}-\mathrm{NMR}\left(\mathrm{CDCl}_{3}, 75 \mathrm{MHz}\right) \delta 14.1,22.6,26.1,29.2,29.2,31.8,35.9$, 45.9, 55.3, 82.6, 90.3, 109.4, 111.3, 117.2, 121.1, 123.2, 127.9, 128.1, 131.7, 136.6, 147.0. Anal. Calcd. for $\mathrm{C}_{23} \mathrm{H}_{29} \mathrm{NO}: \mathrm{C}, 82.34 ; \mathrm{H}, 8.71 ; \mathrm{N}, 4.18$. Found: $\mathrm{C}, 82.31 ; \mathrm{H}, 8.69 ; \mathrm{N}, 4.20$.

\section{Copies of the NMR spectra of compounds 3aa-3na and 3pa-3Ba}




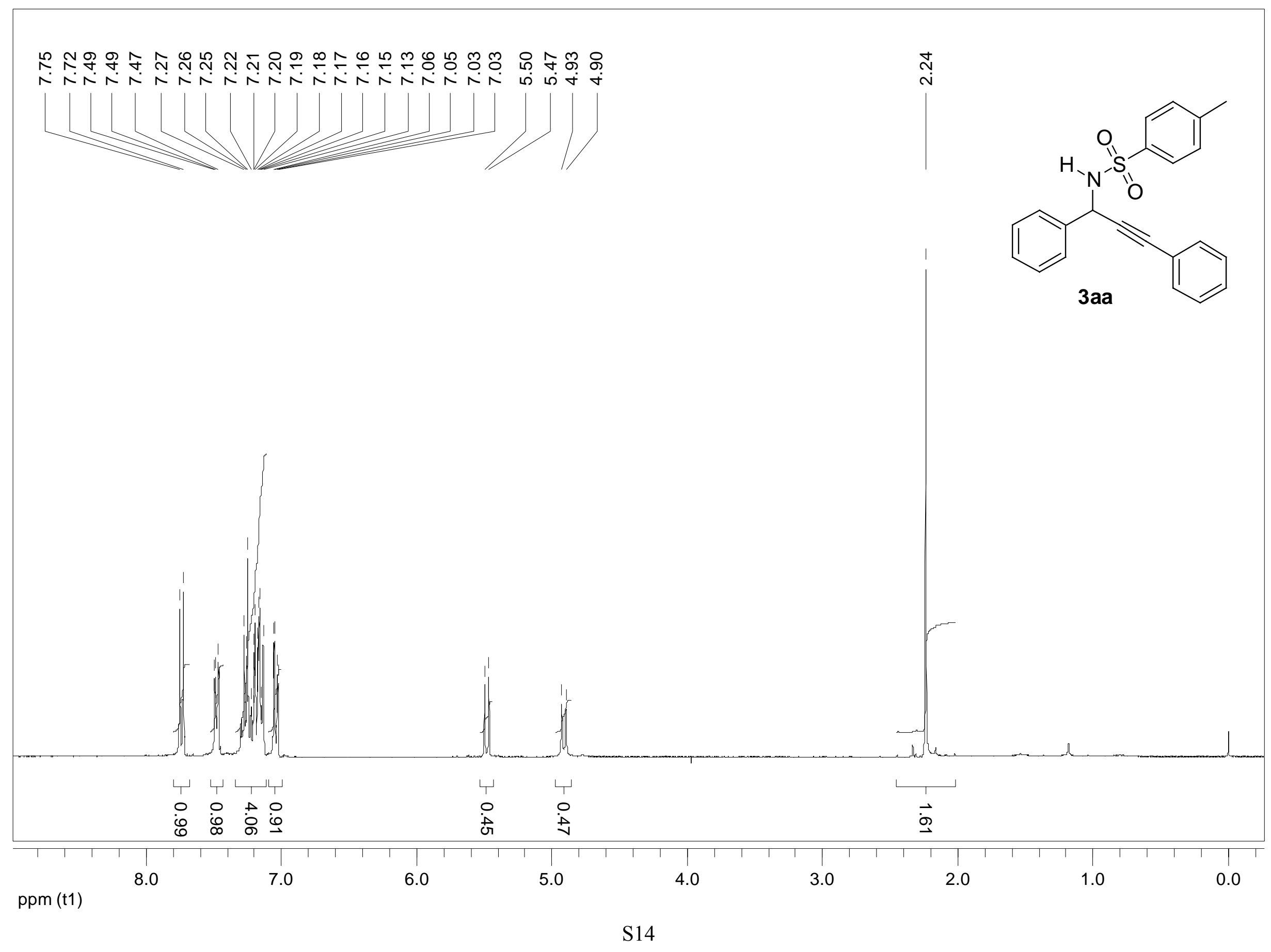




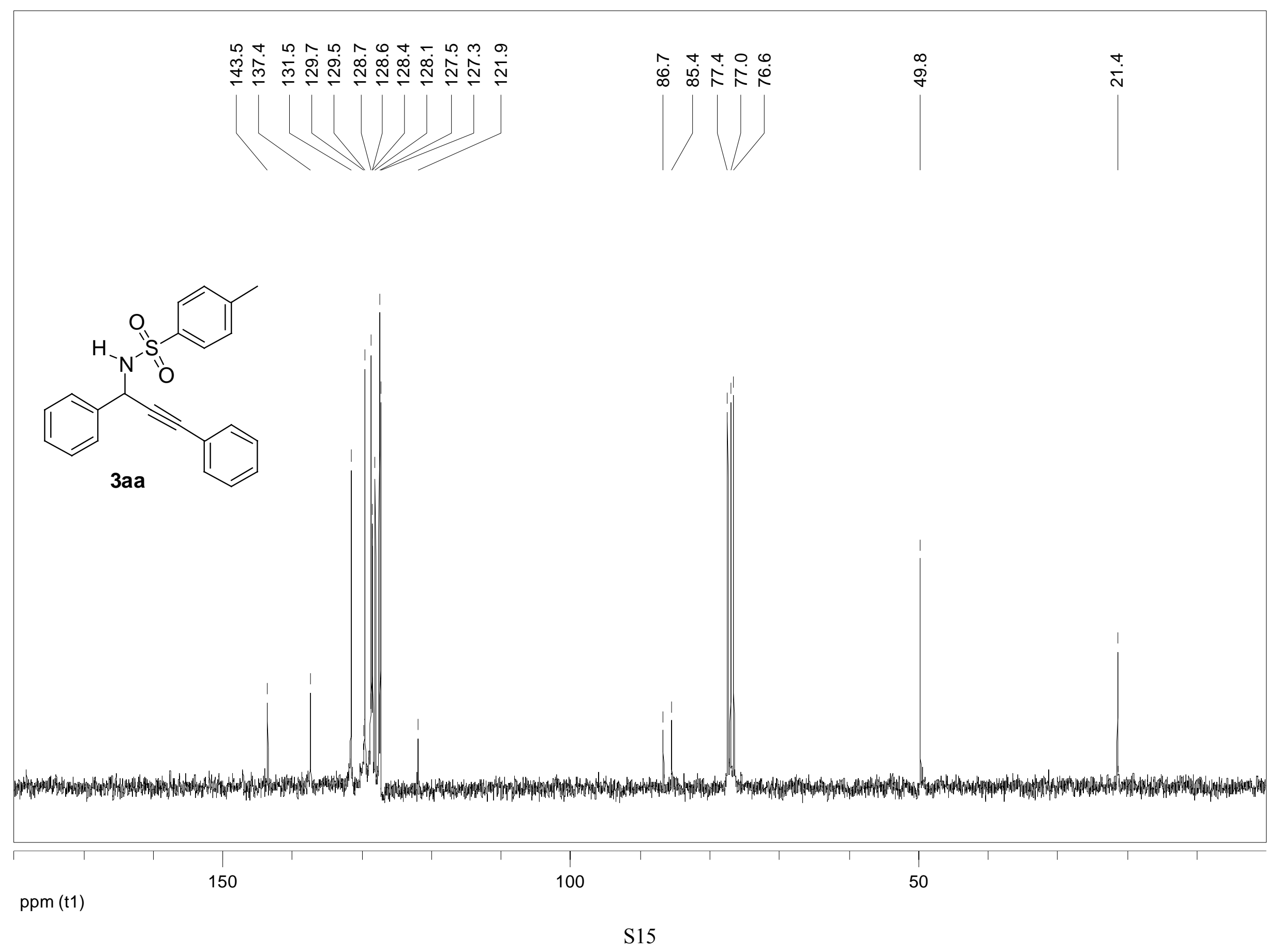




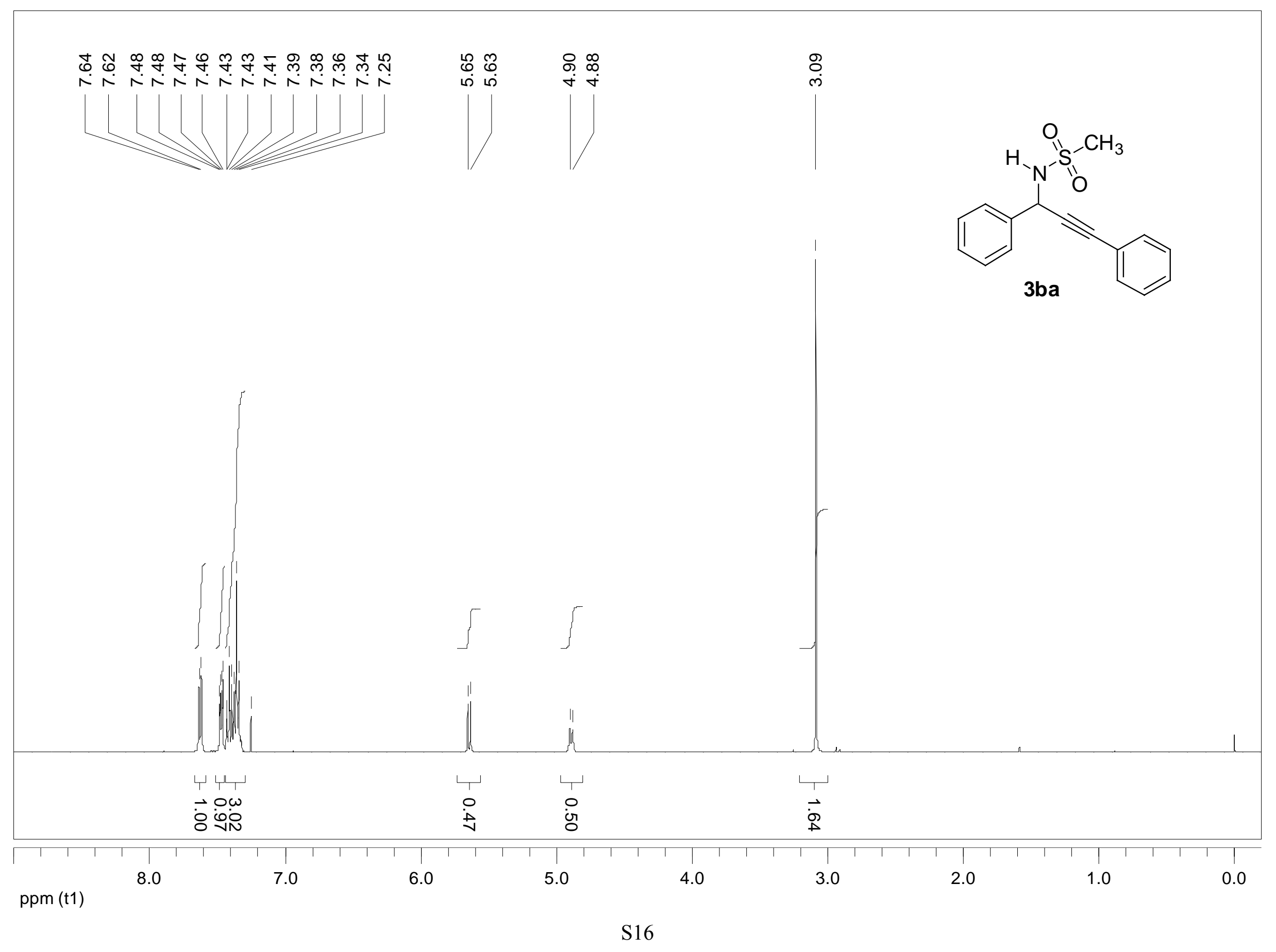




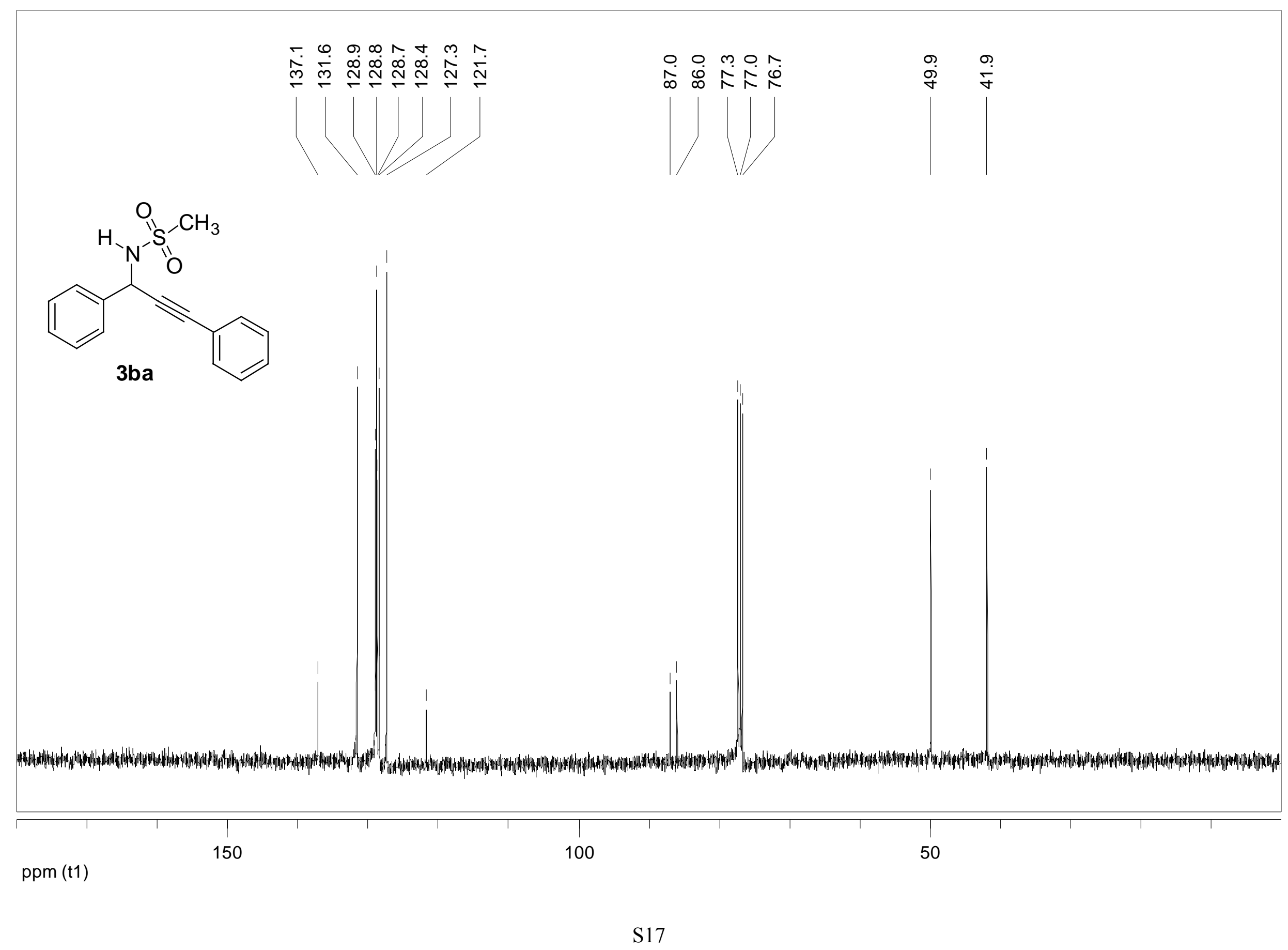




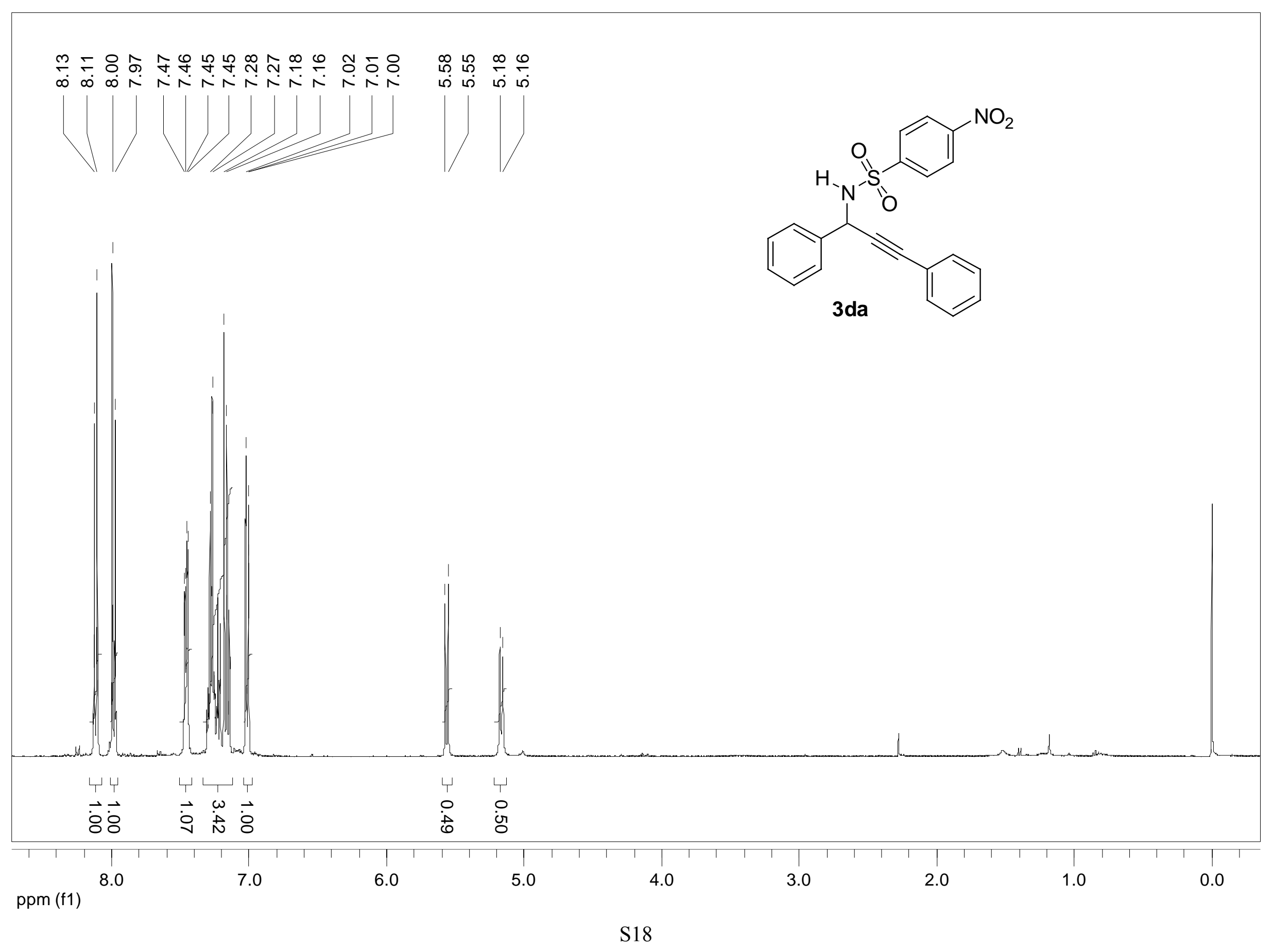




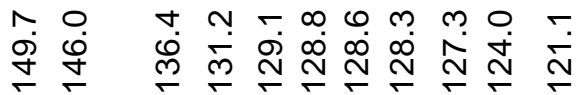

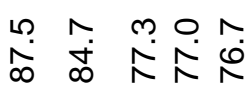

ัก
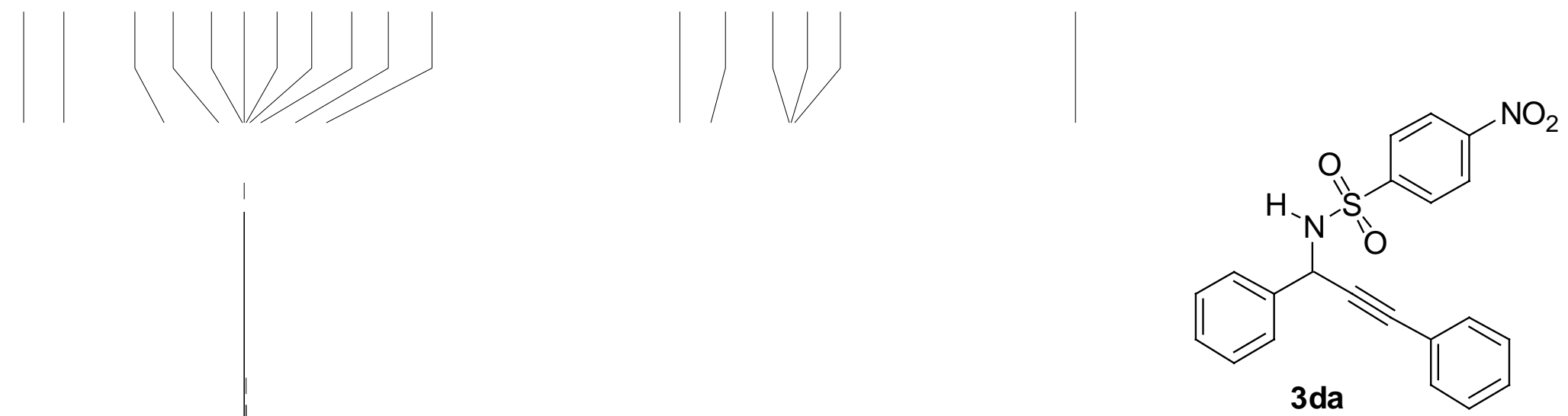

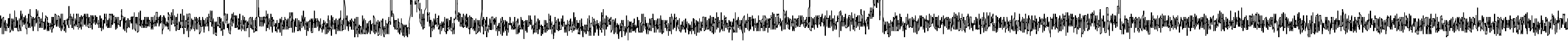

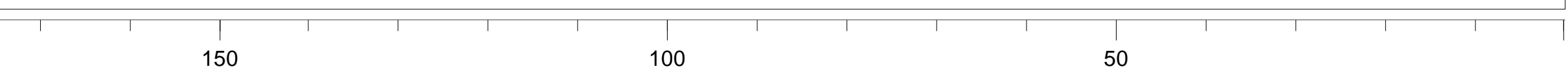

ppm (t1) 


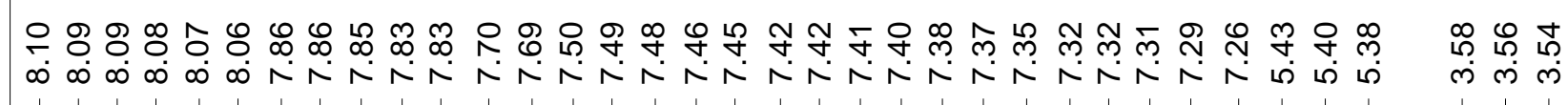

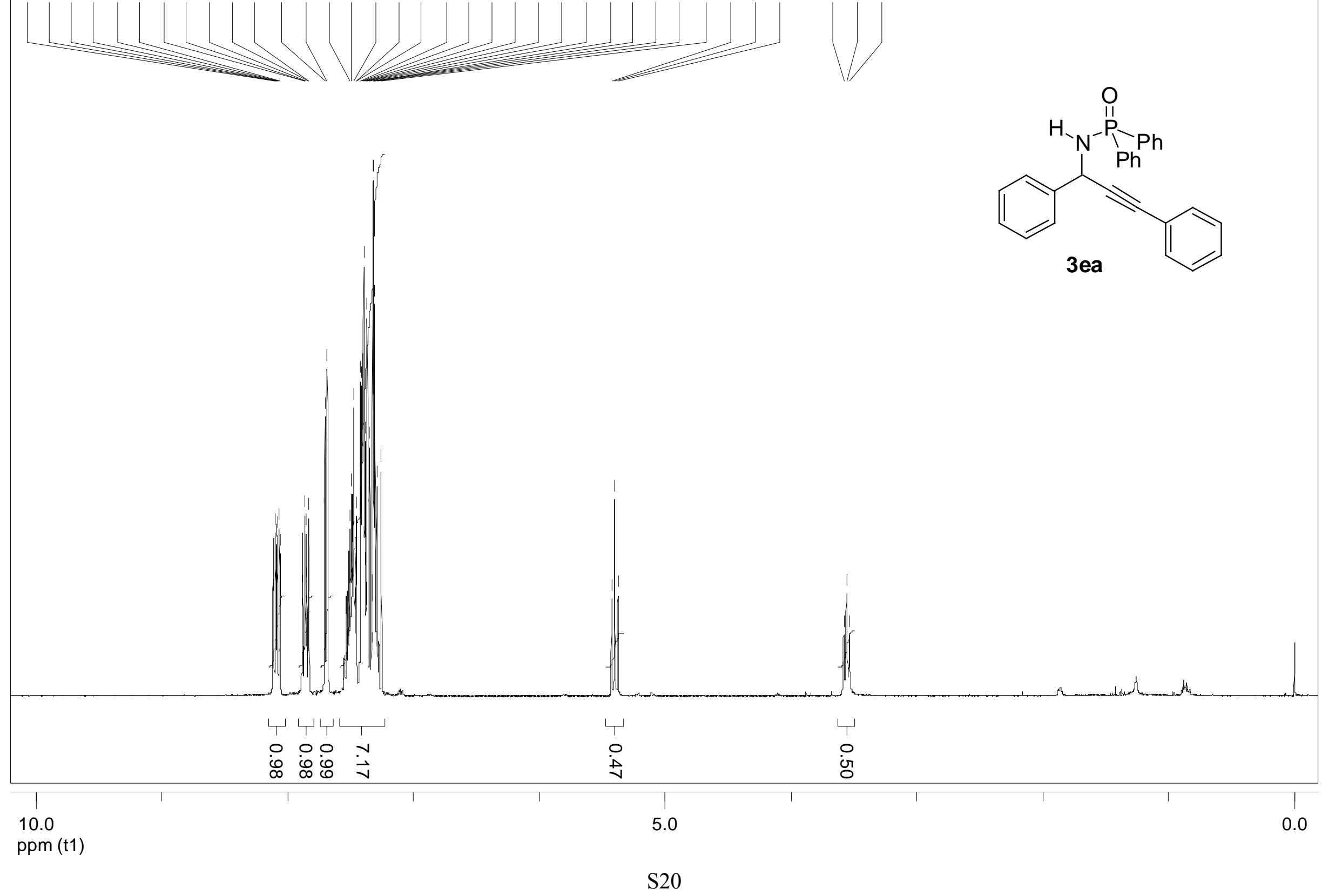




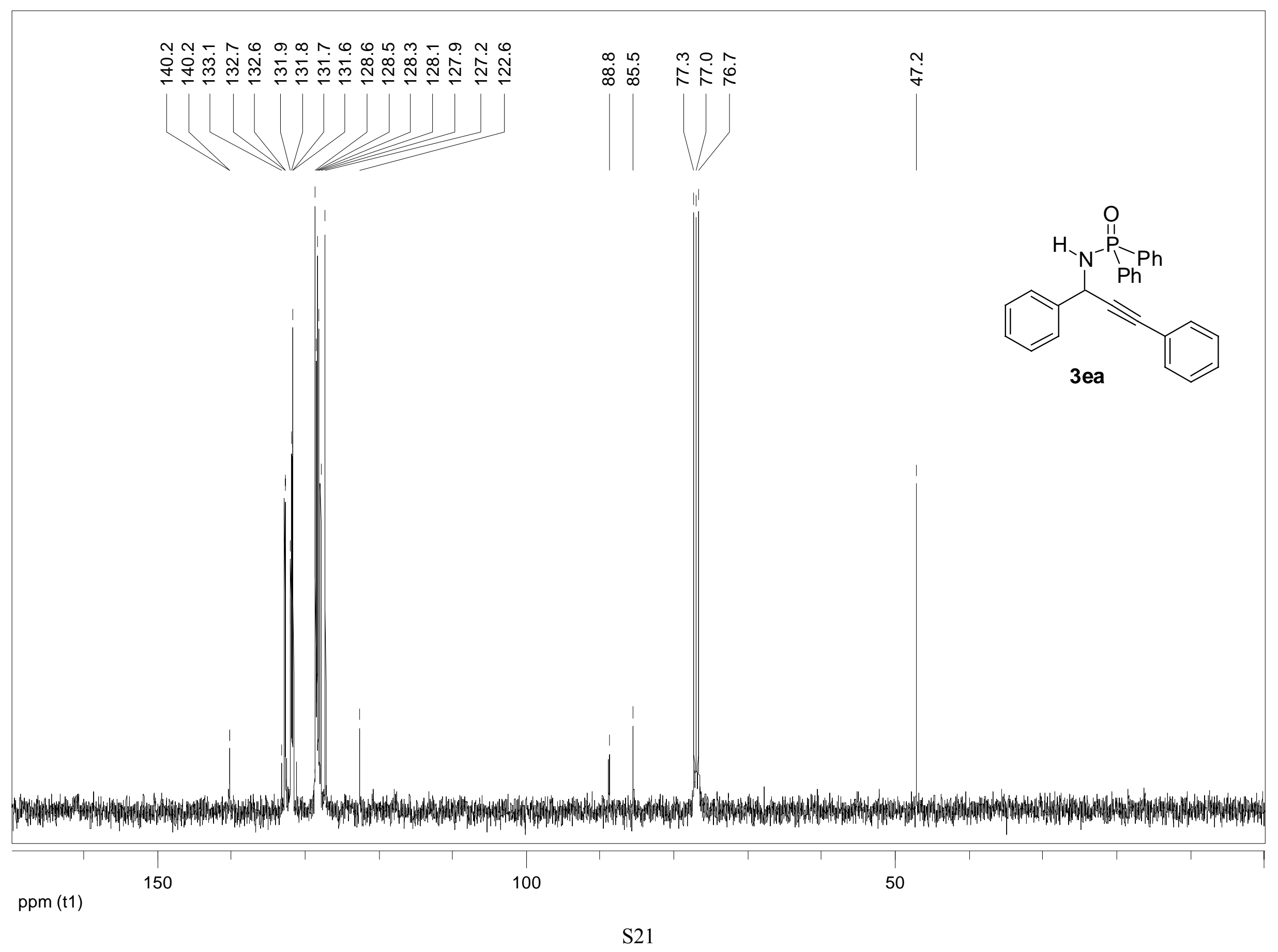


œ

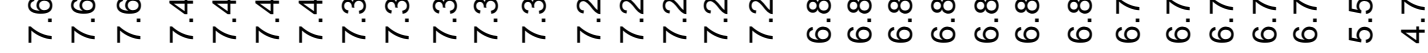

$m$
$\infty$
$\infty$

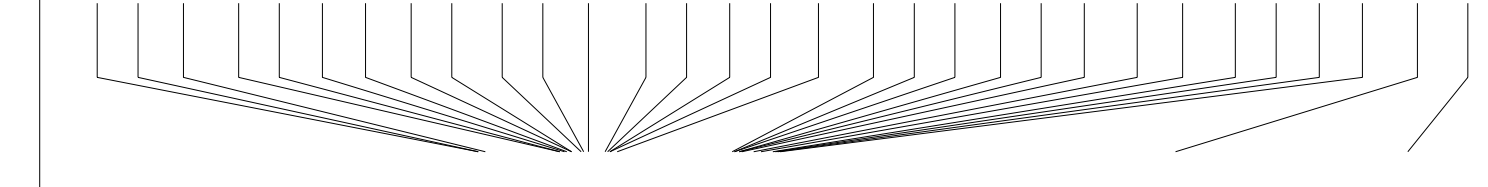

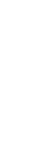
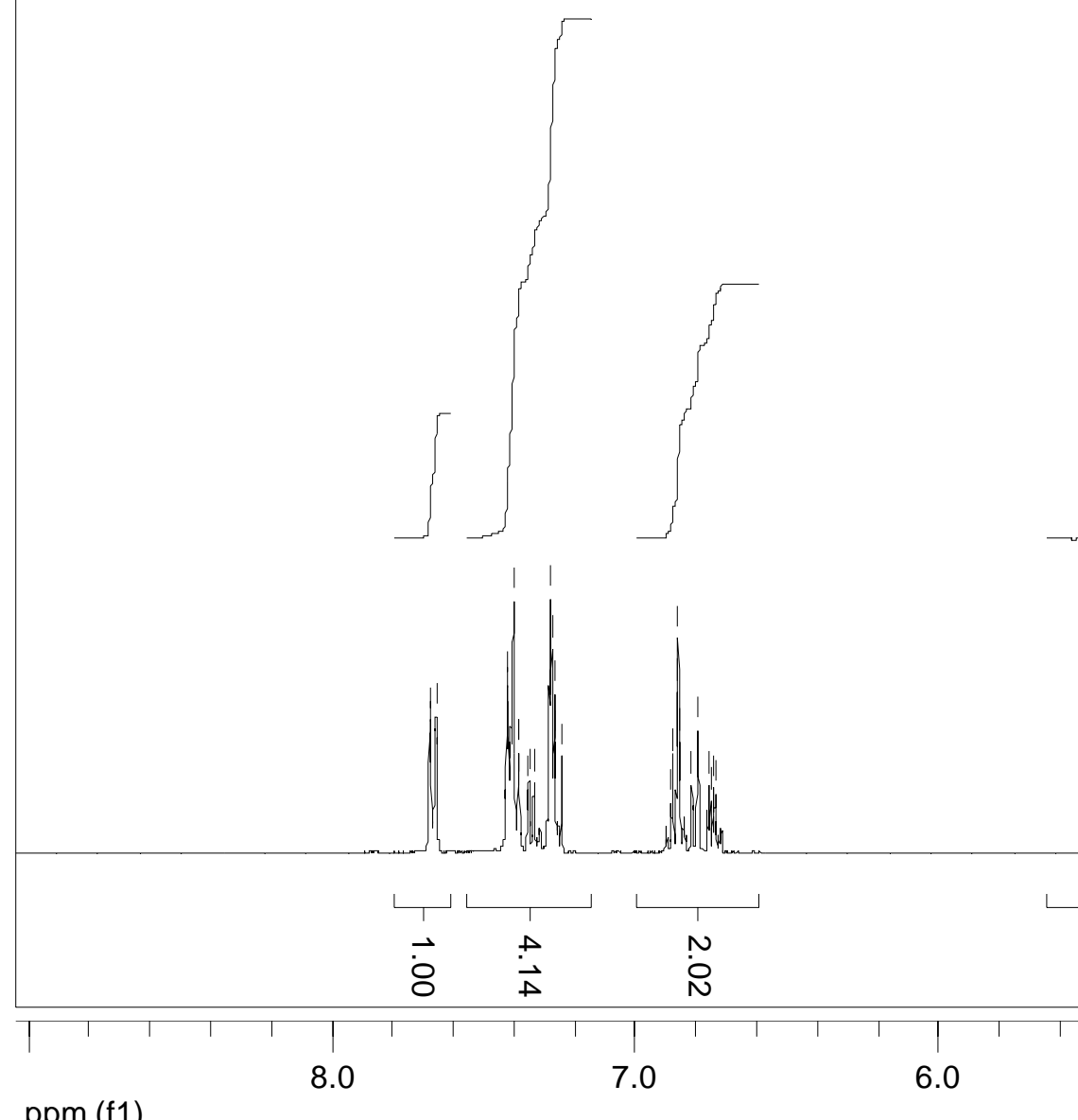

ppm (f1) 


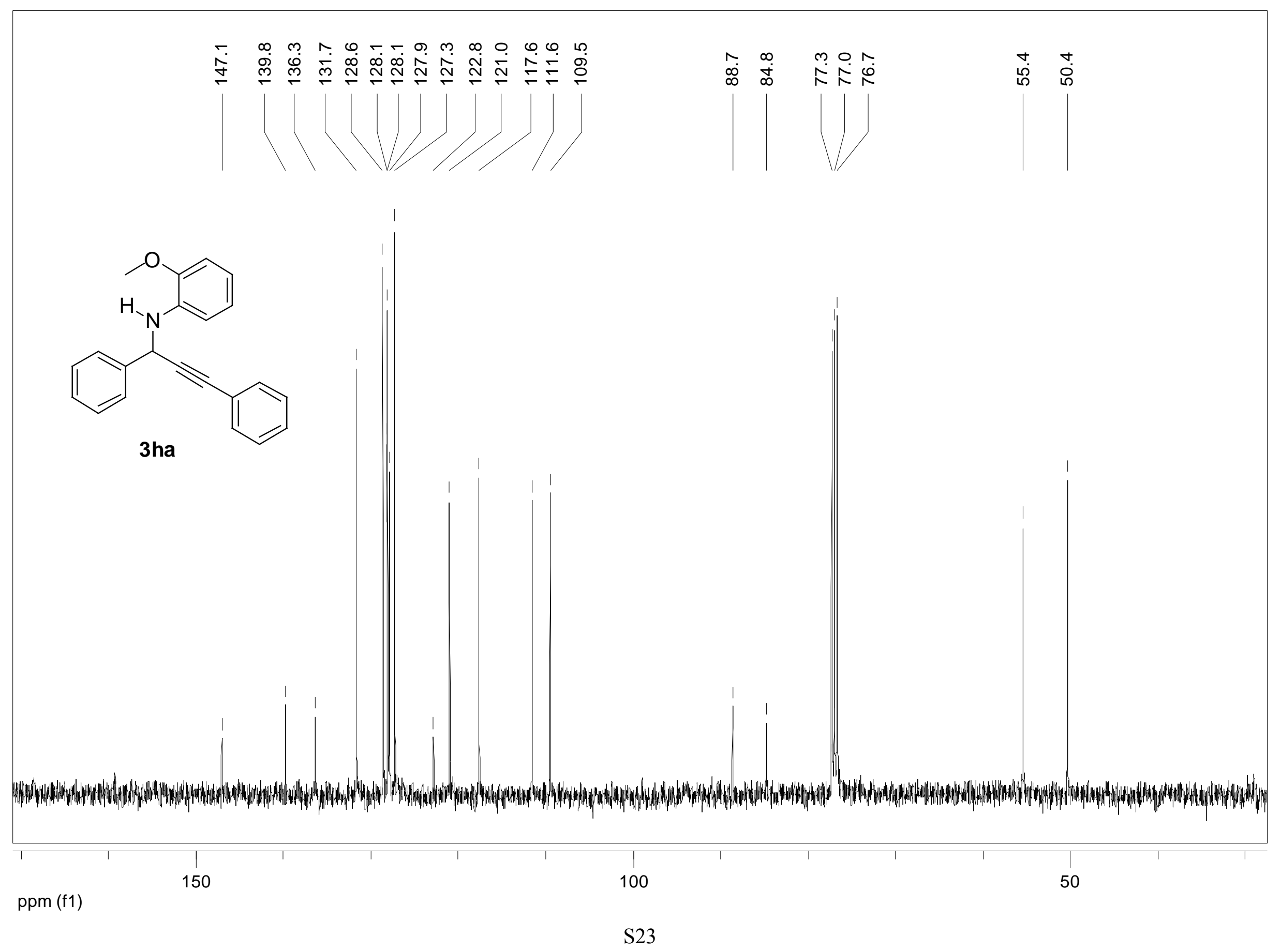




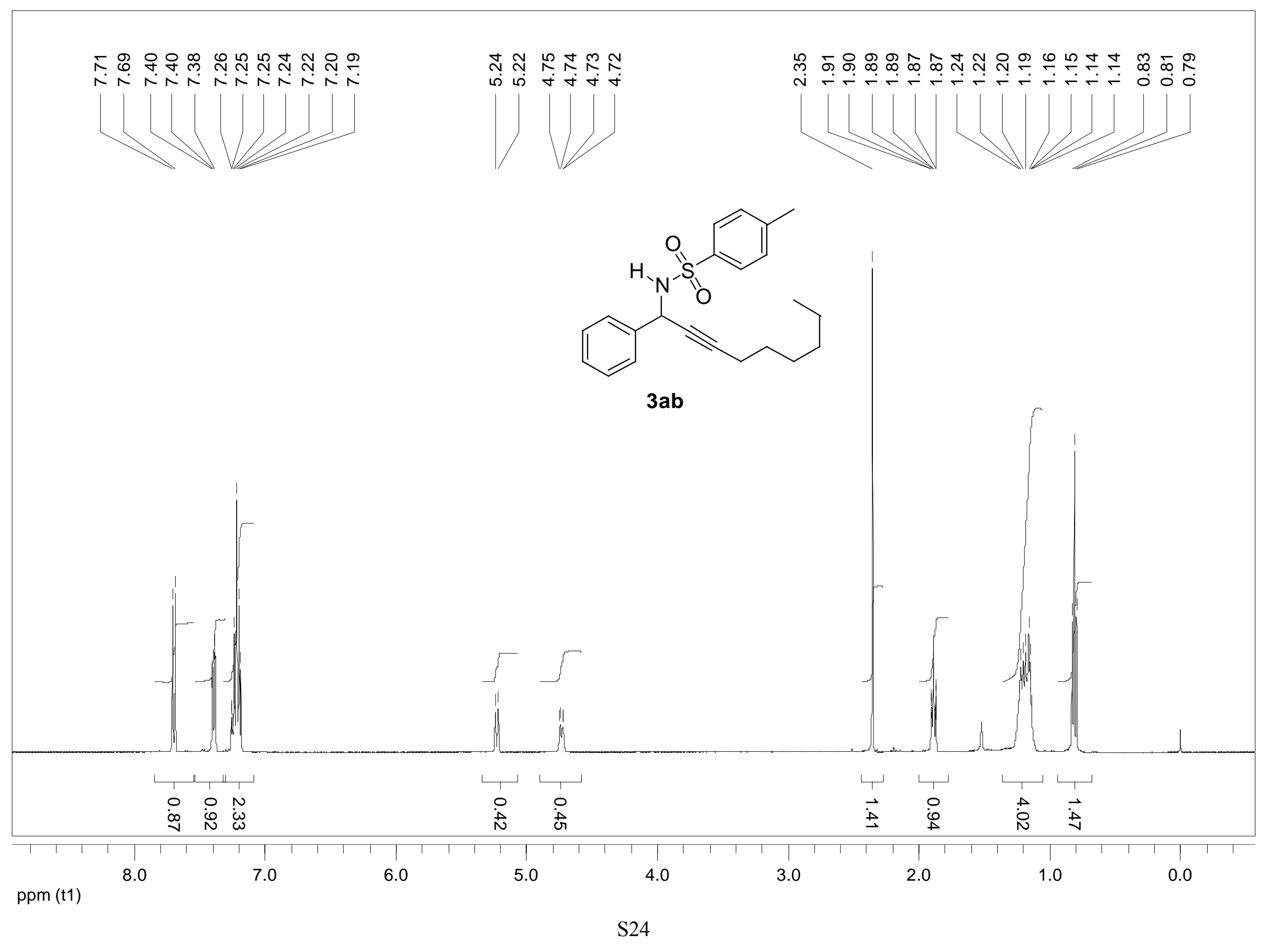




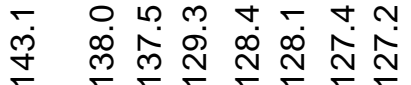

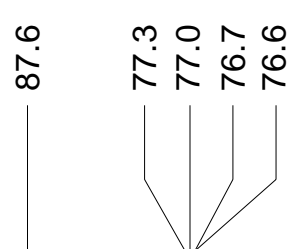

煦

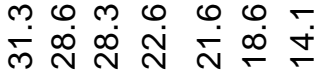
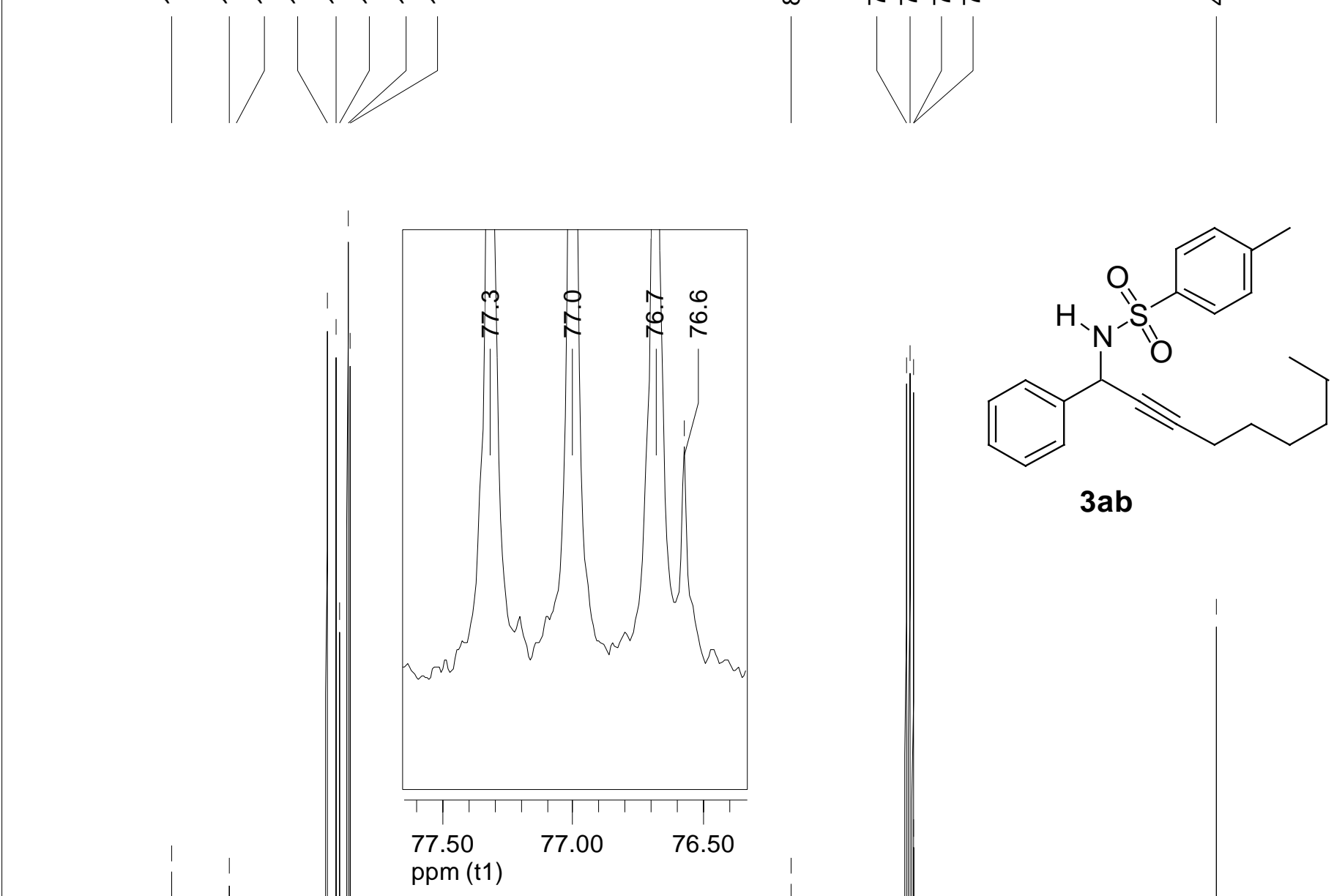

$3 a b$

BAt 


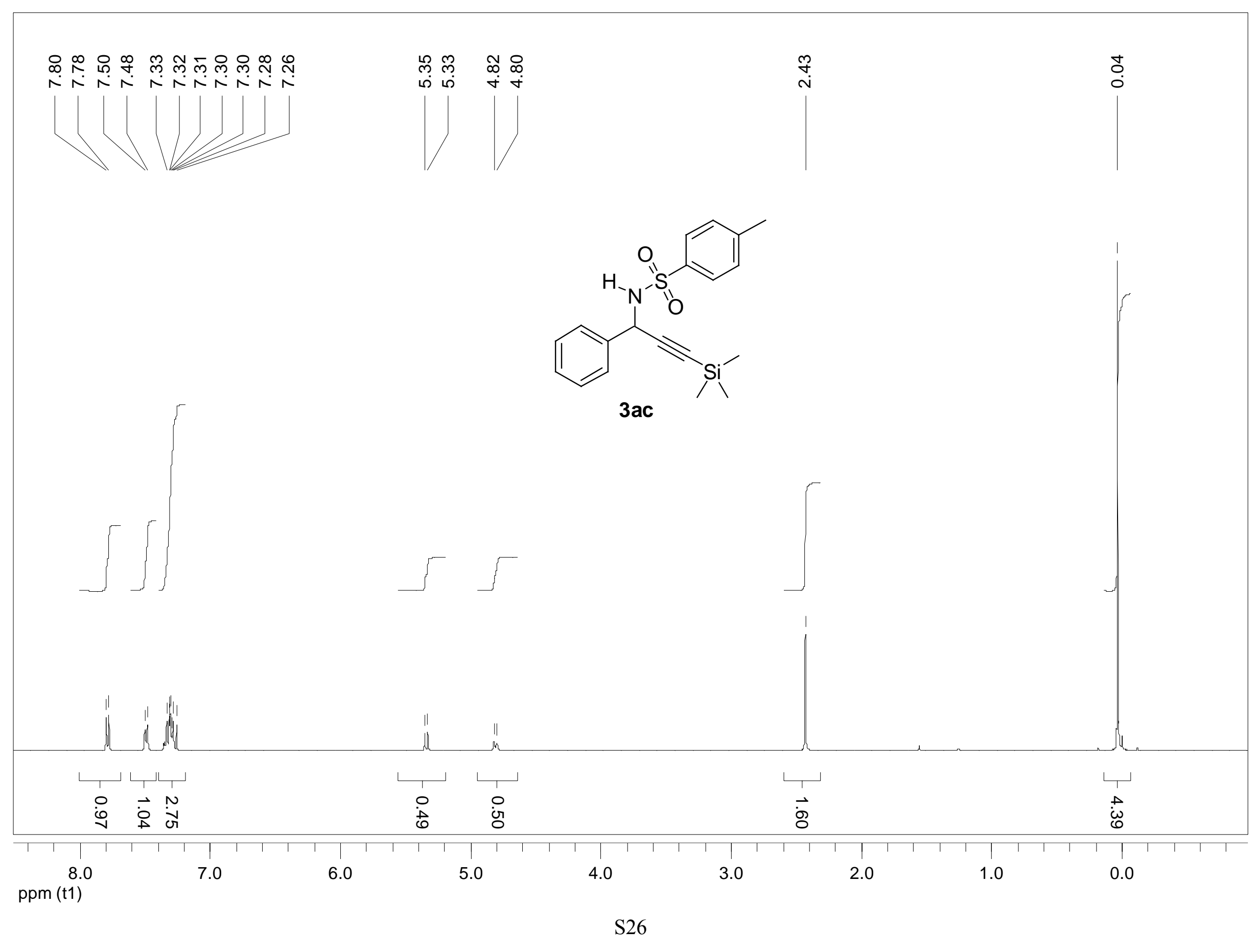




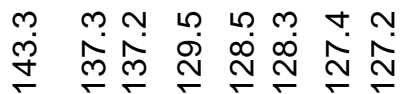

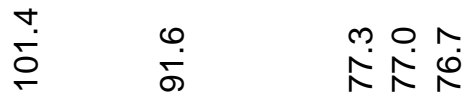

$\hat{\dot{g}}$

$\stackrel{0}{i}$

$\stackrel{m}{\circ}$
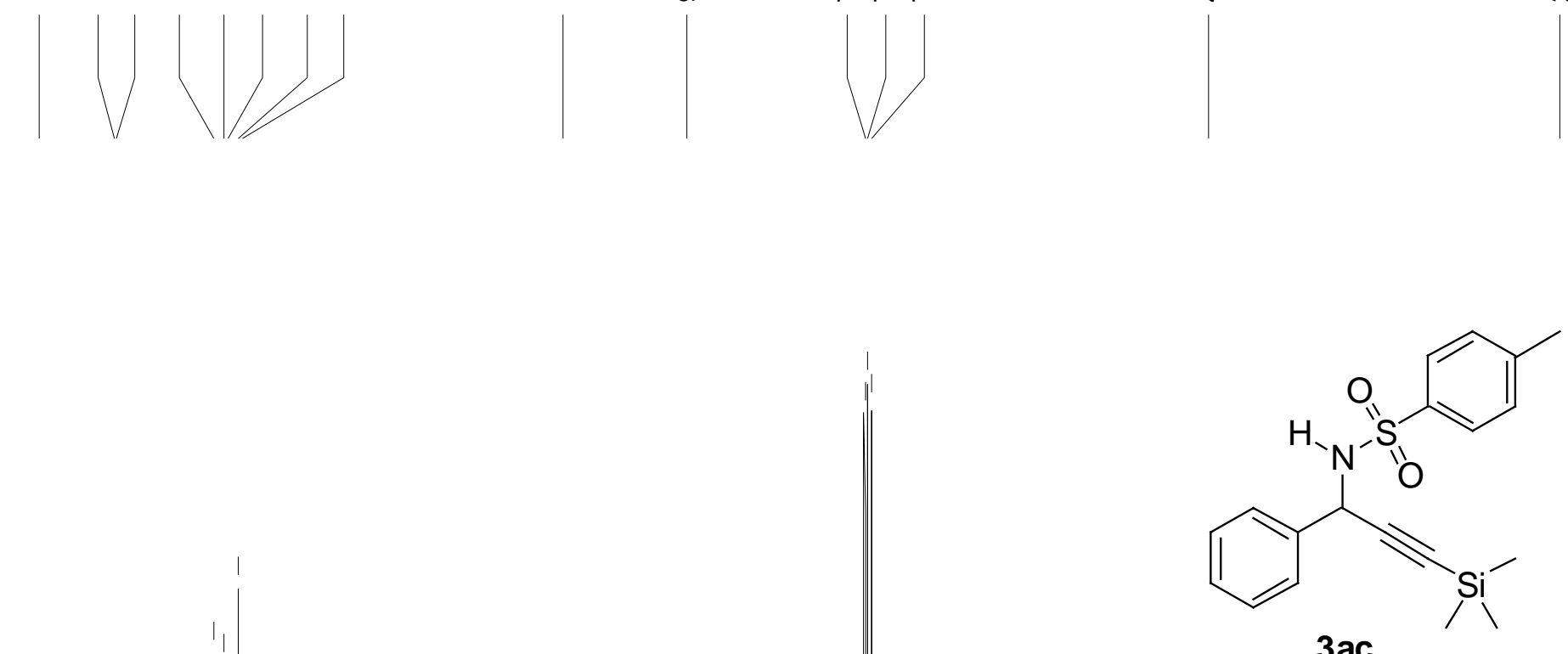

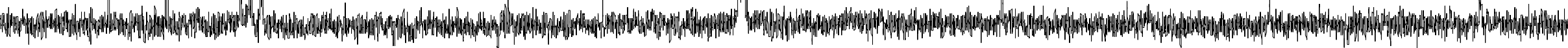

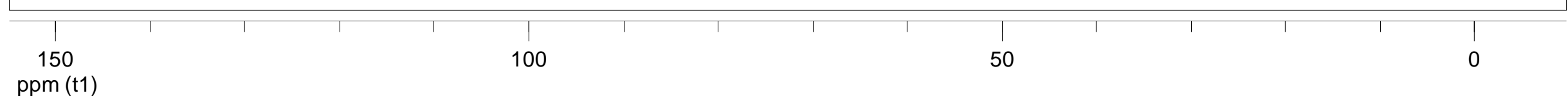




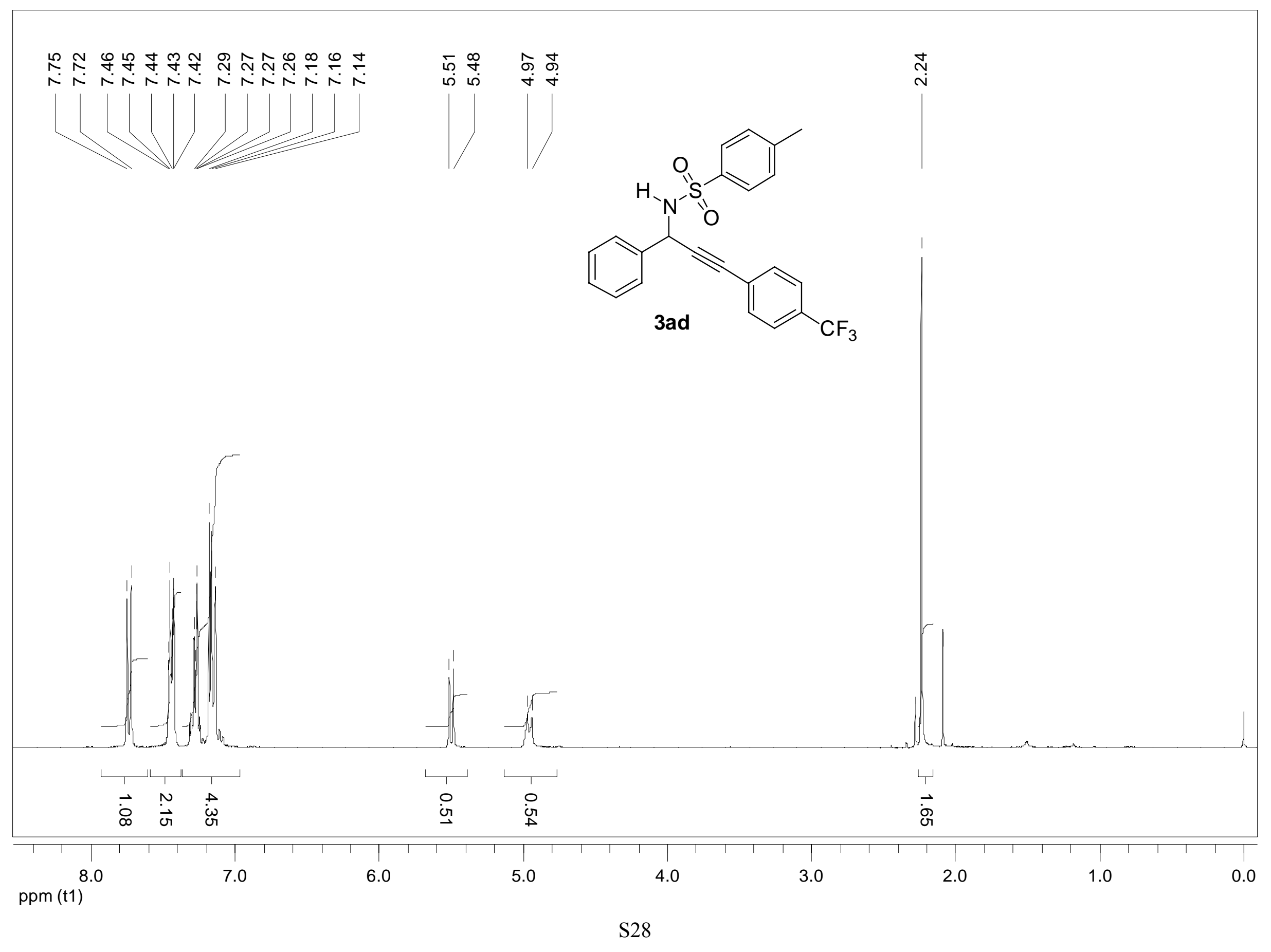




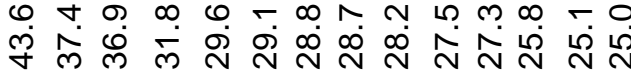
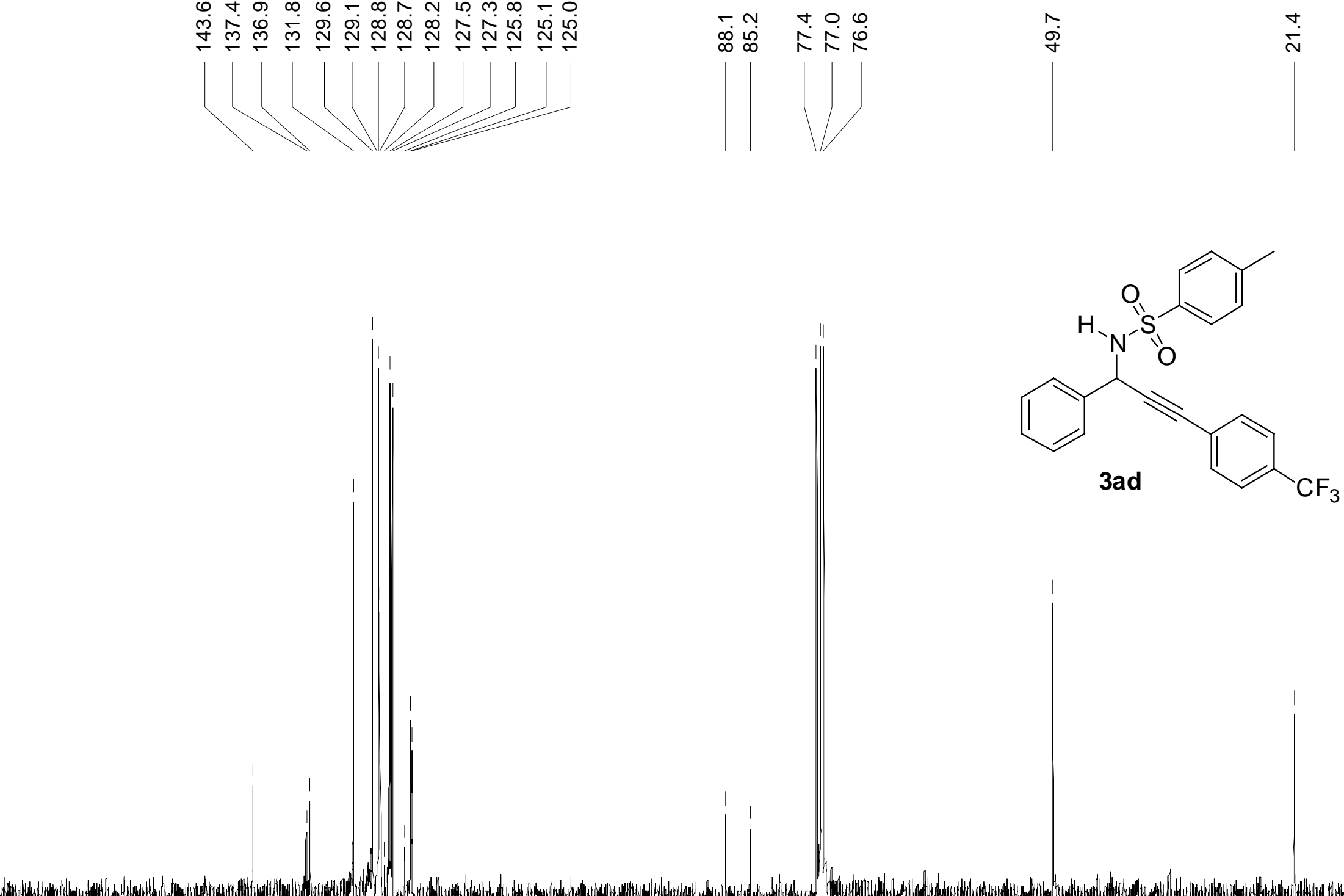

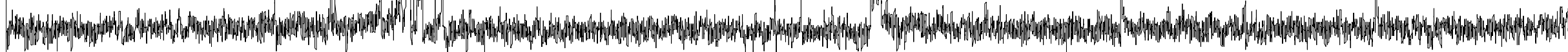

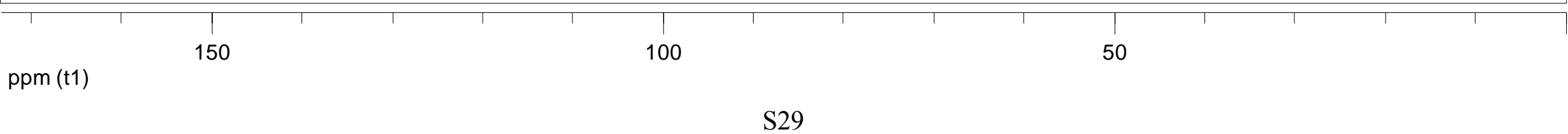




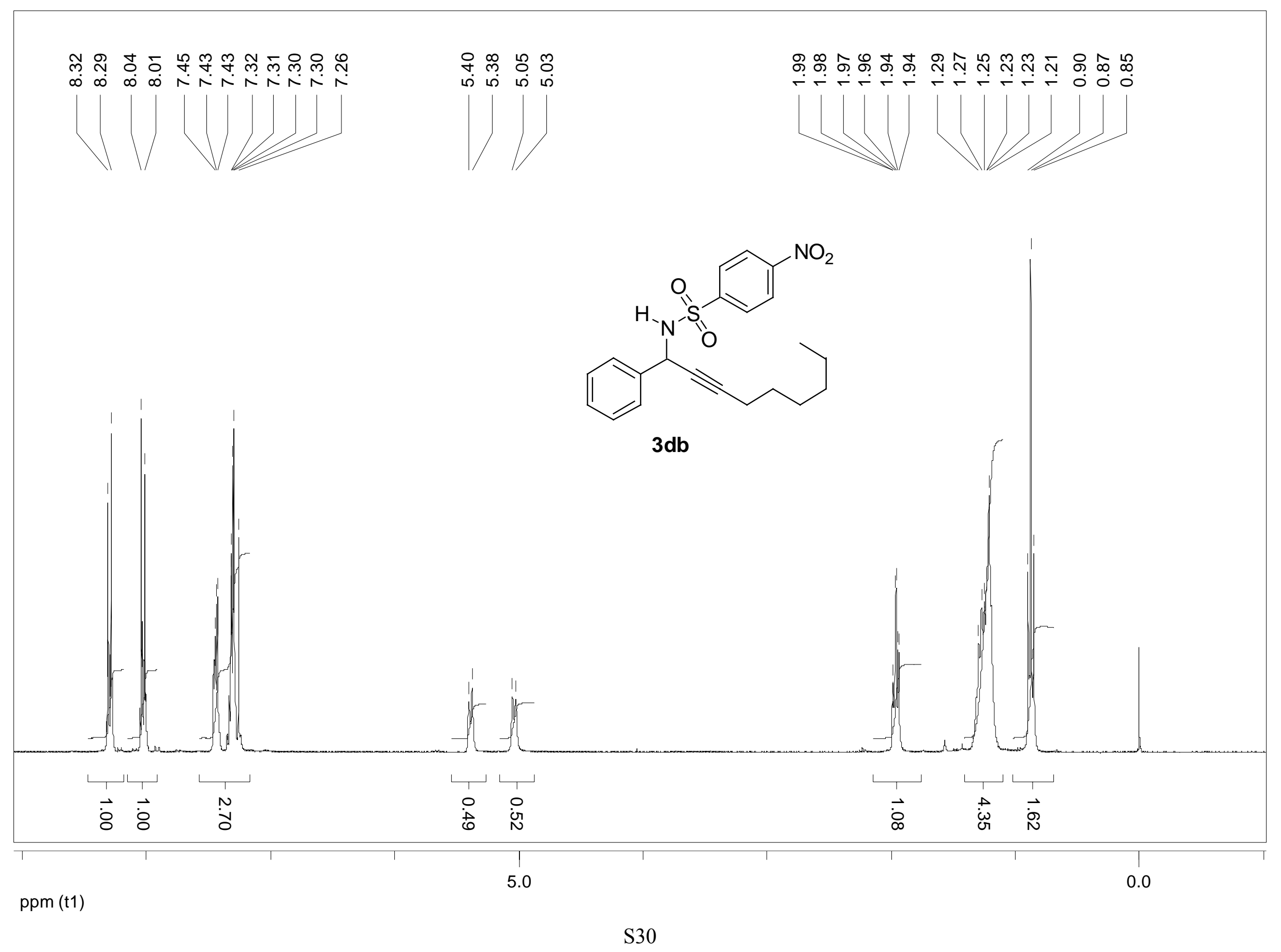



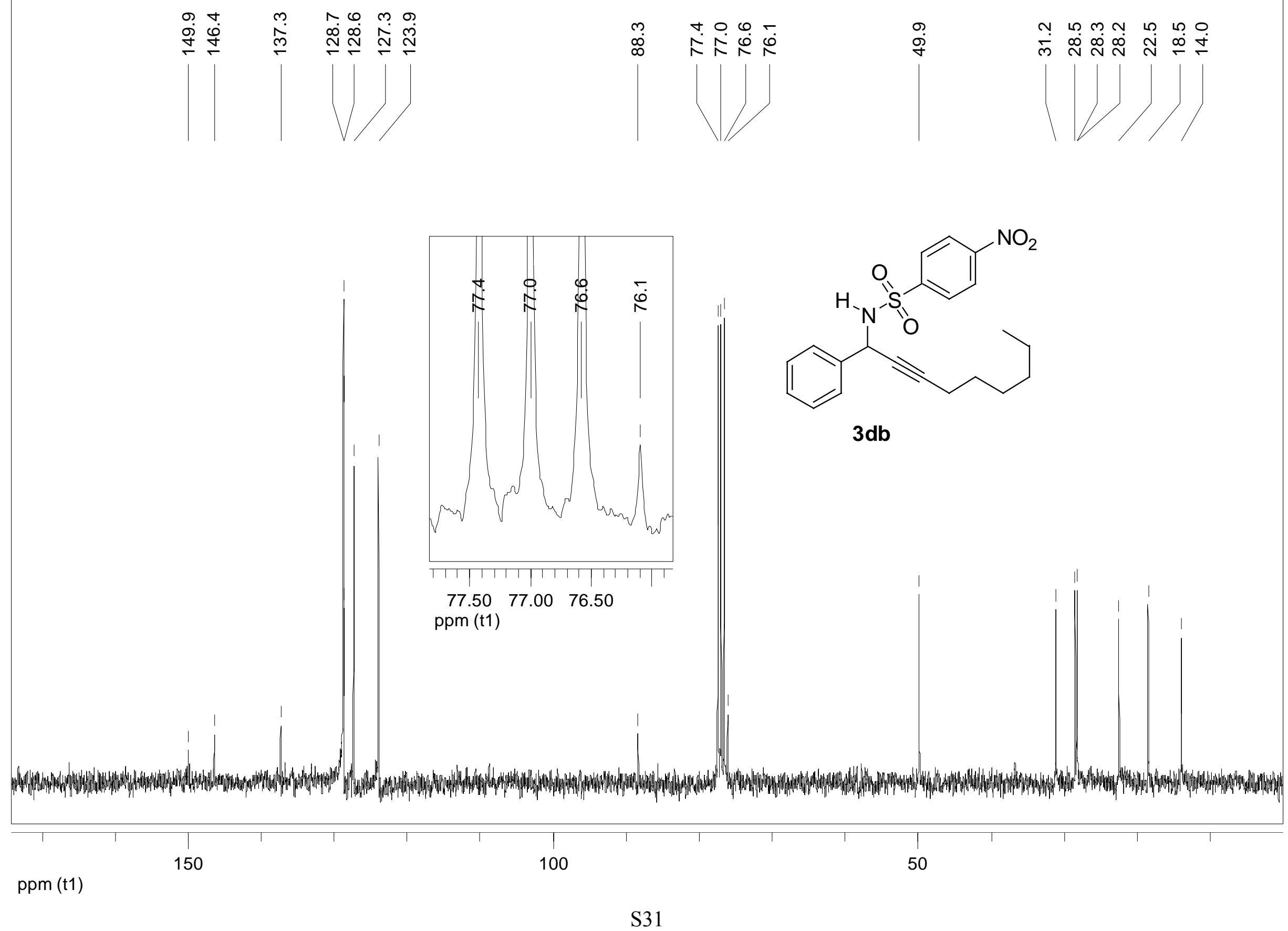


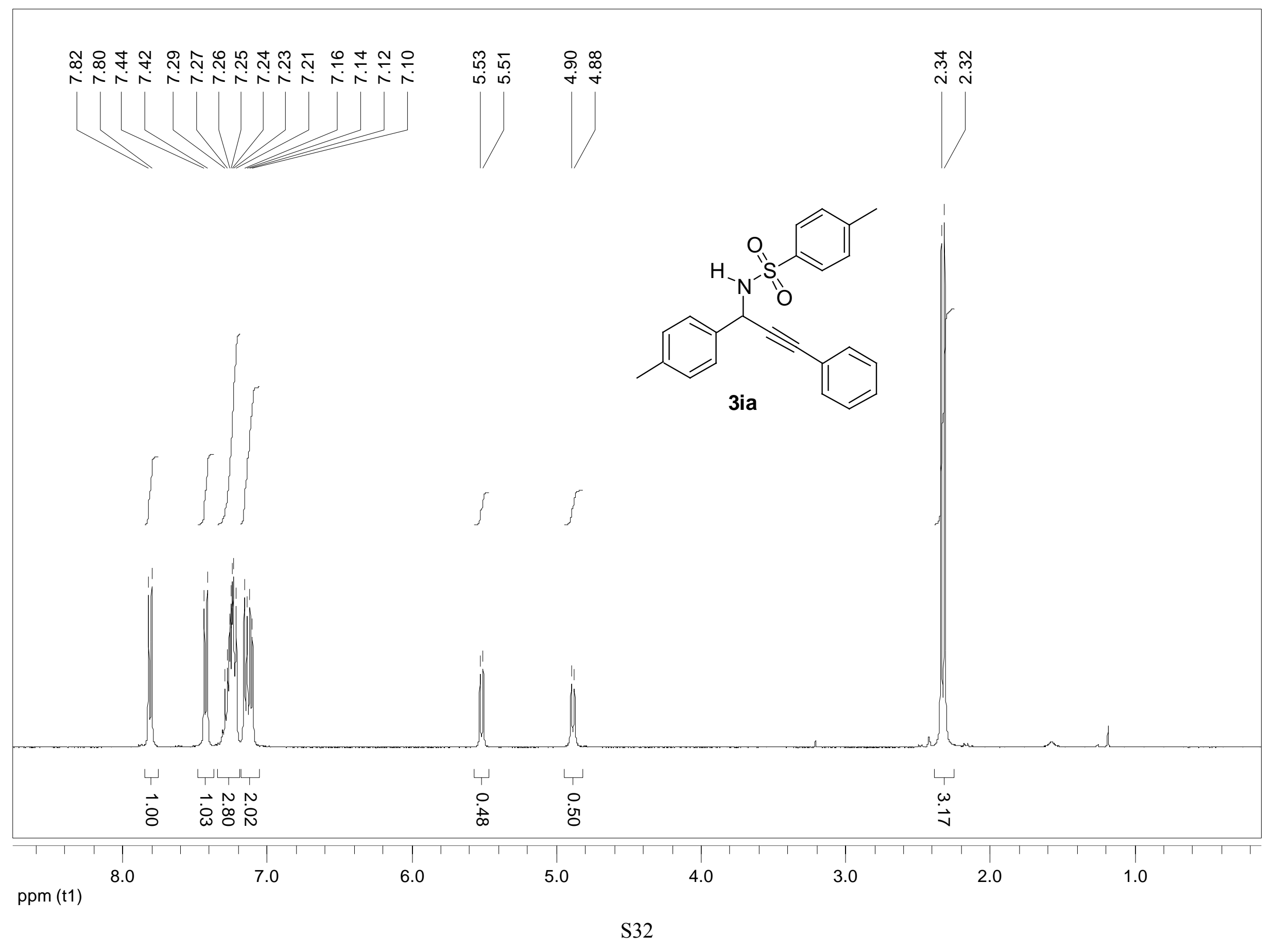



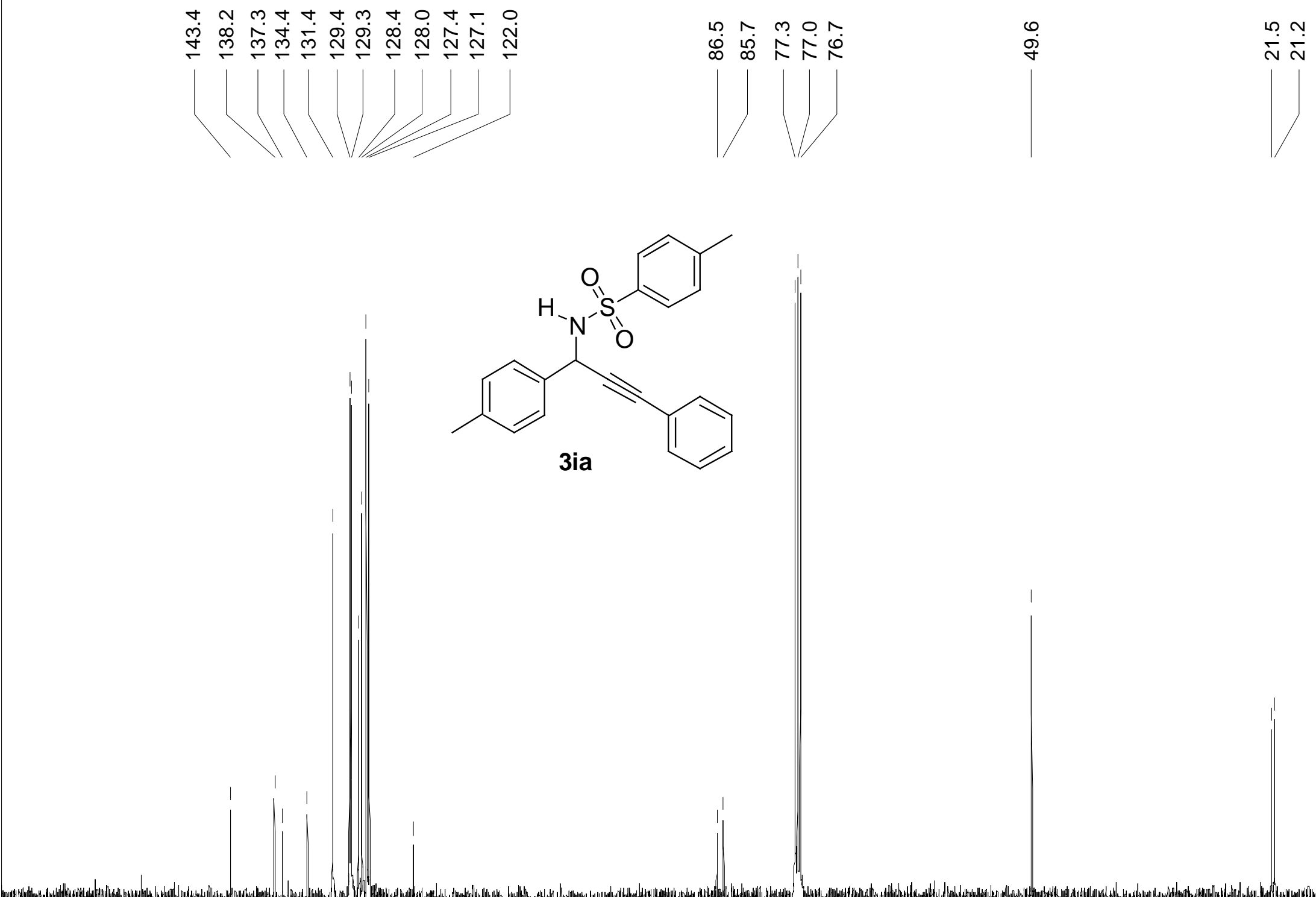

Jay ppm (t1) 


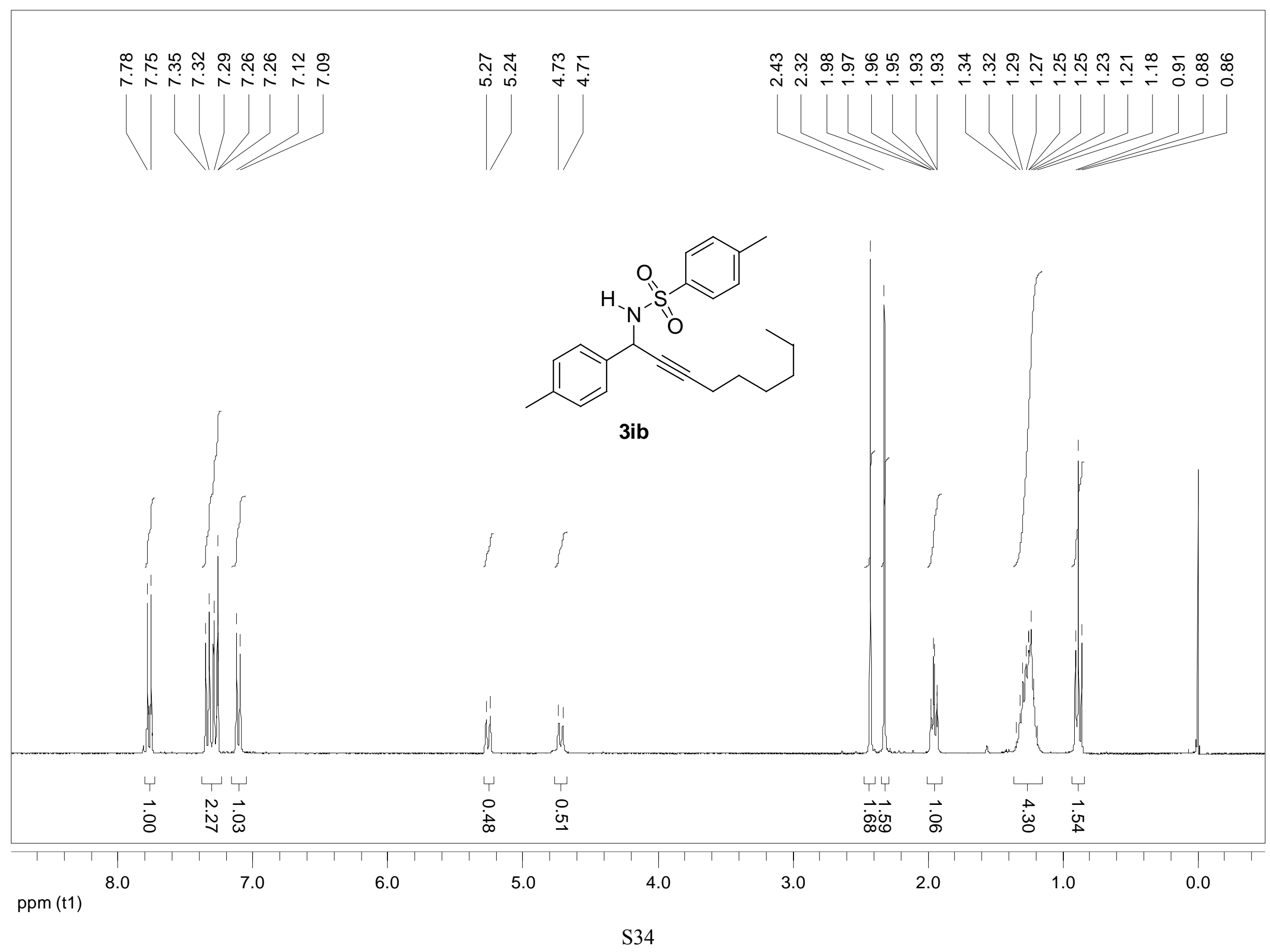




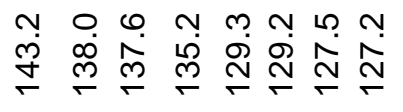
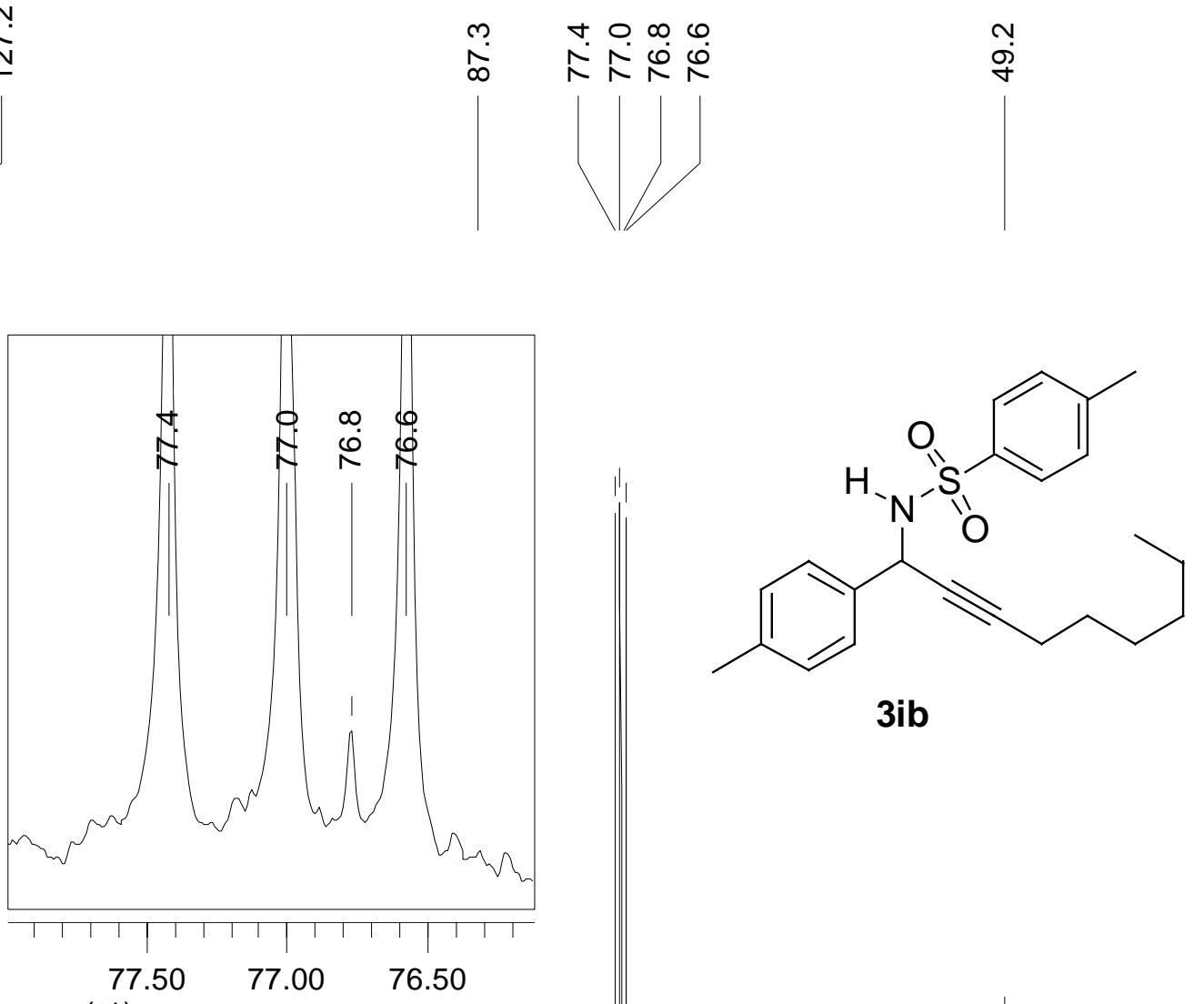

3ib

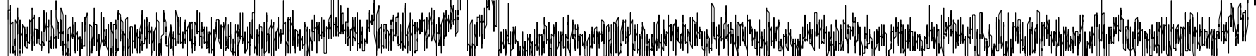

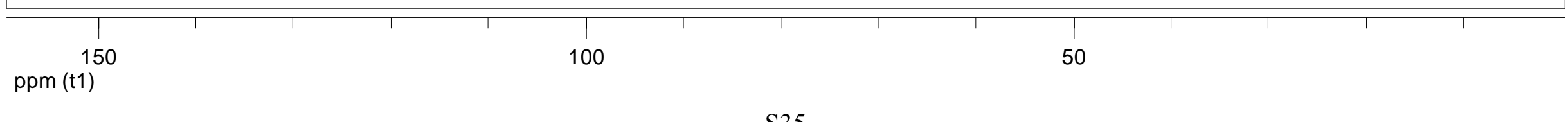




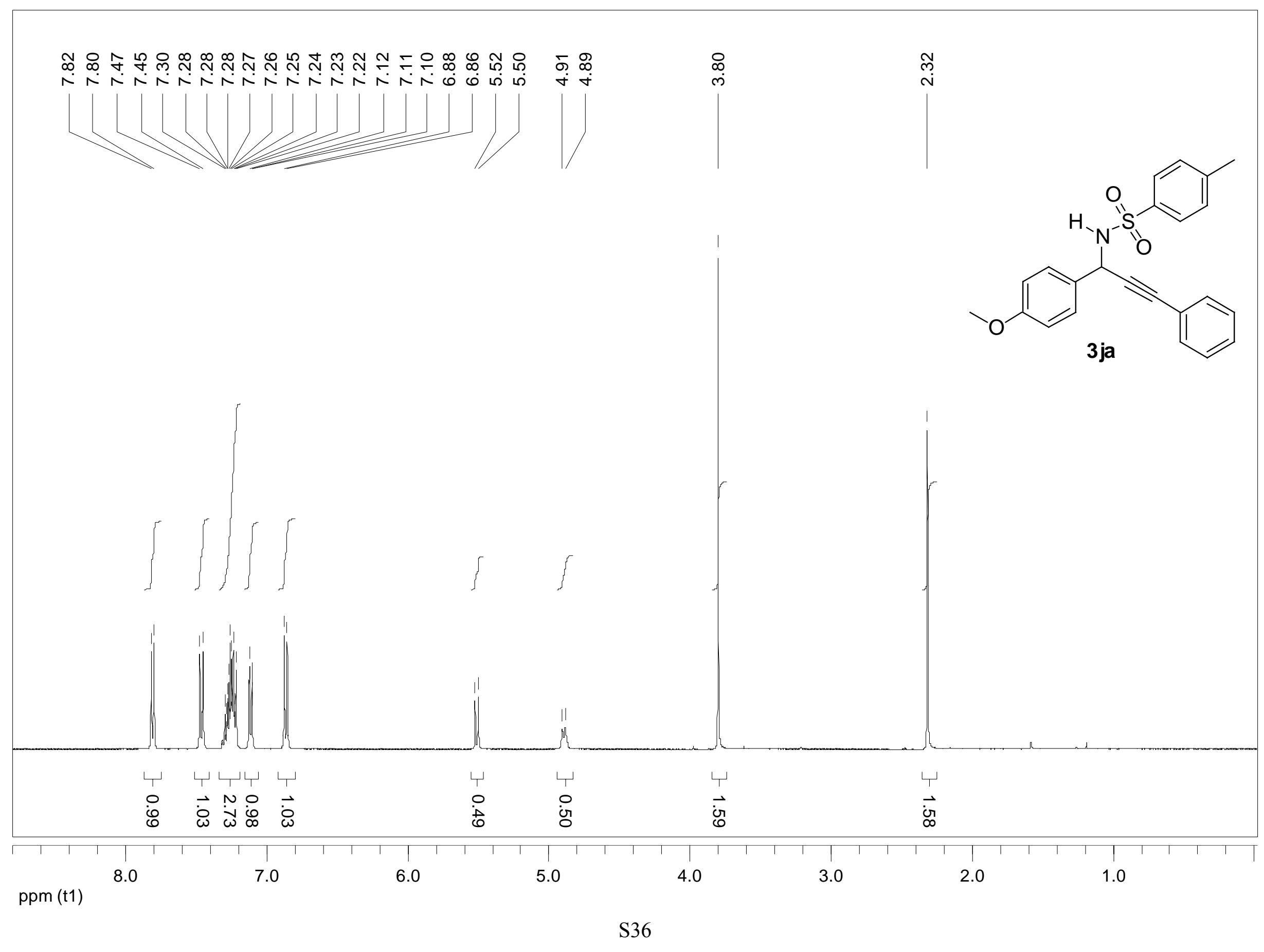



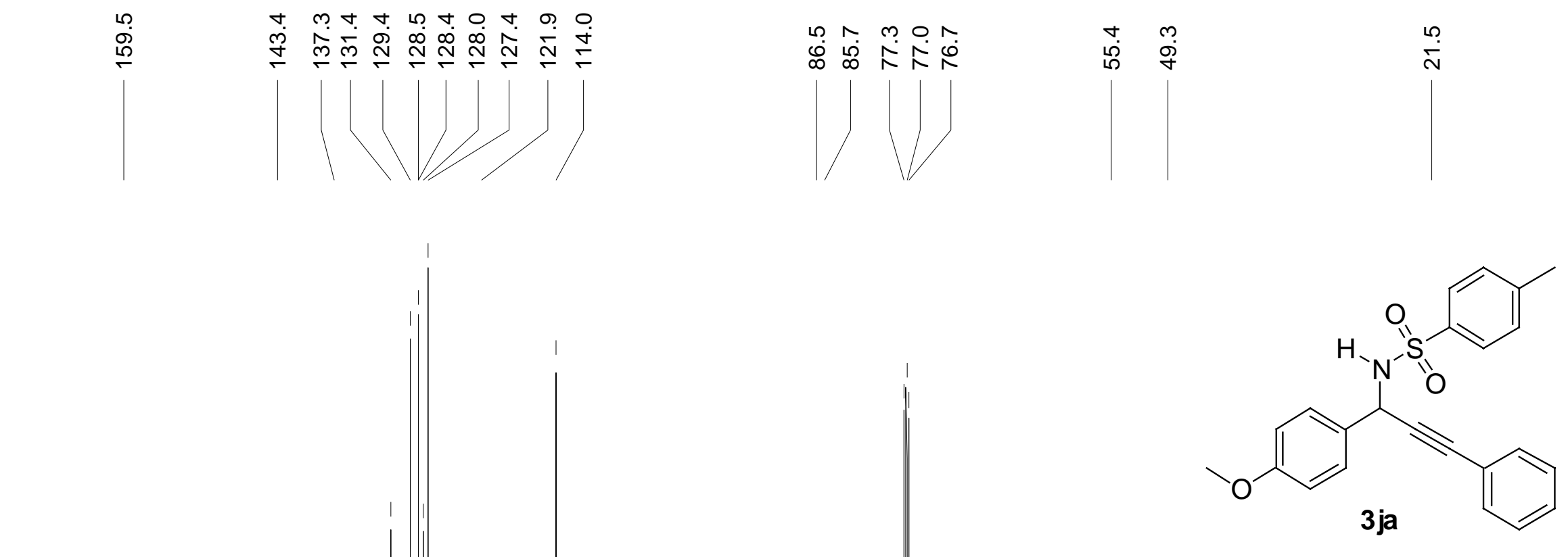

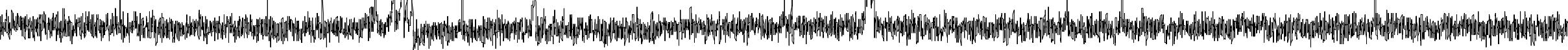

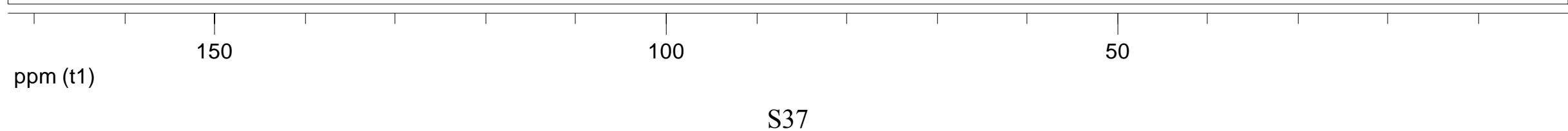




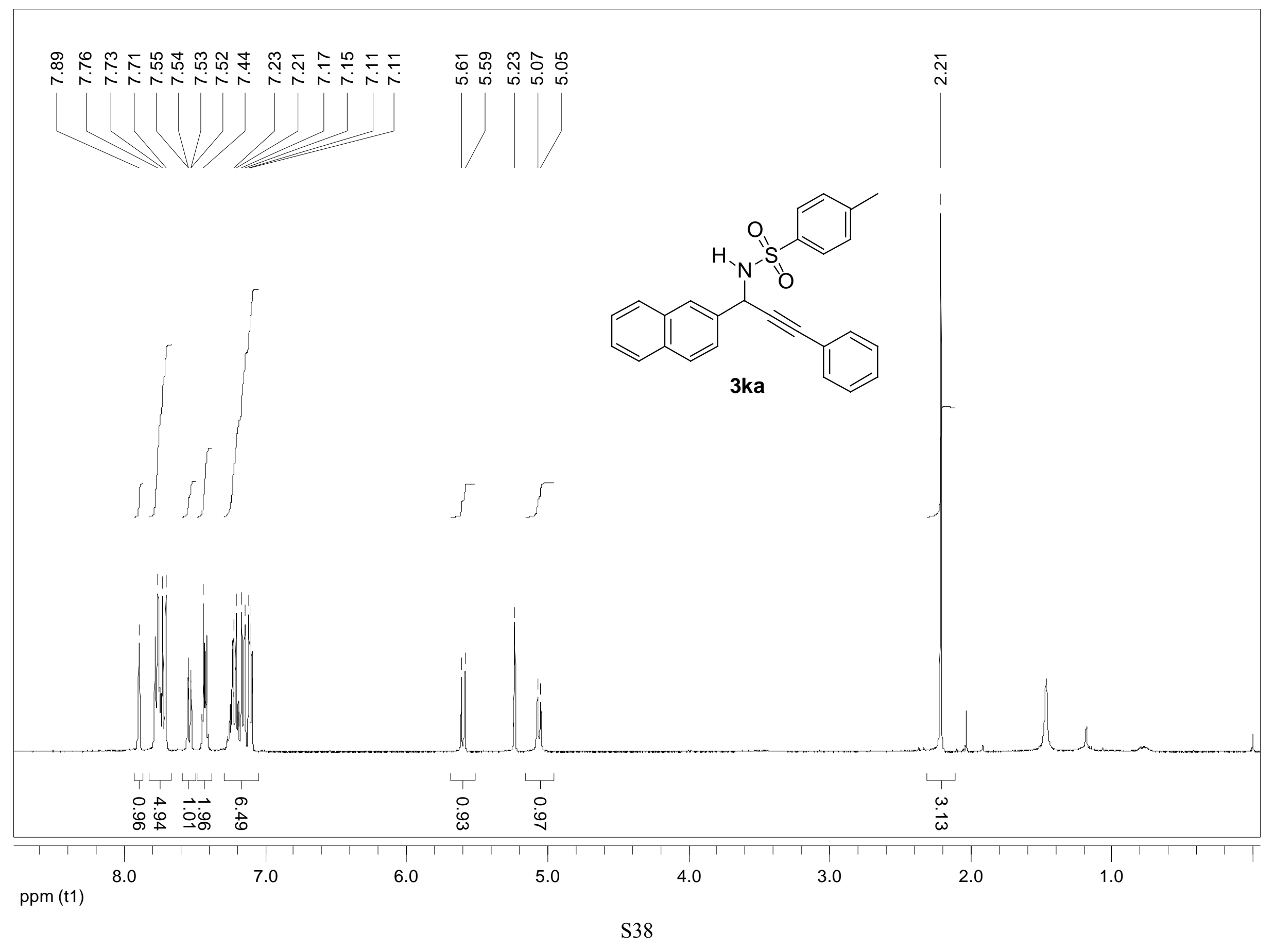




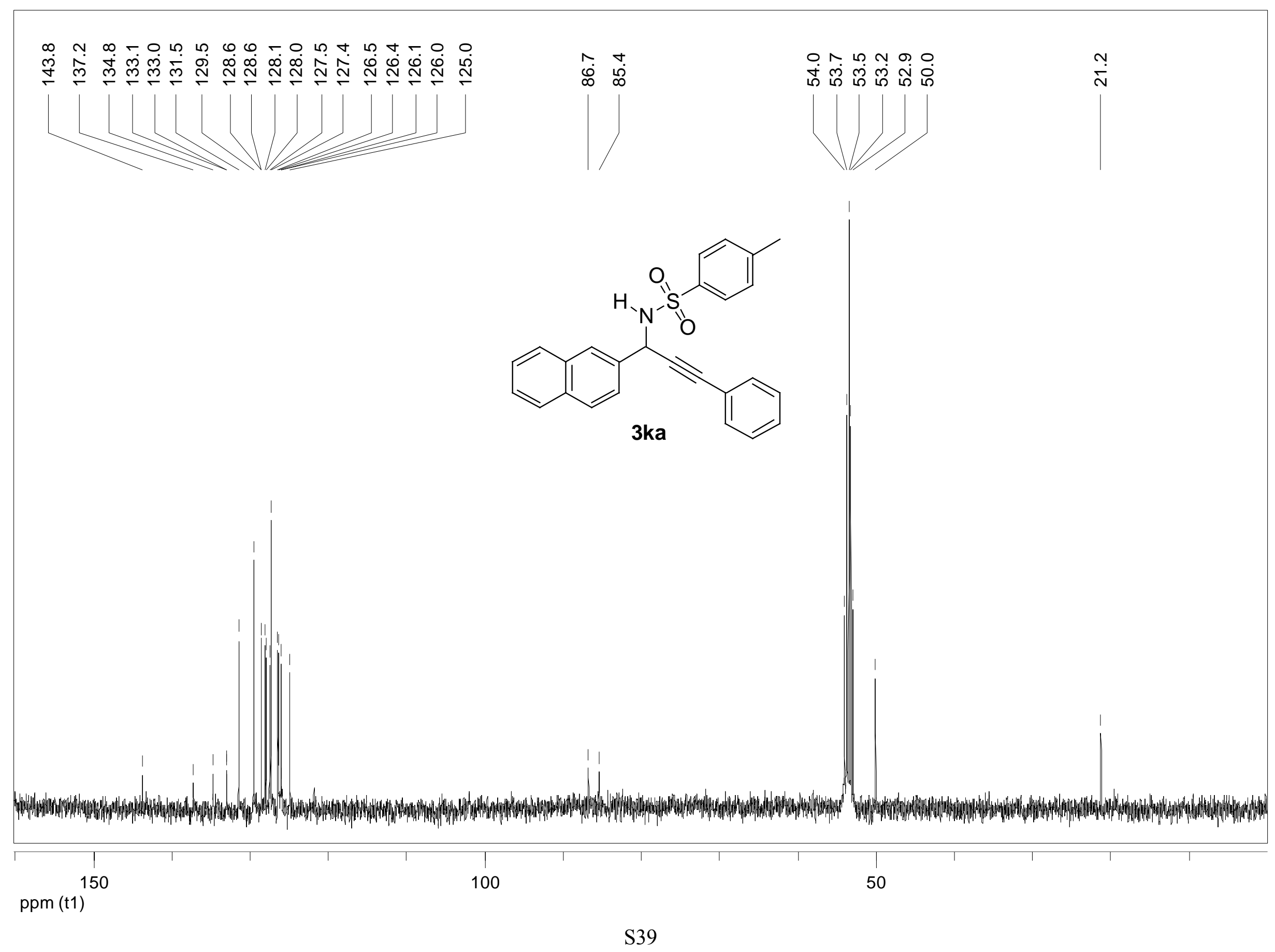




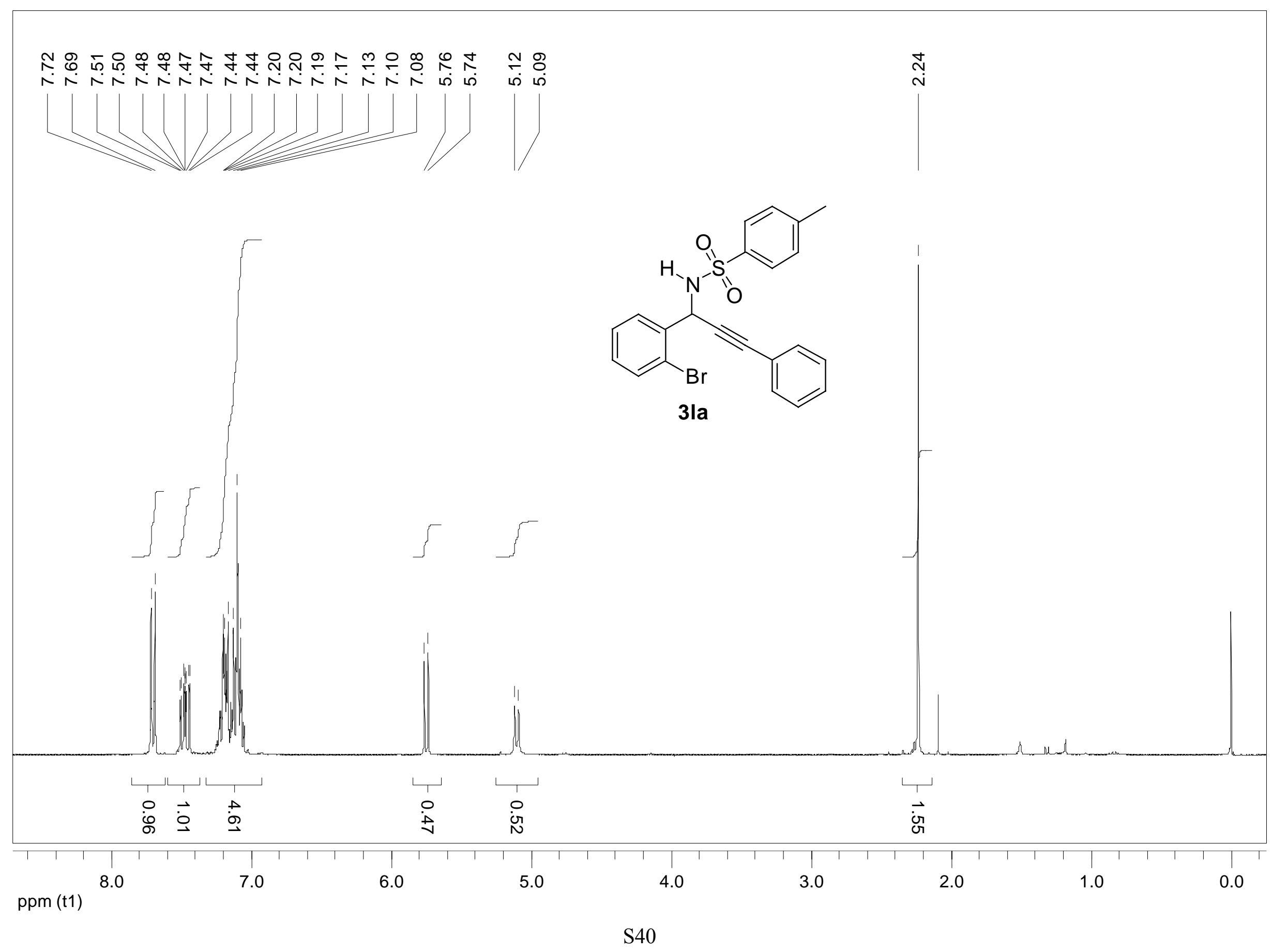


เ ก ก เ เ

离 $\stackrel{m}{\rightarrow}$

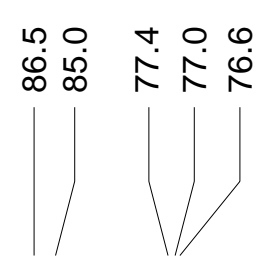

웅

$\stackrel{+}{\mathrm{A}}$

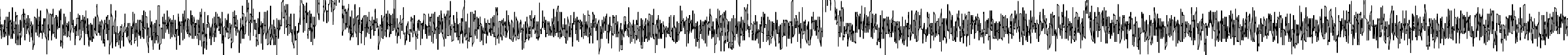
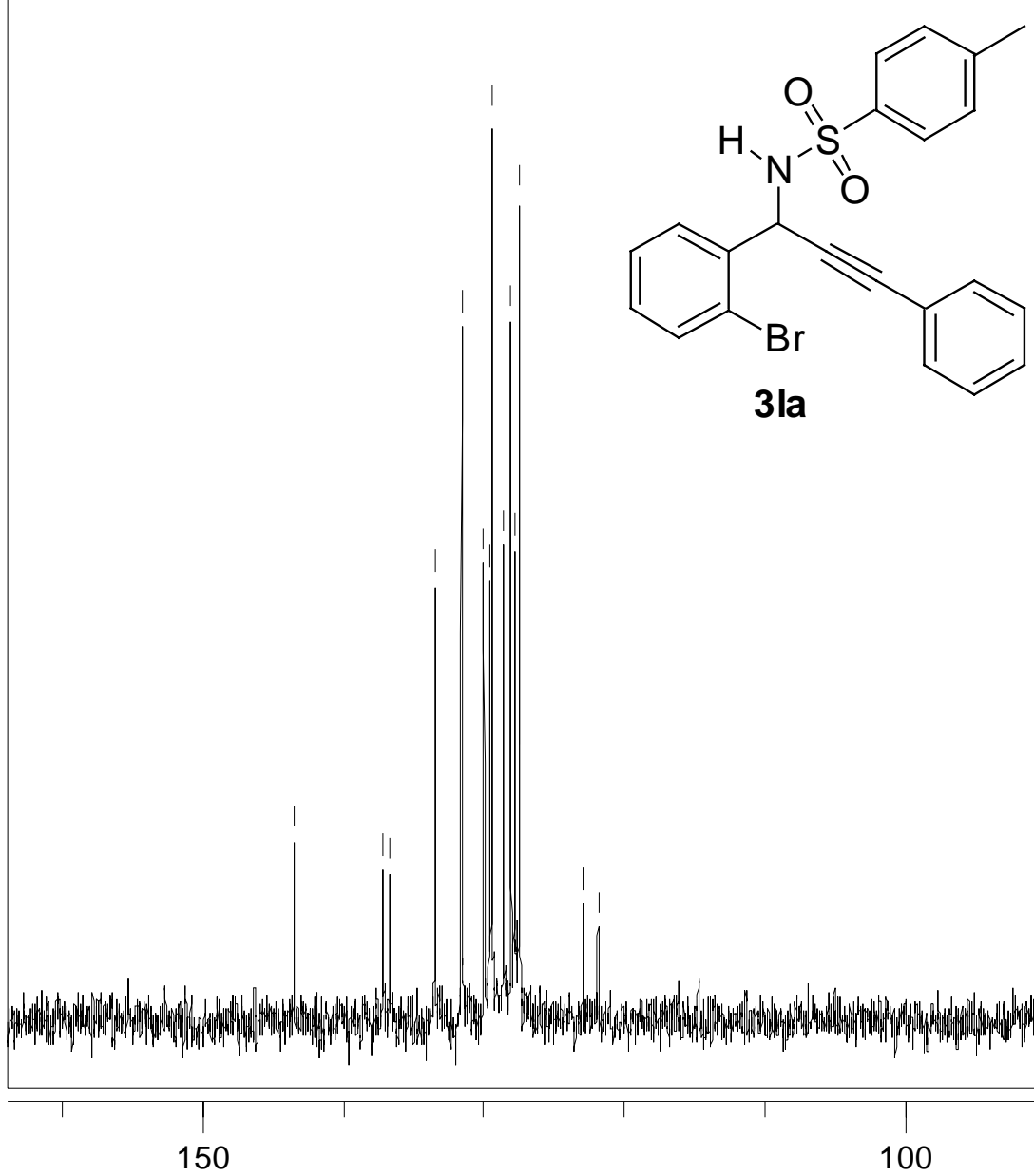

150

100

ppm (t1) 


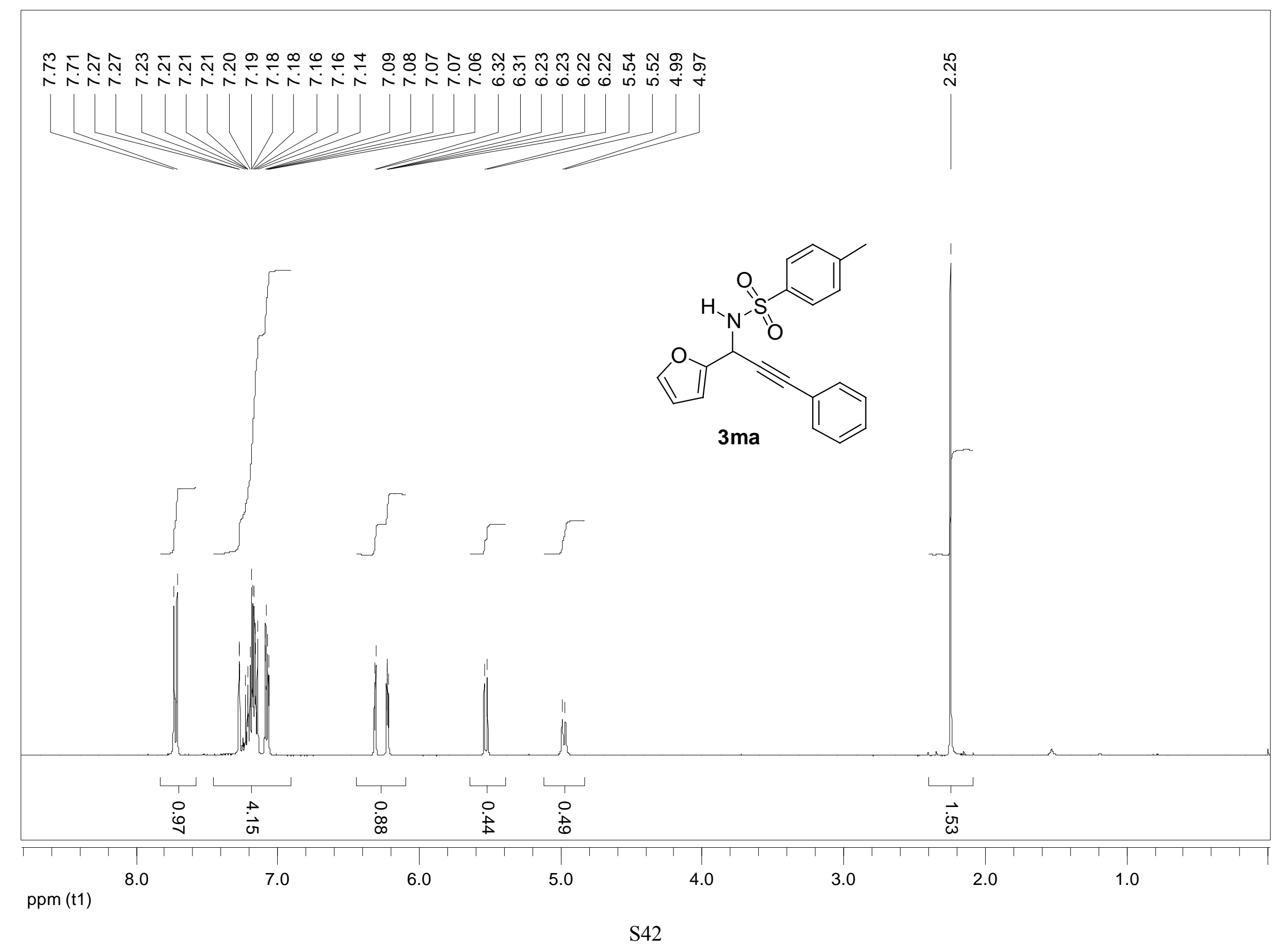




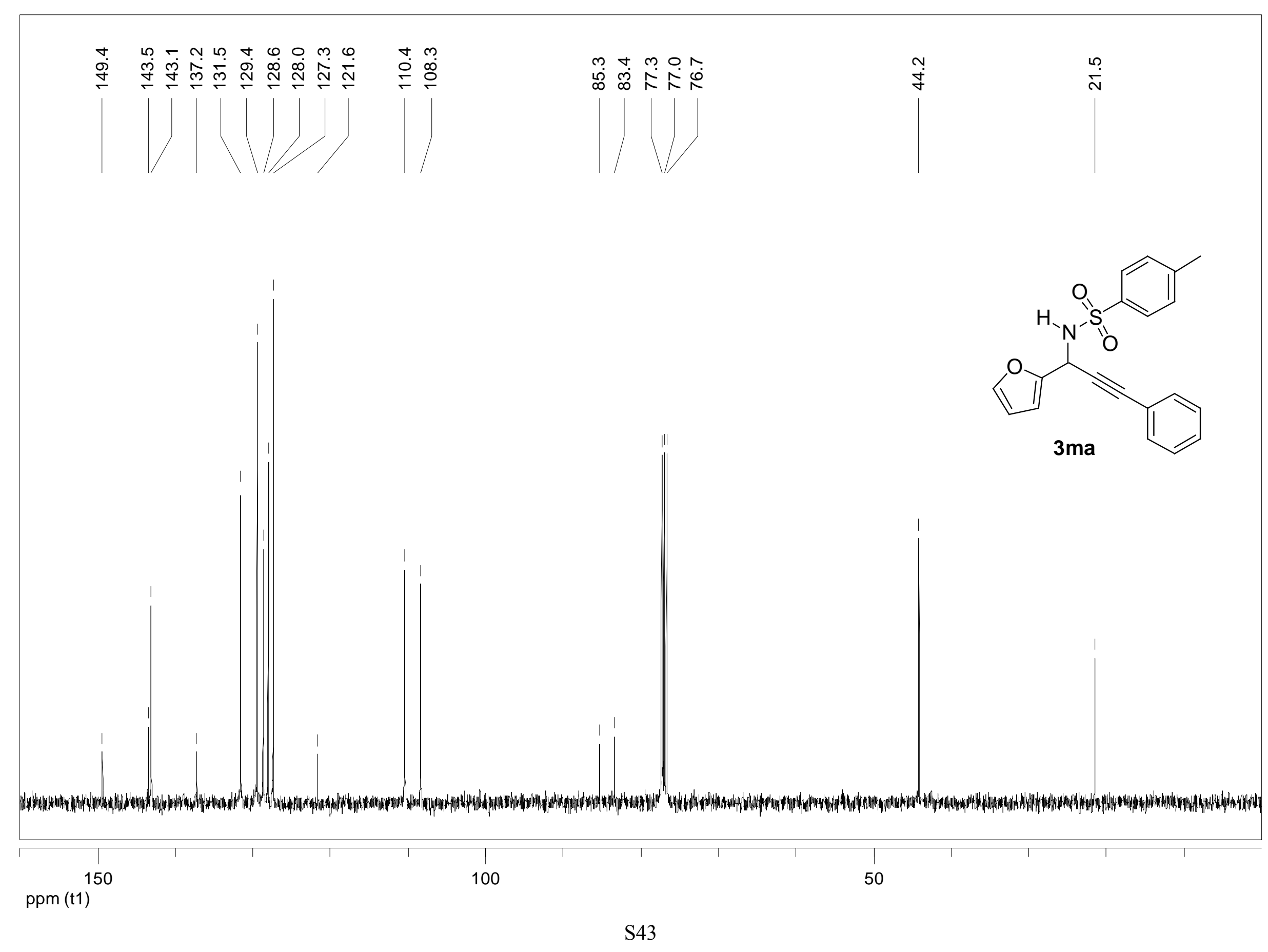




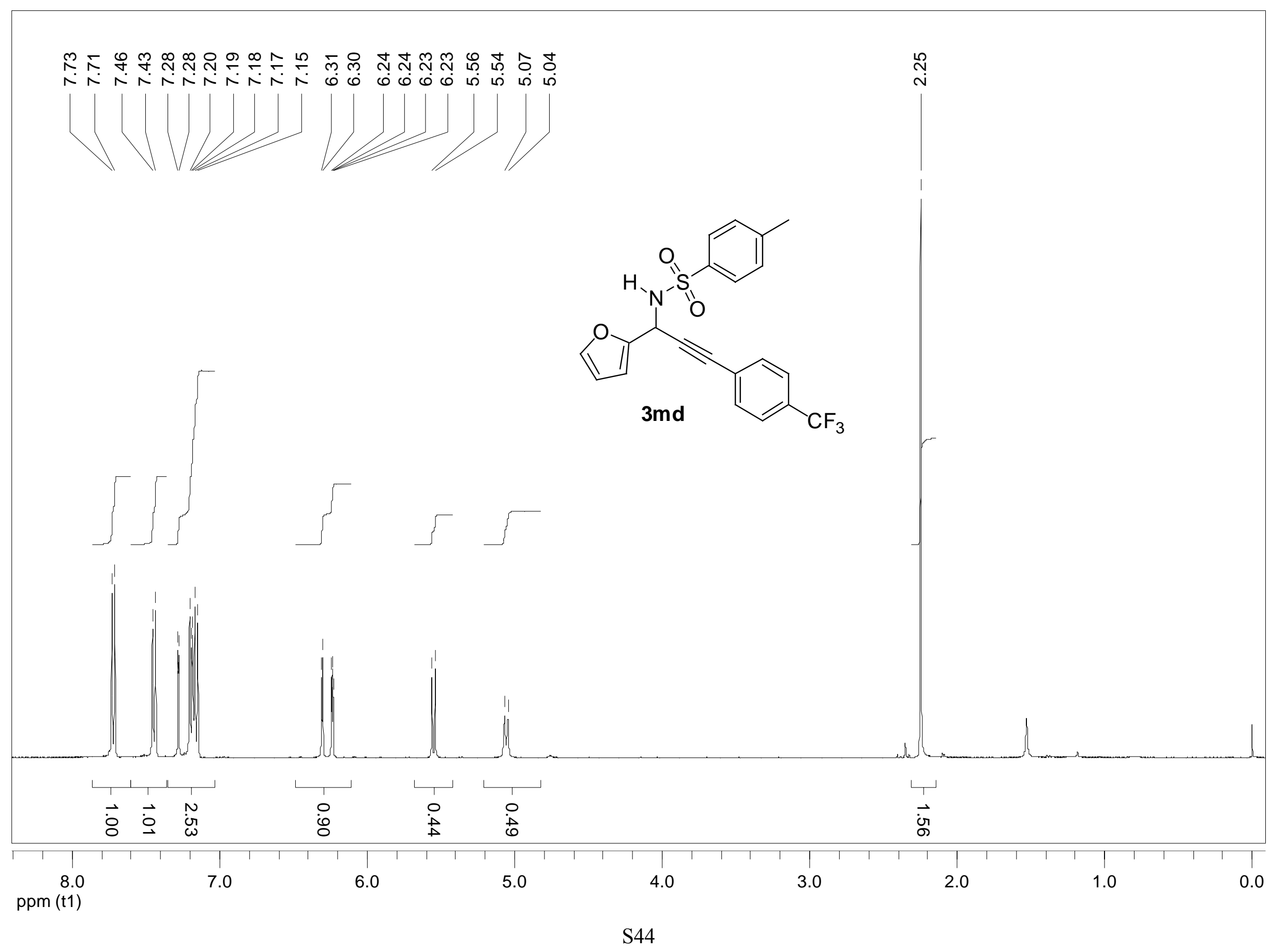




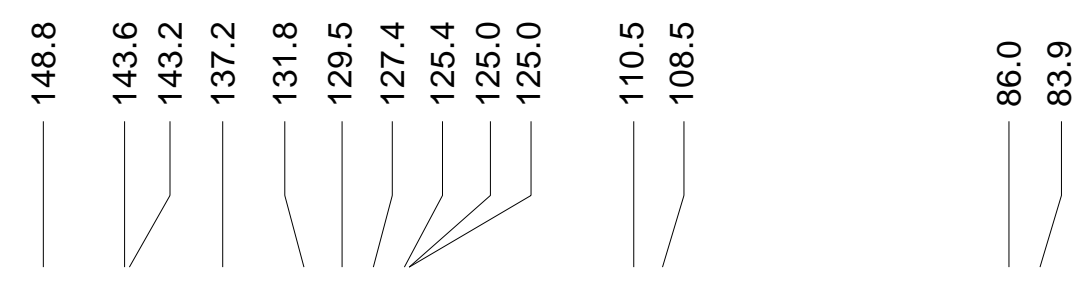

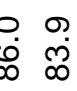
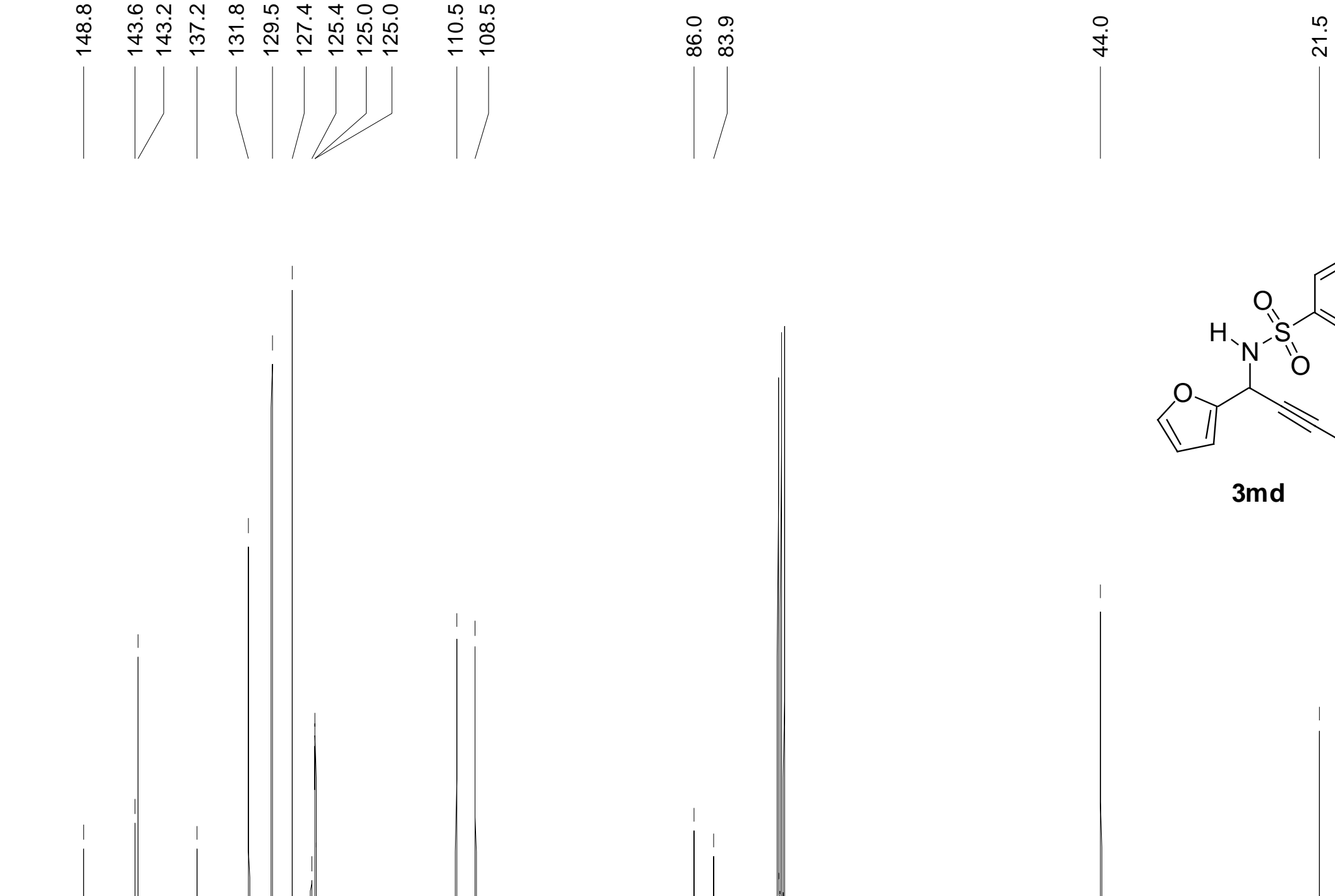

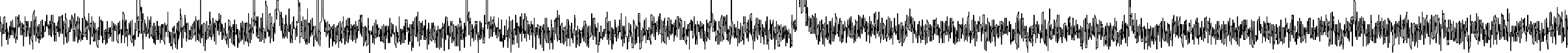
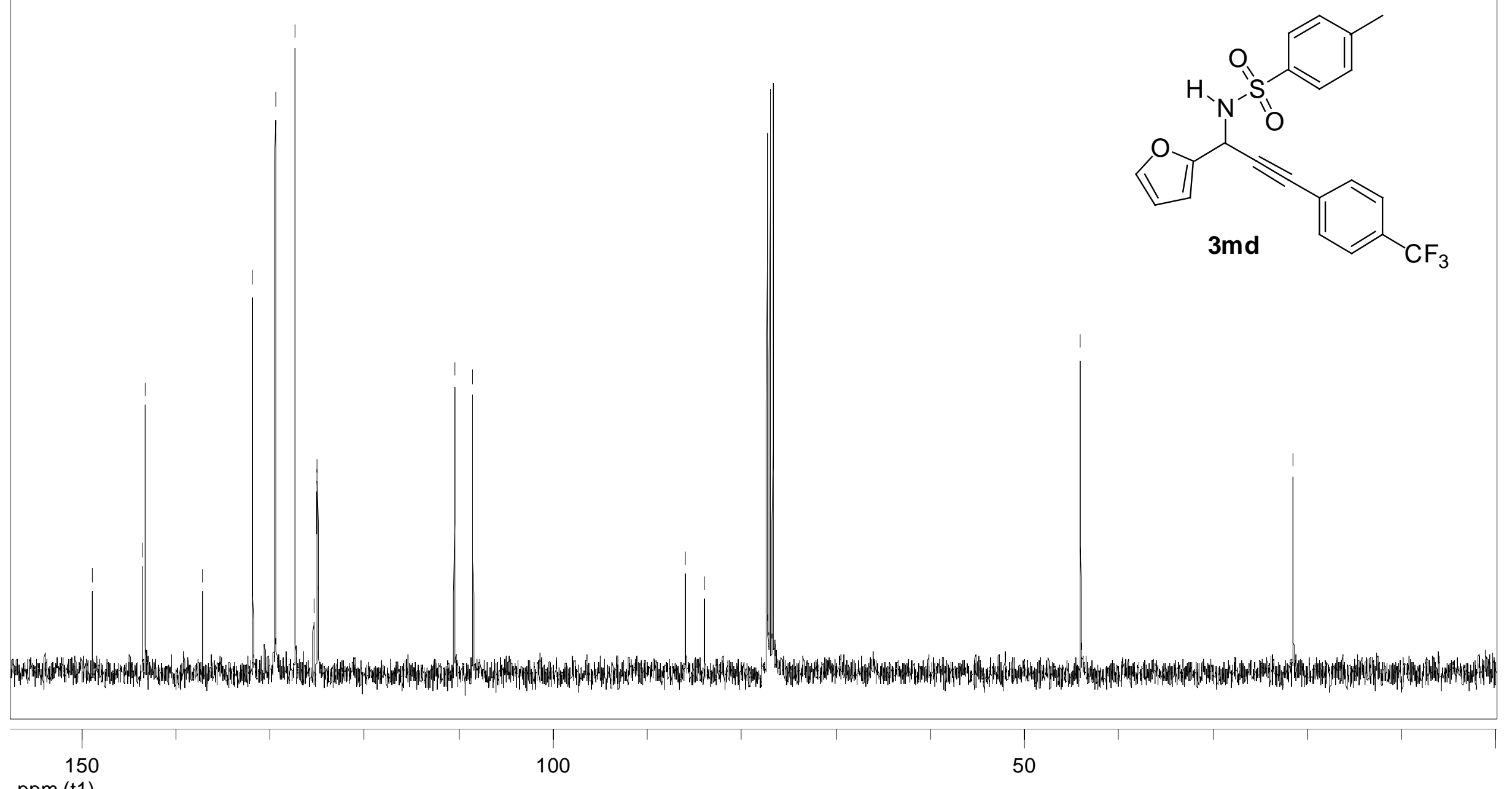

ppm (t1) 


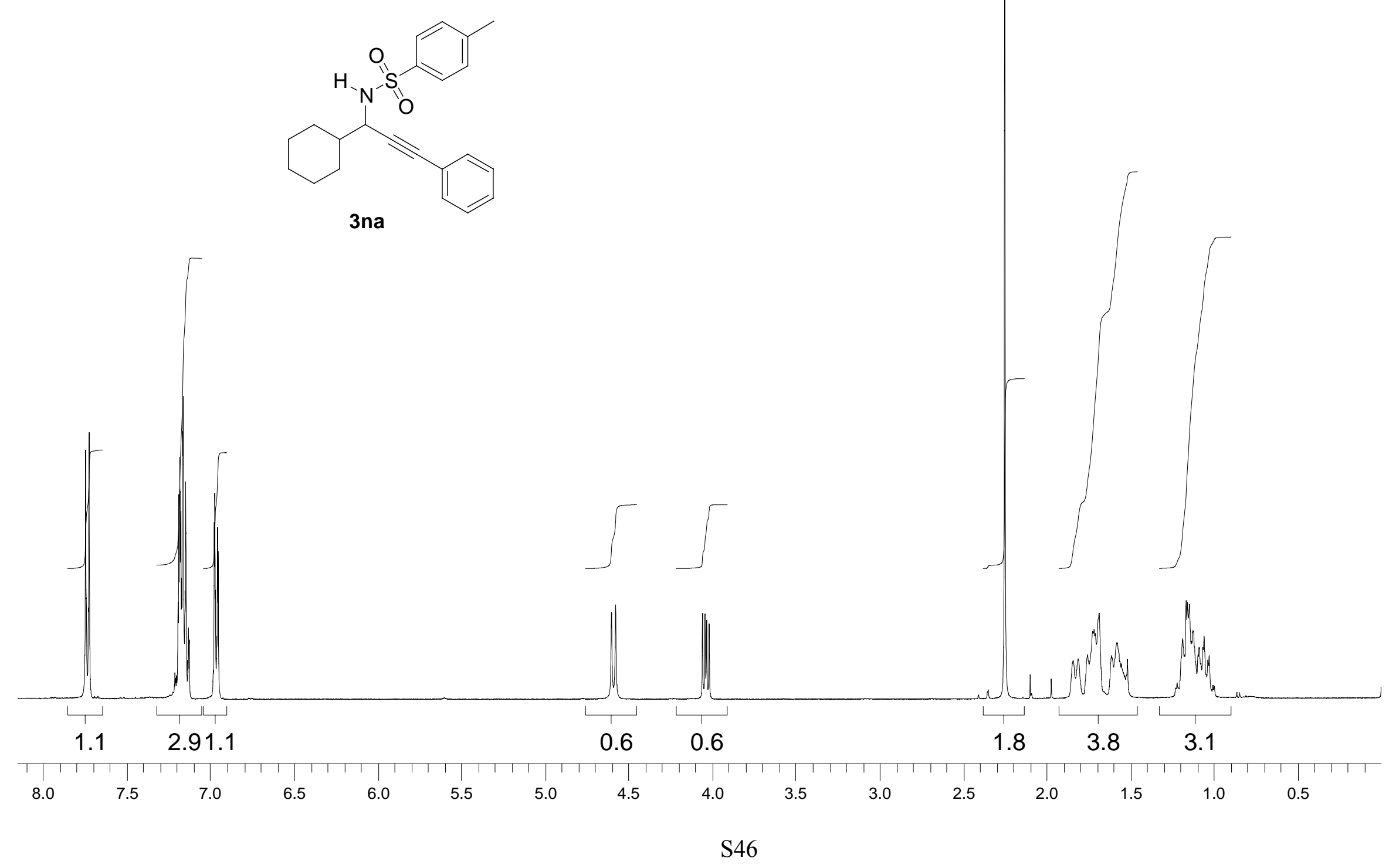




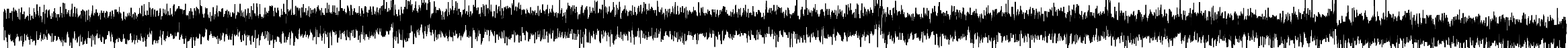

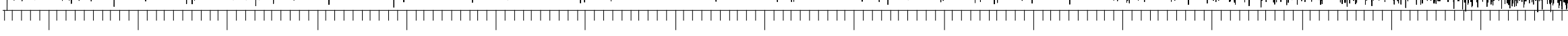



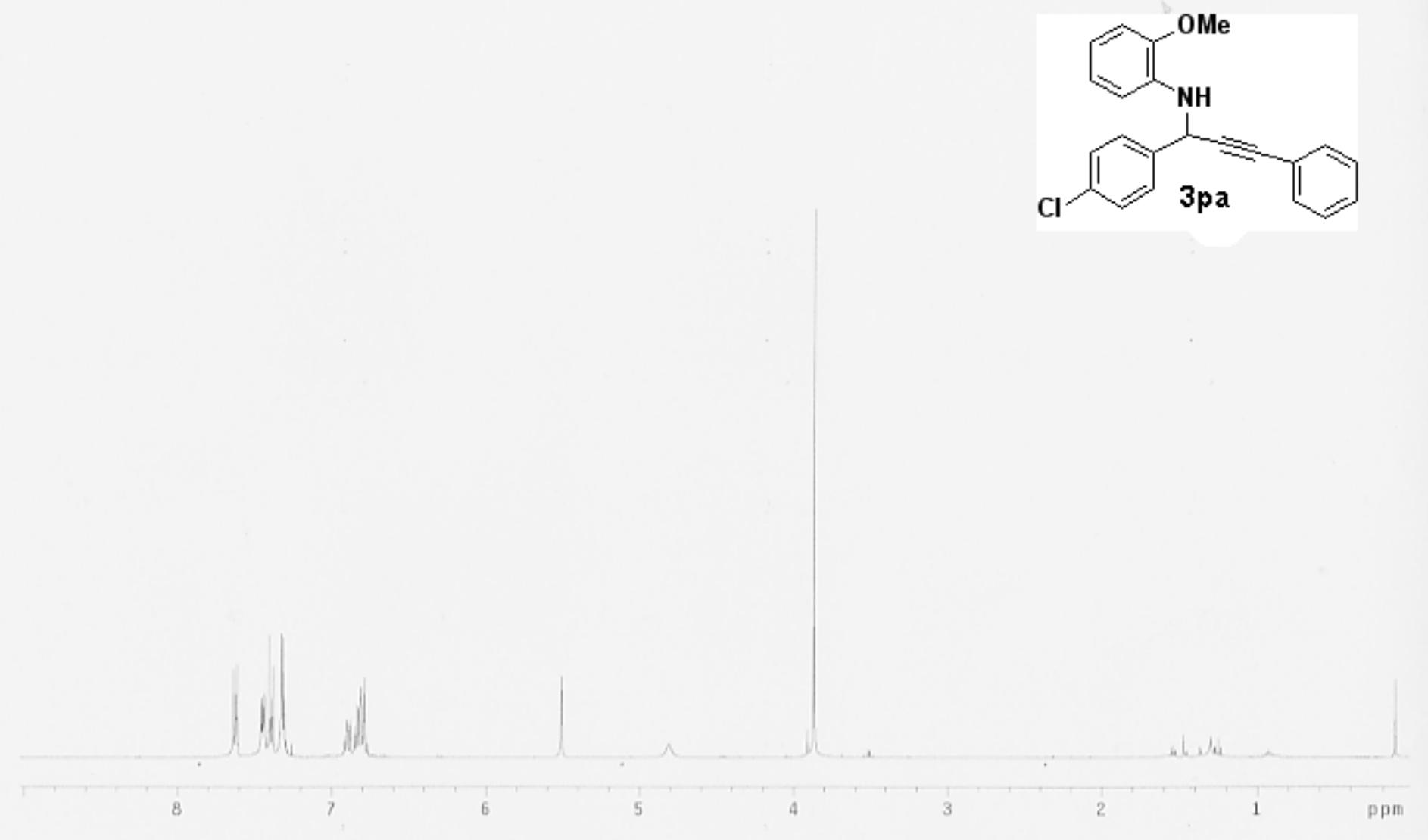

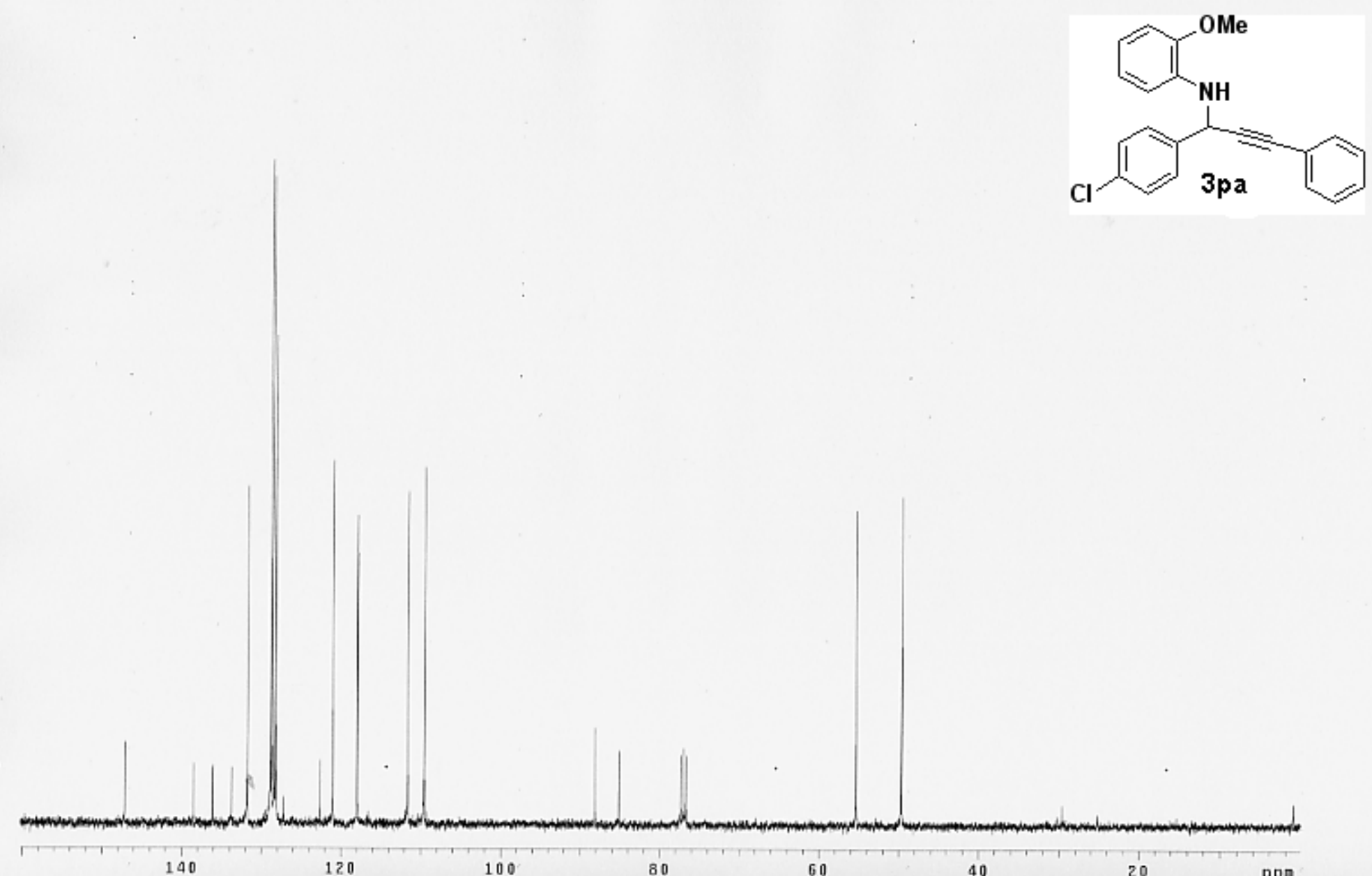
S50

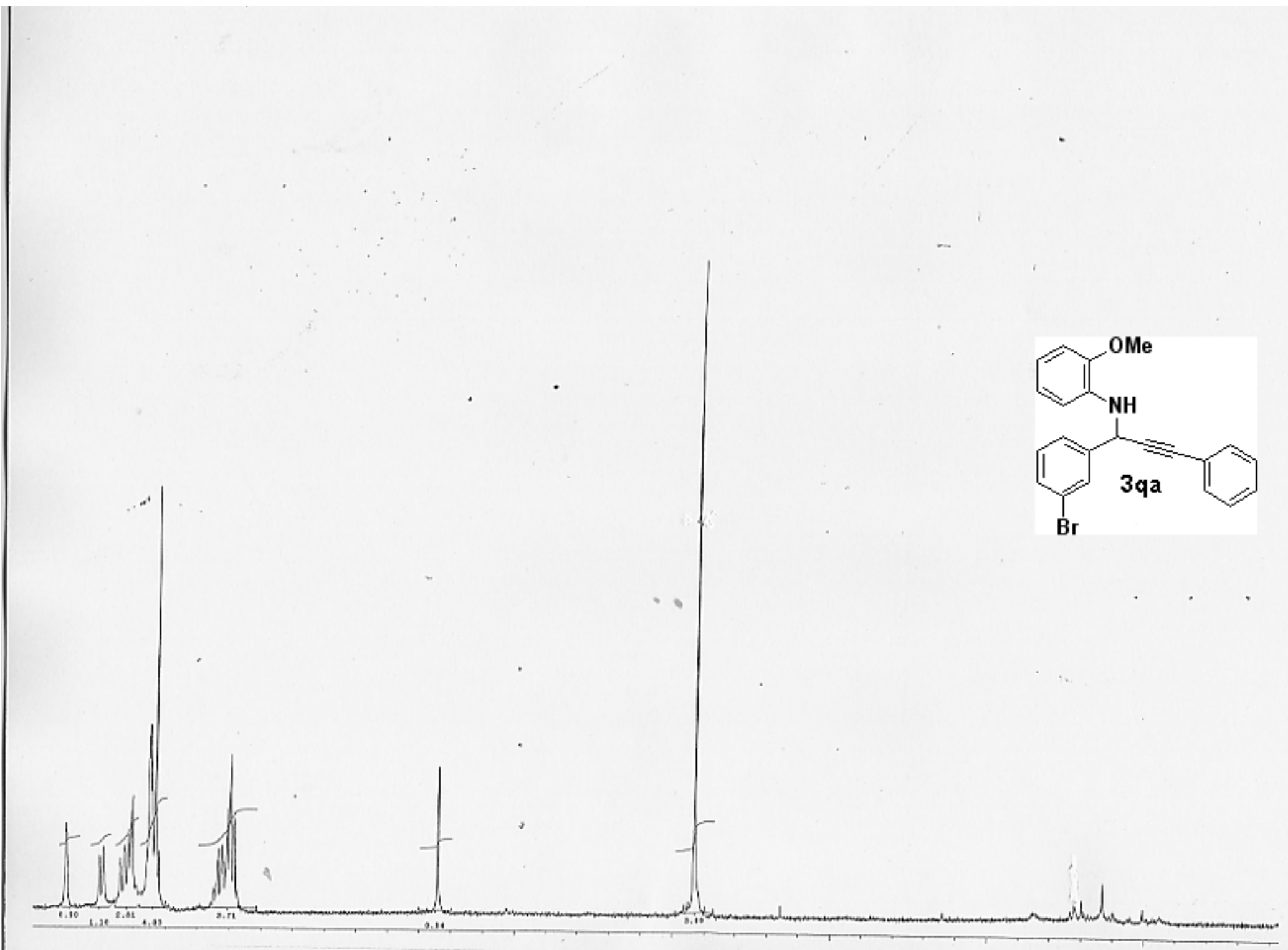

S50 


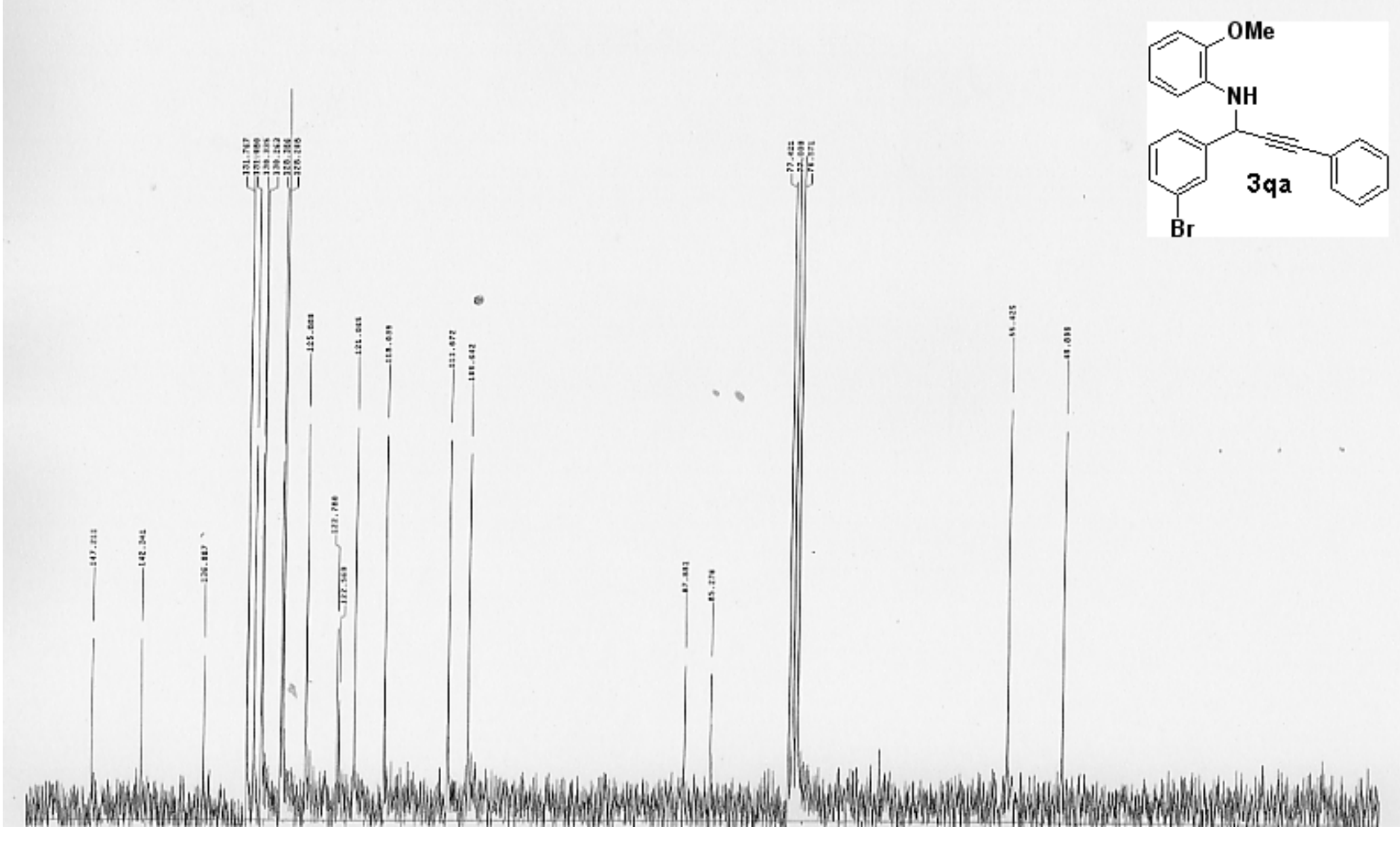


अ.

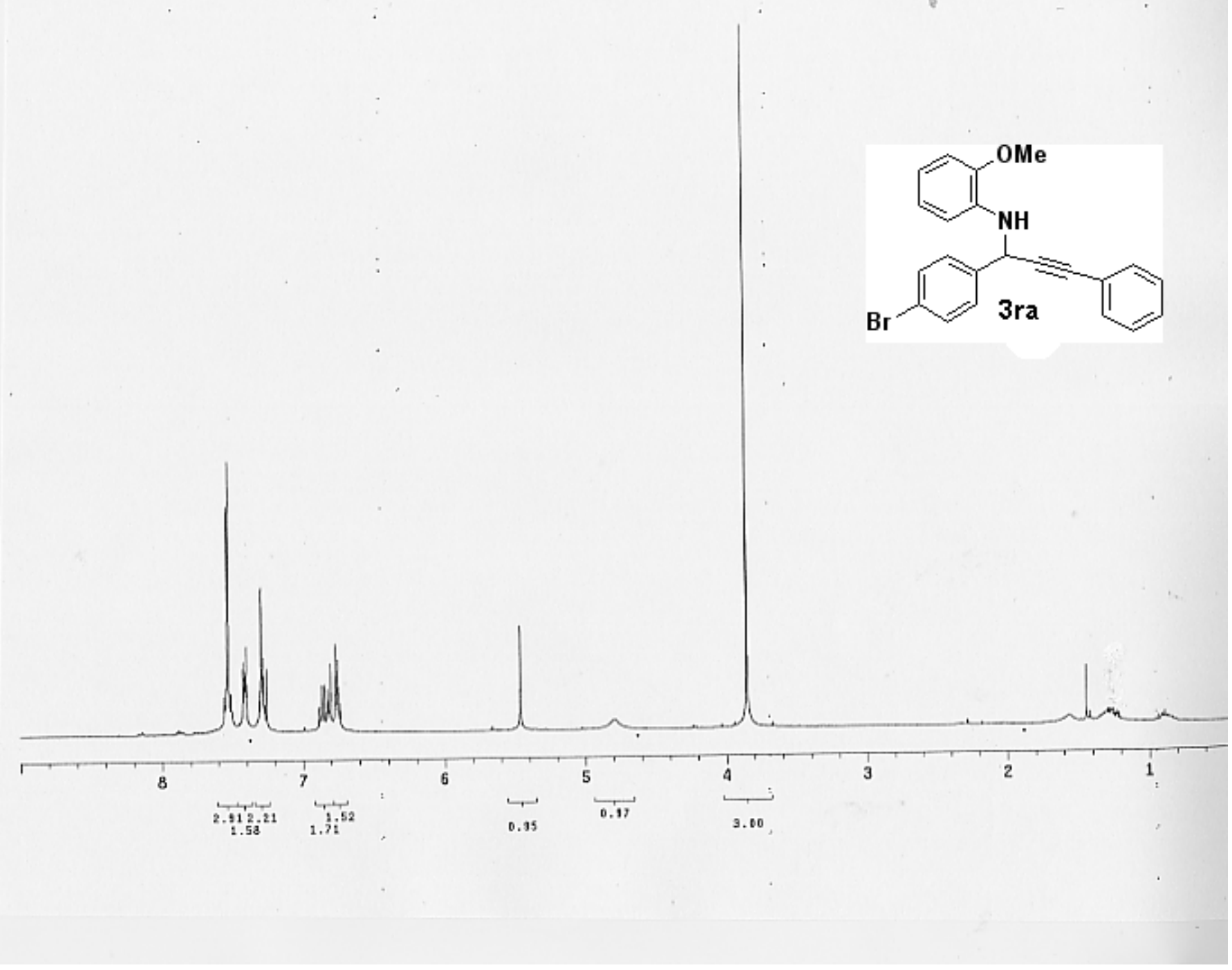



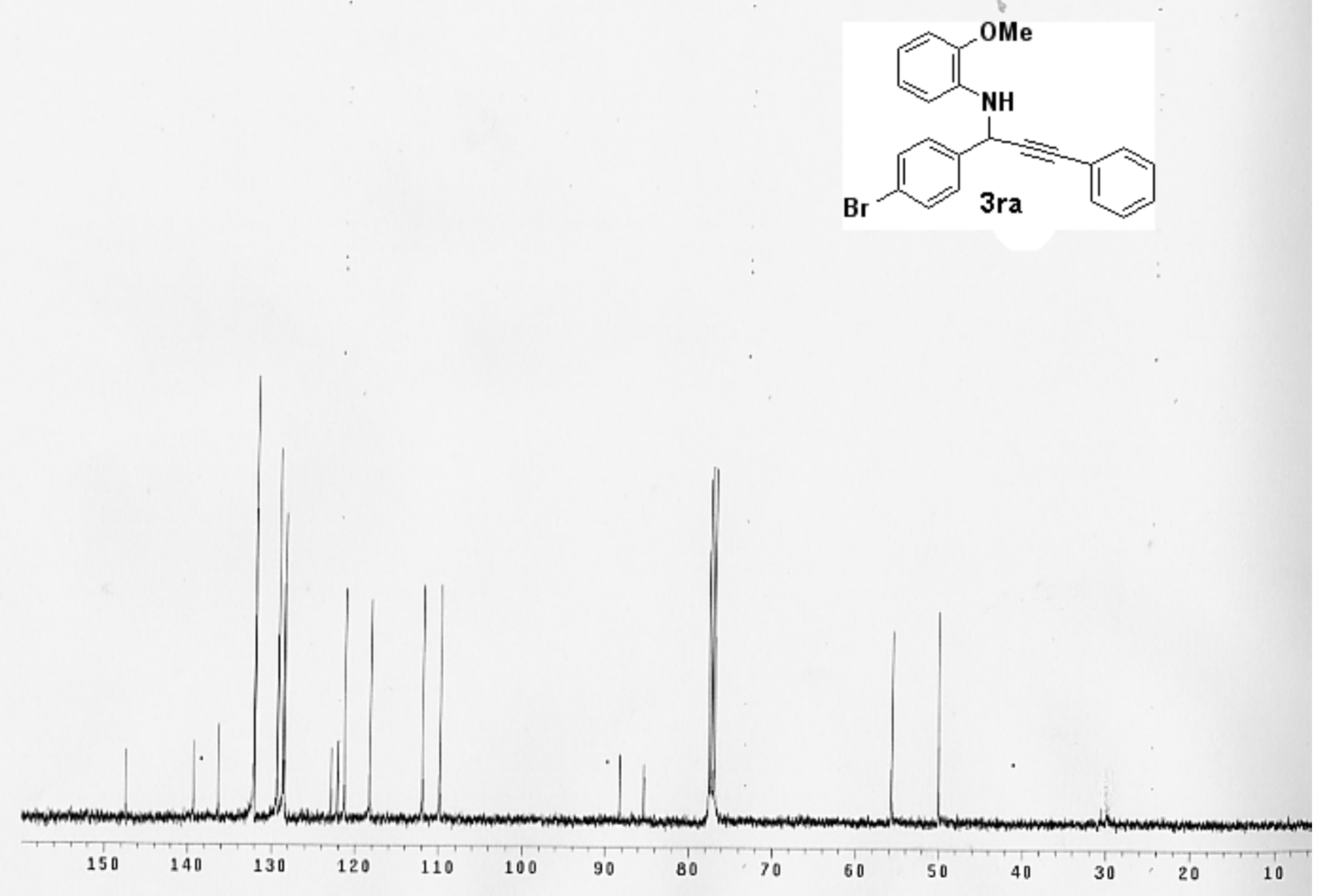


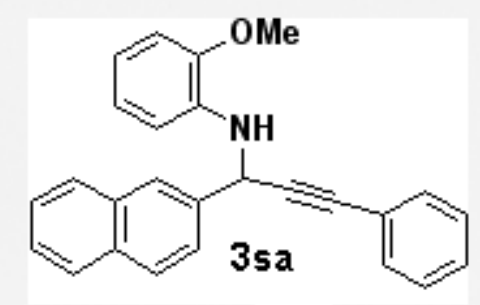




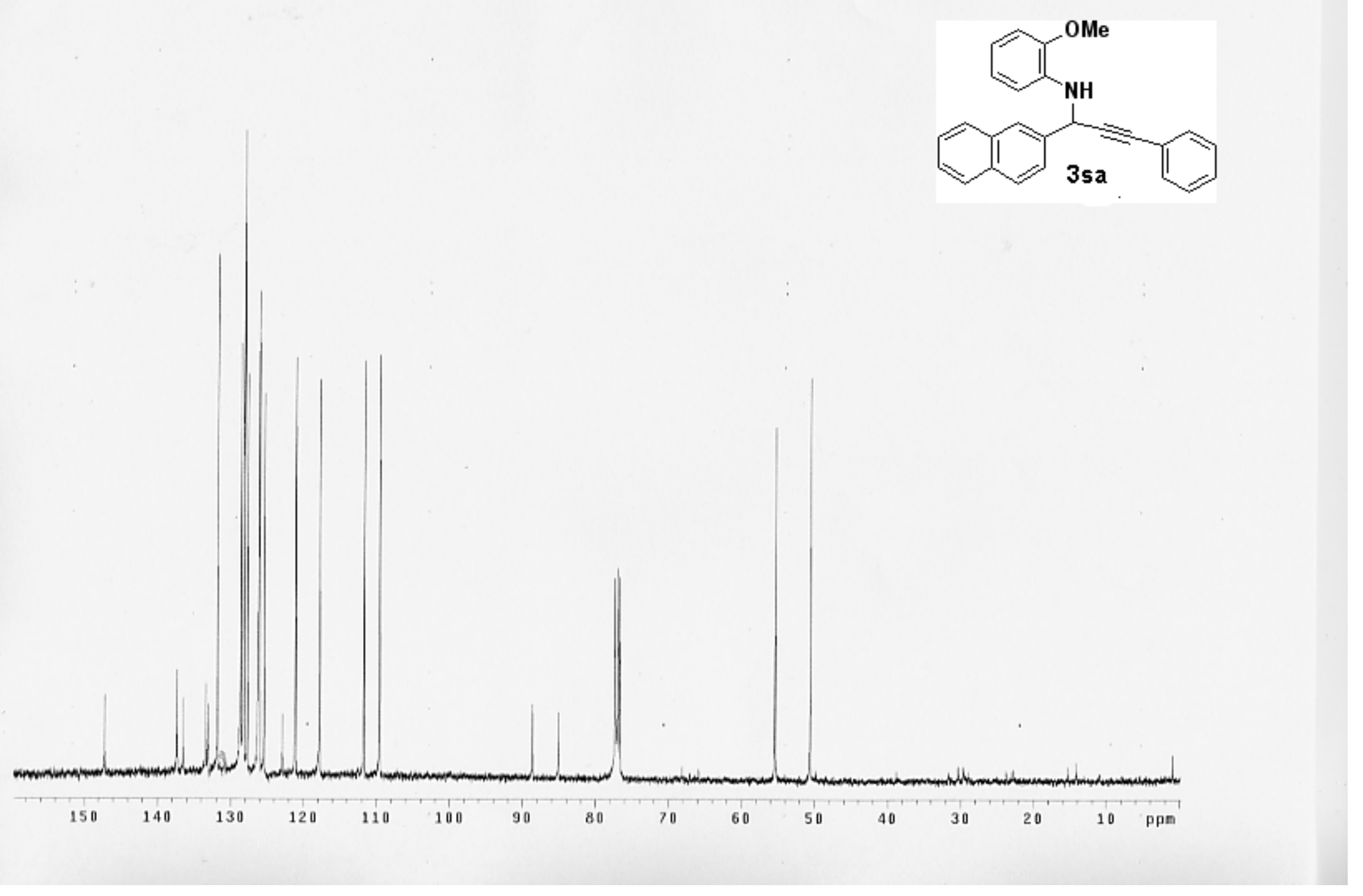




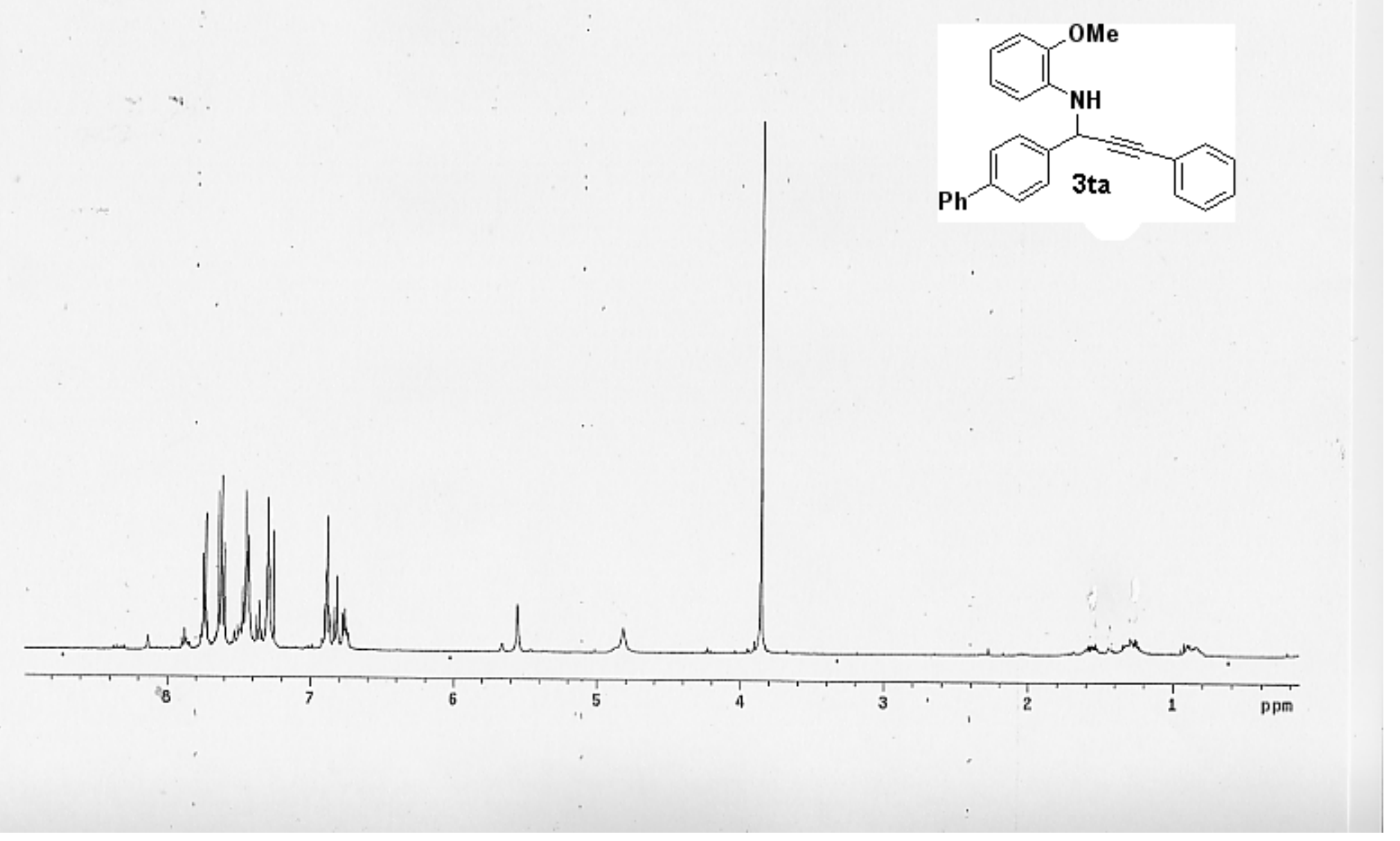




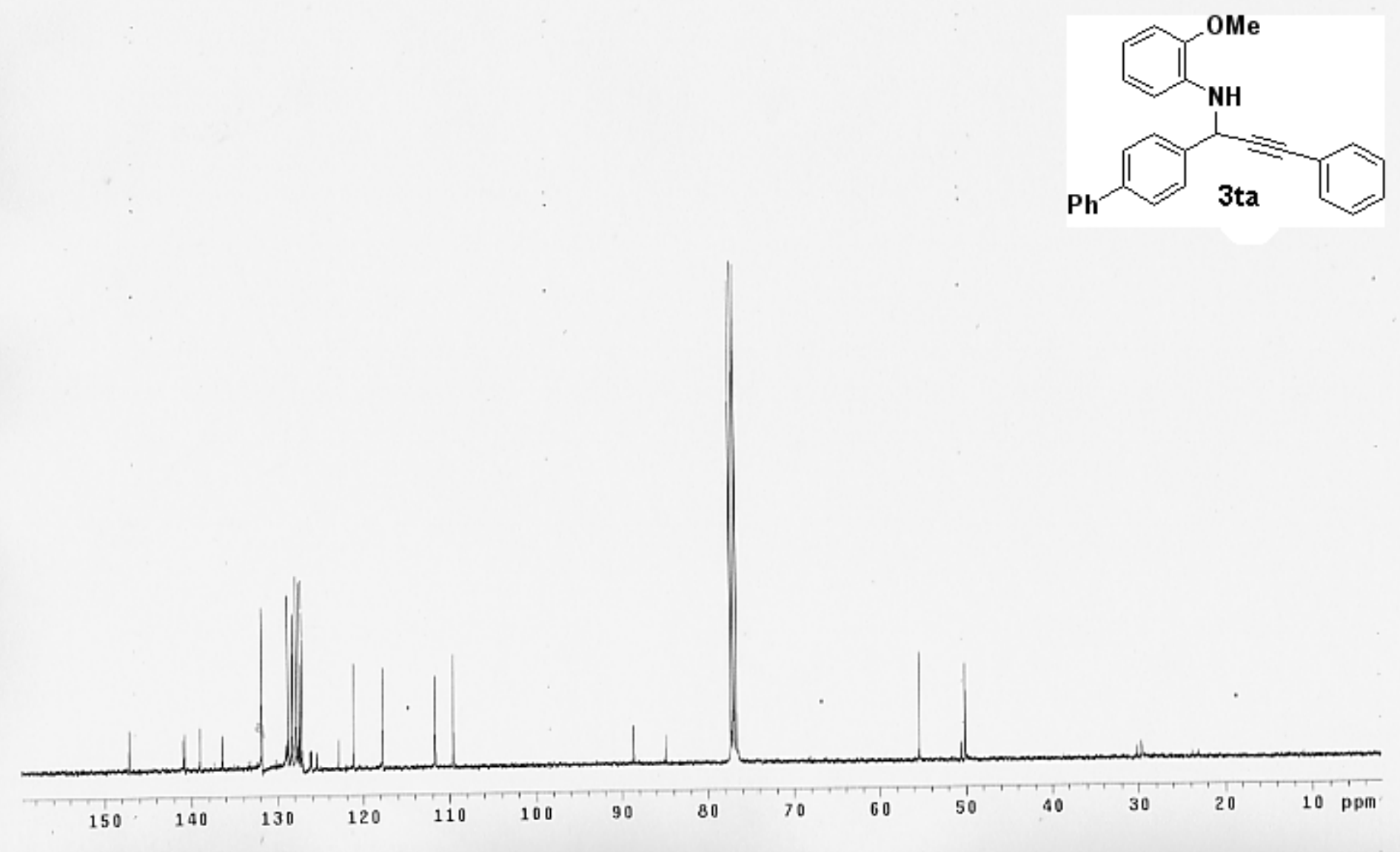




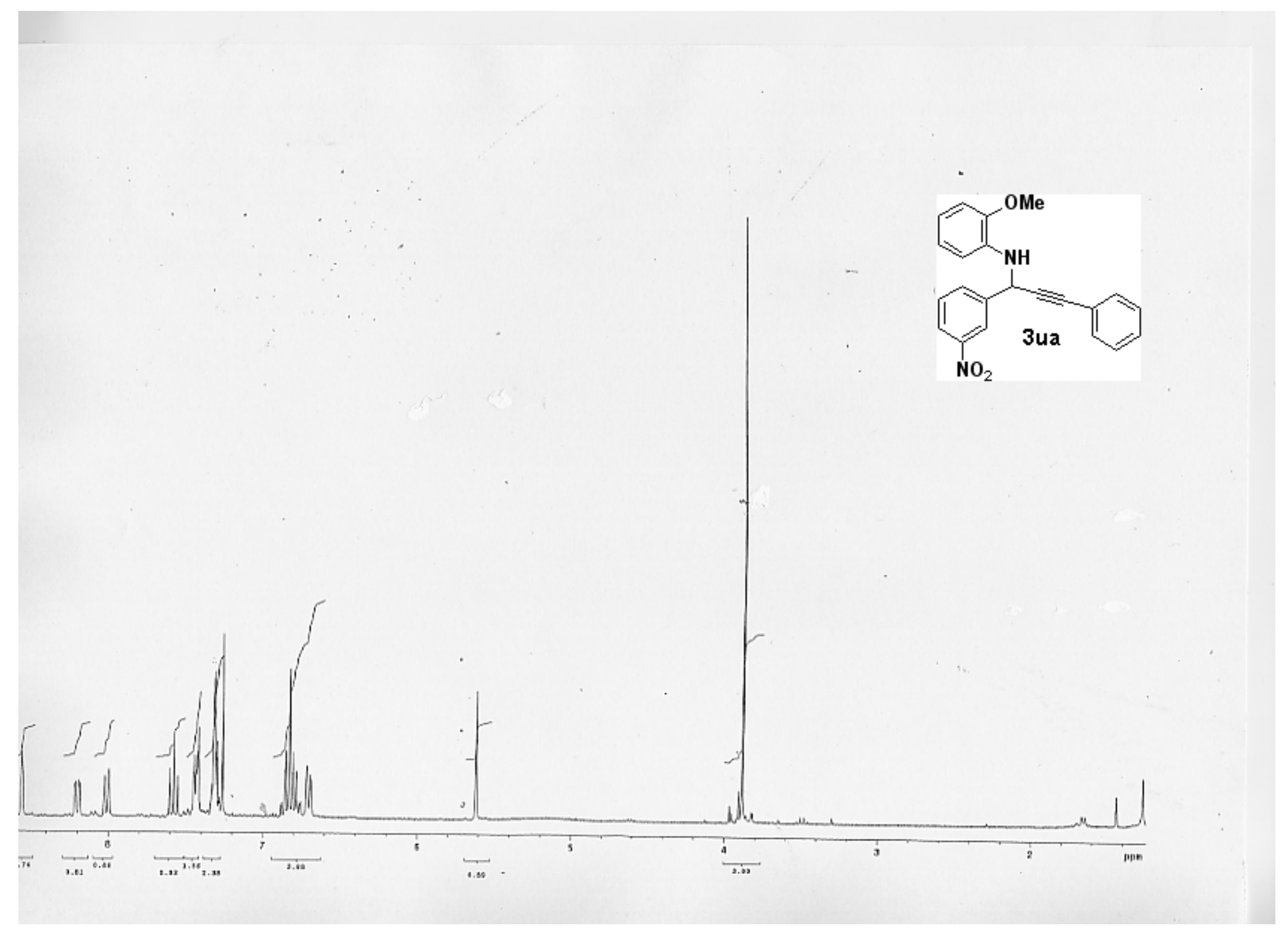




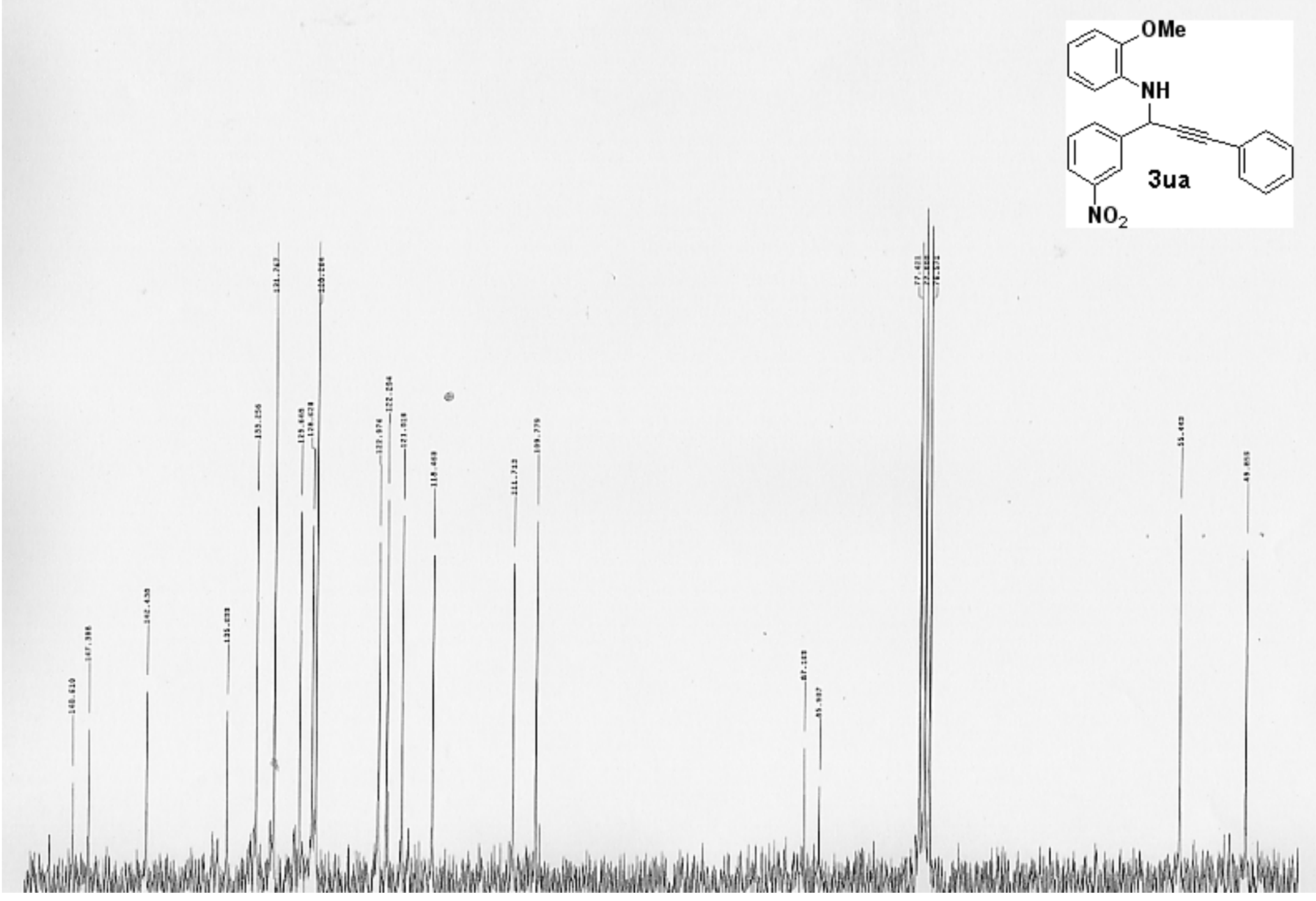


S60

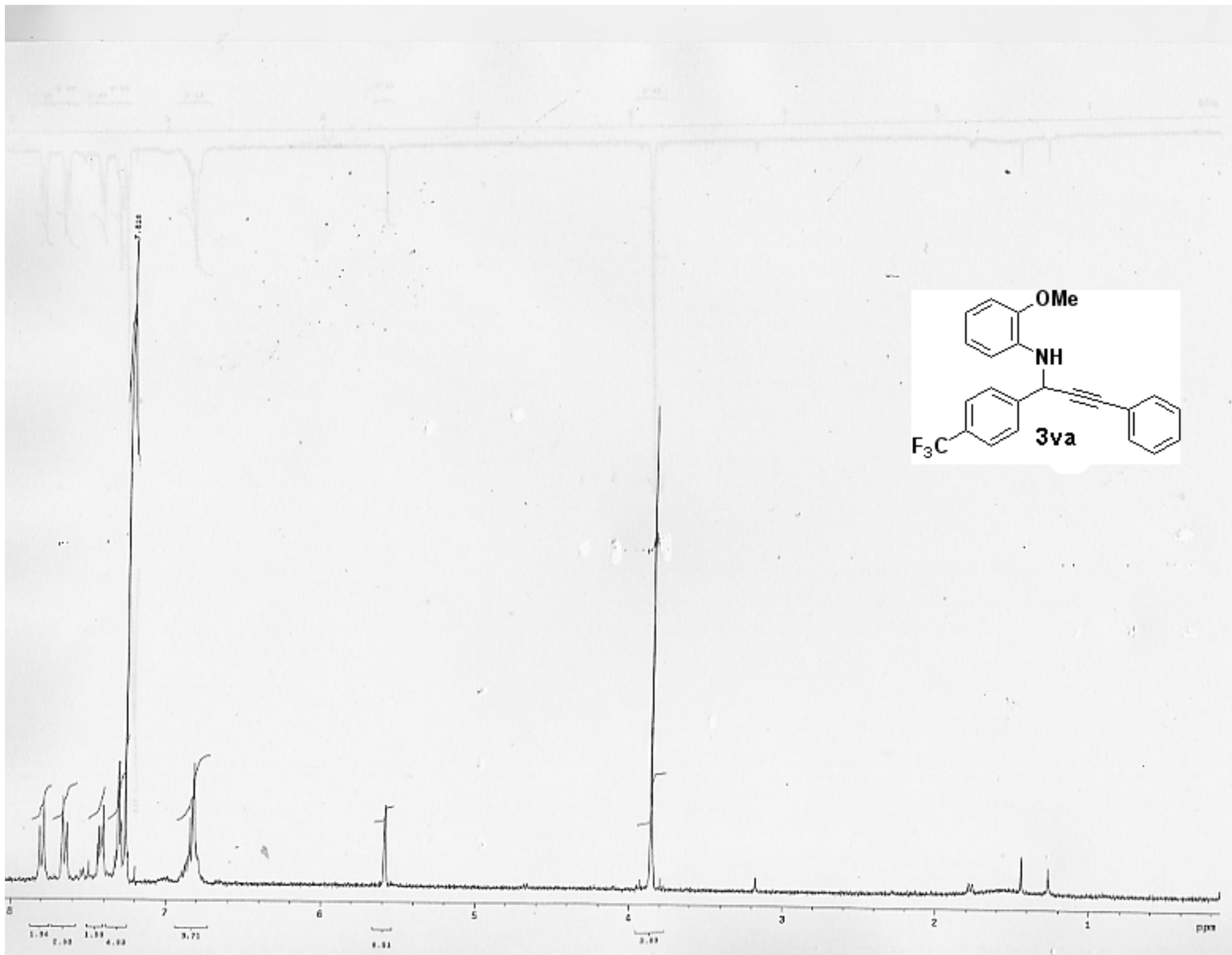

S60 


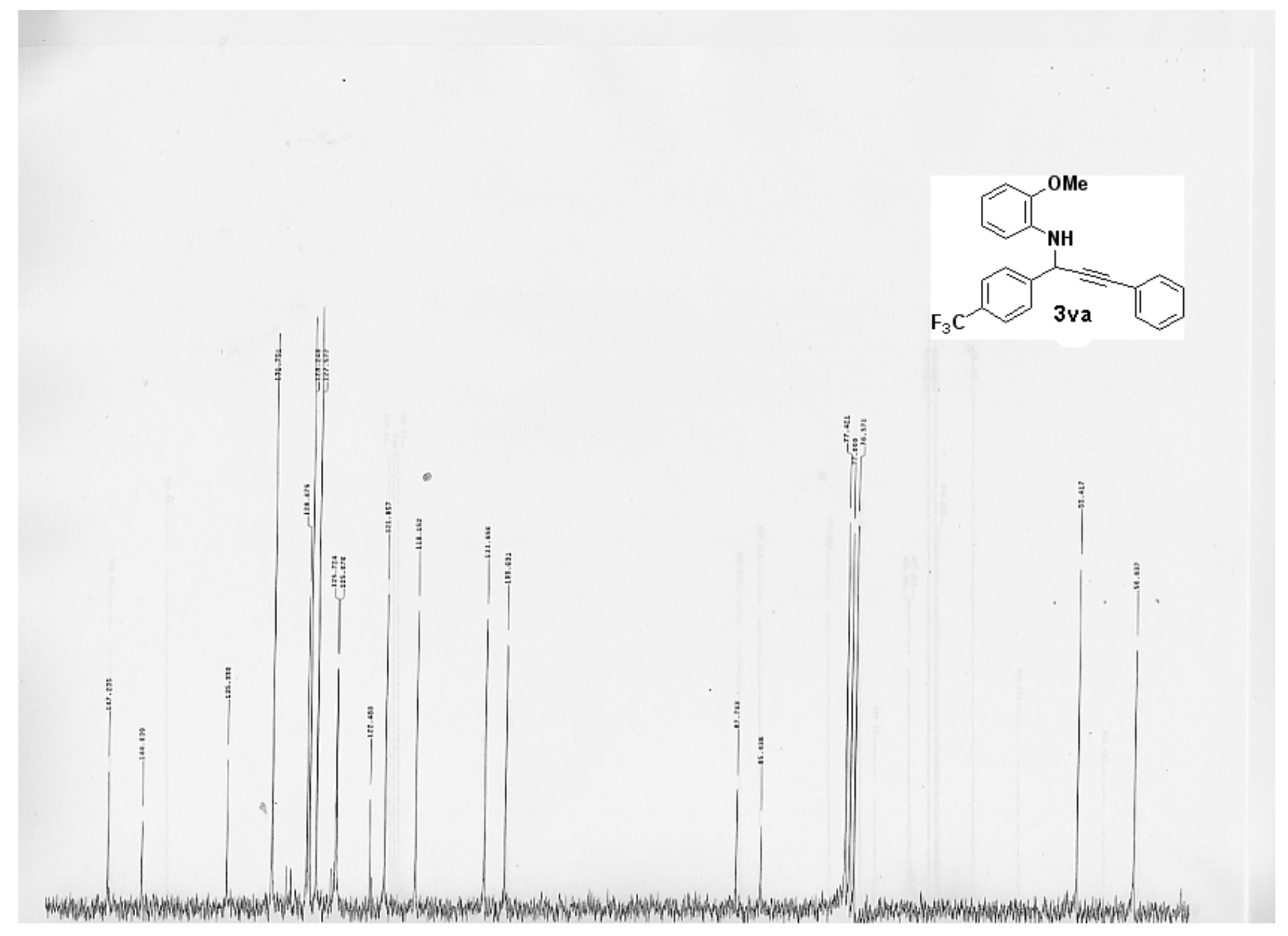




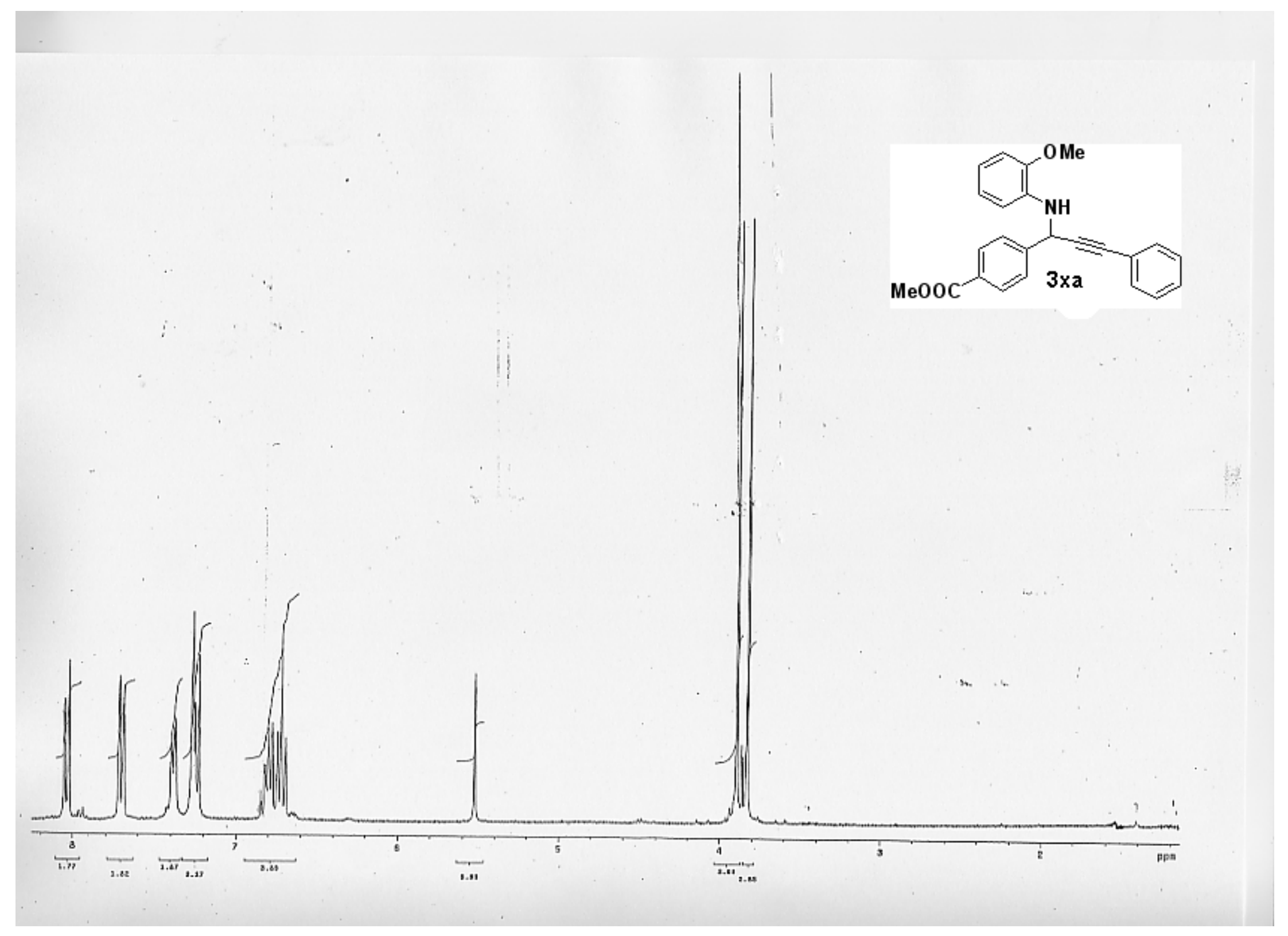


S63<smiles>COc1ccccc1NC(C#Cc1ccccc1)c1ccc(C(C)=O)cc1</smiles>

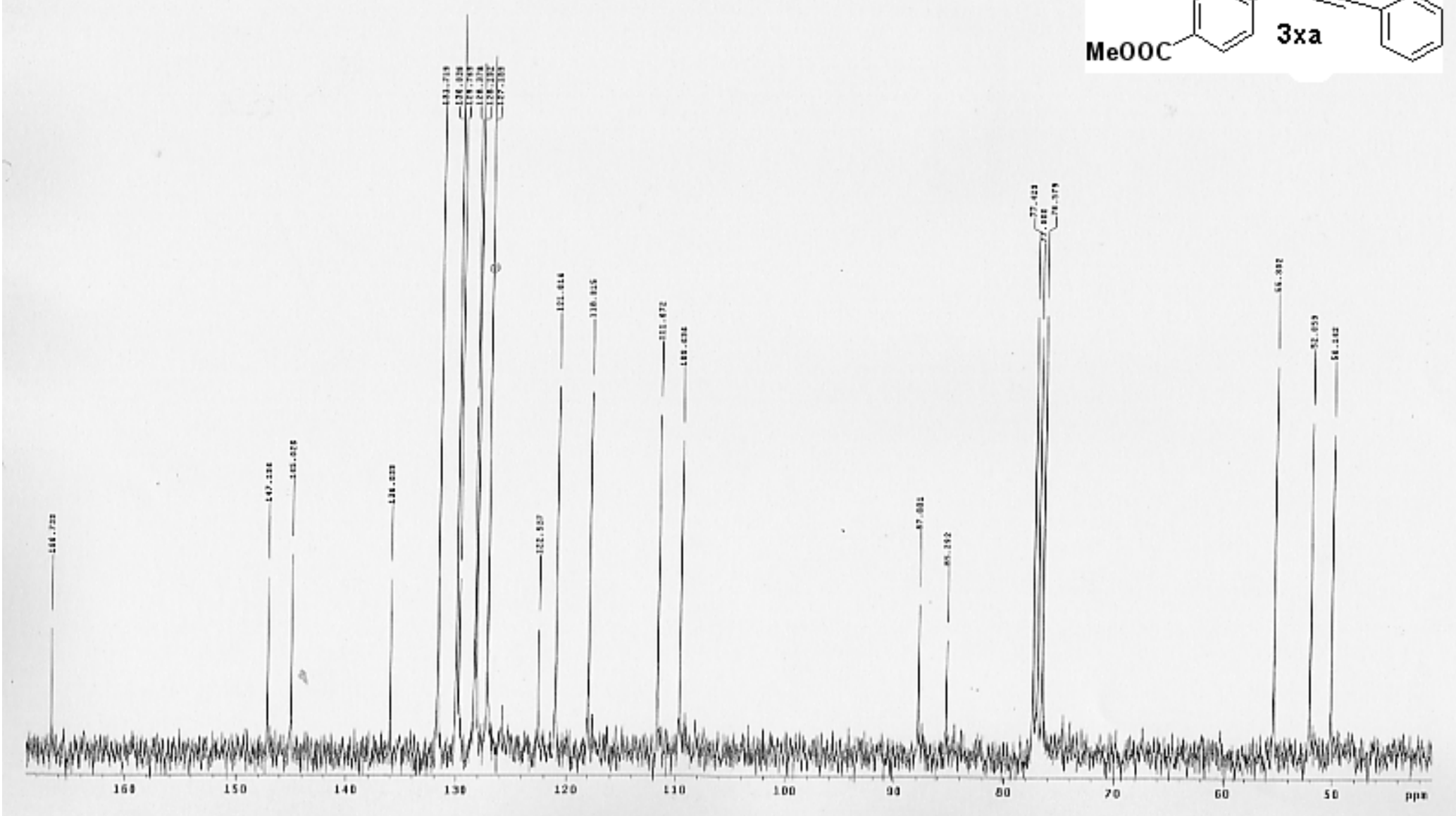

S63 


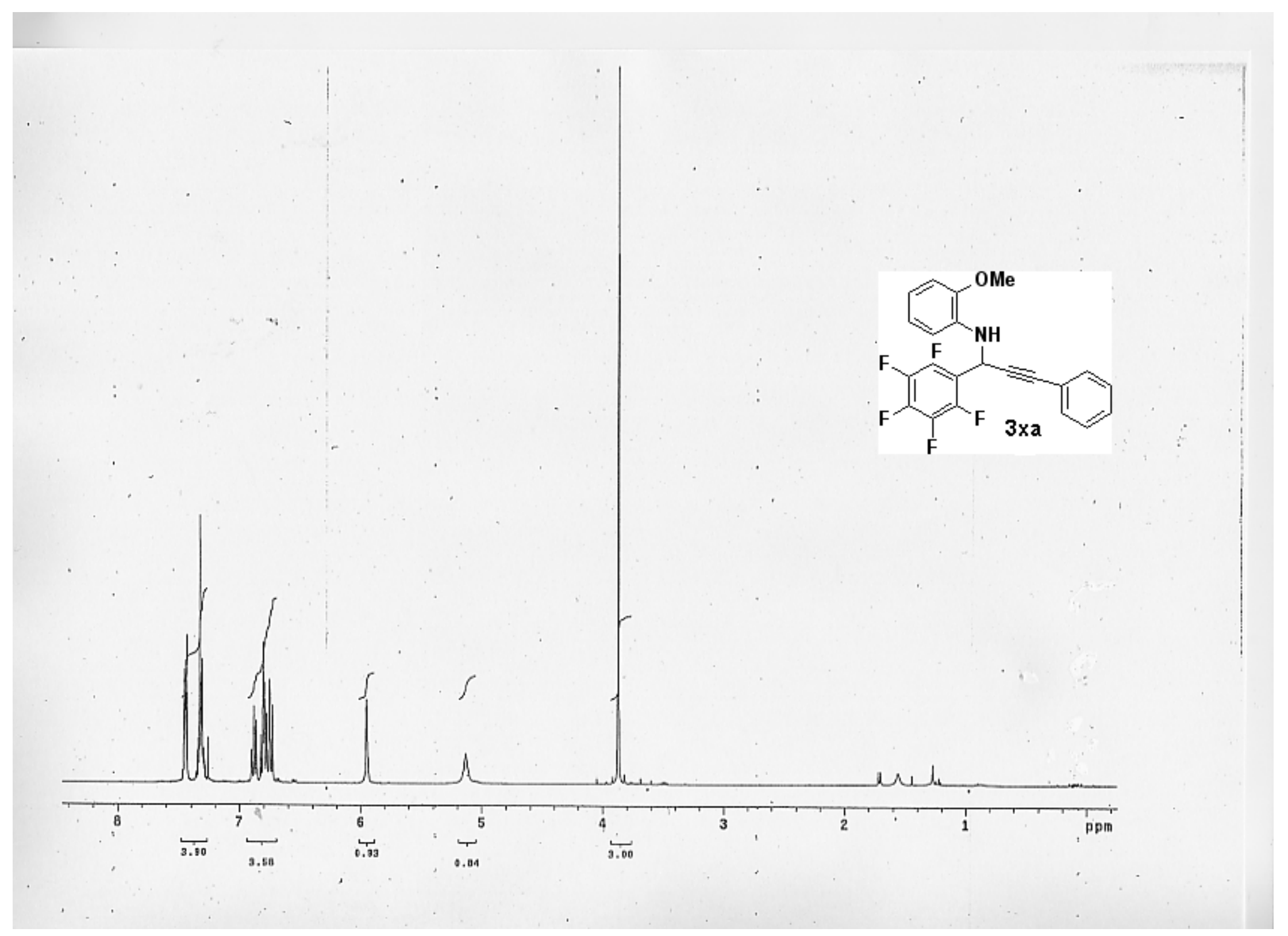





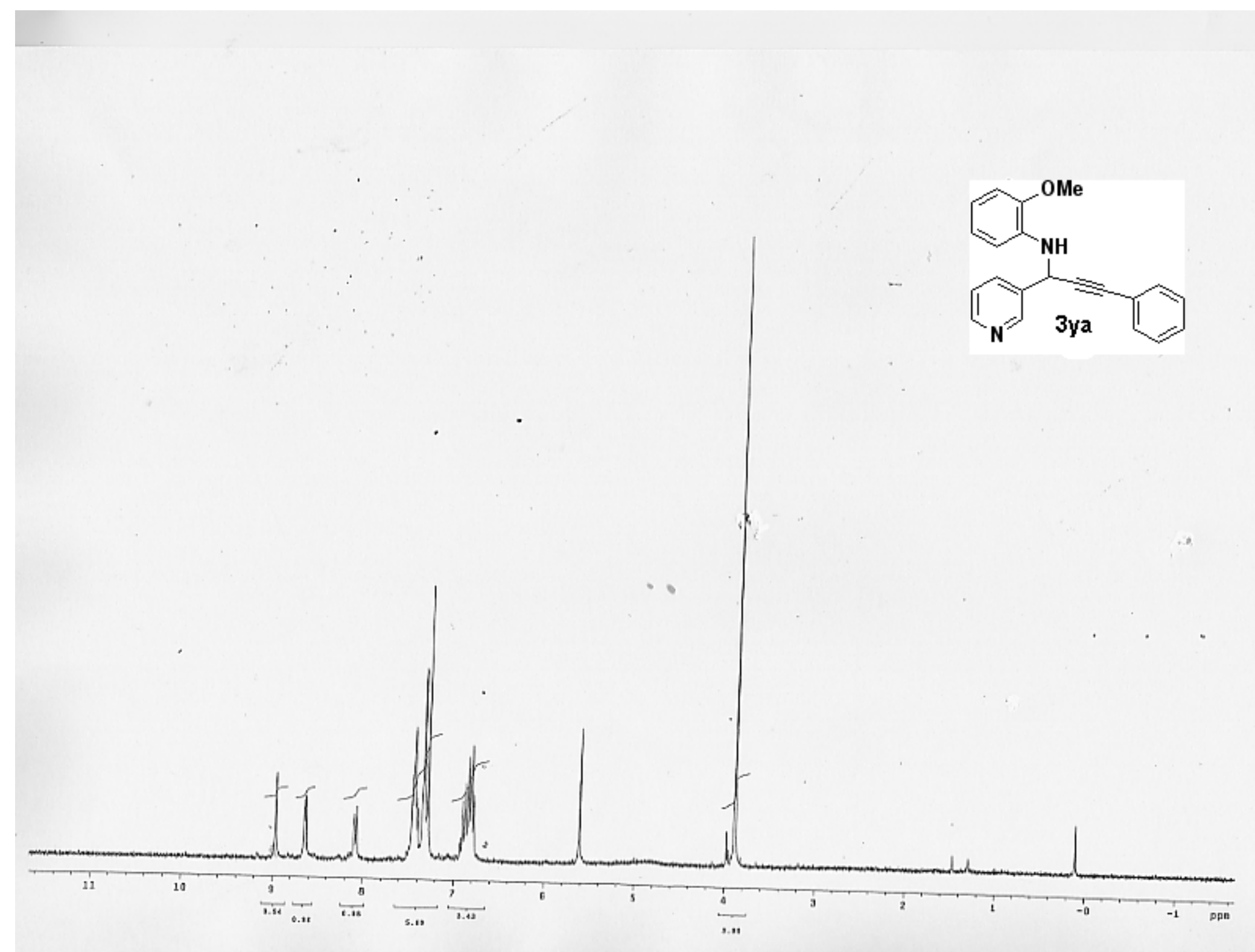




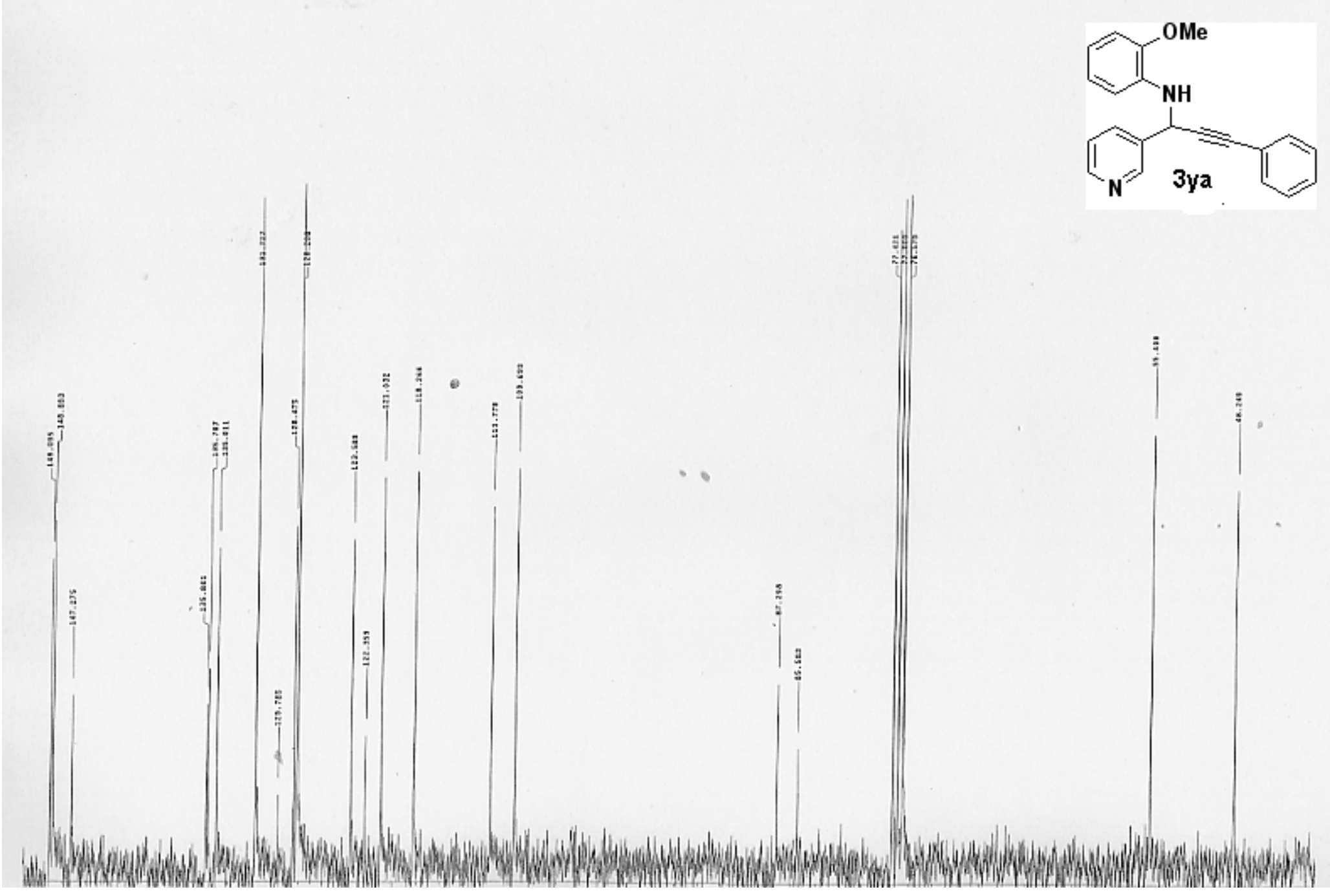


S68<smiles>COc1ccccc1NC(C#Cc1ccccc1)C1CCCCC1</smiles>

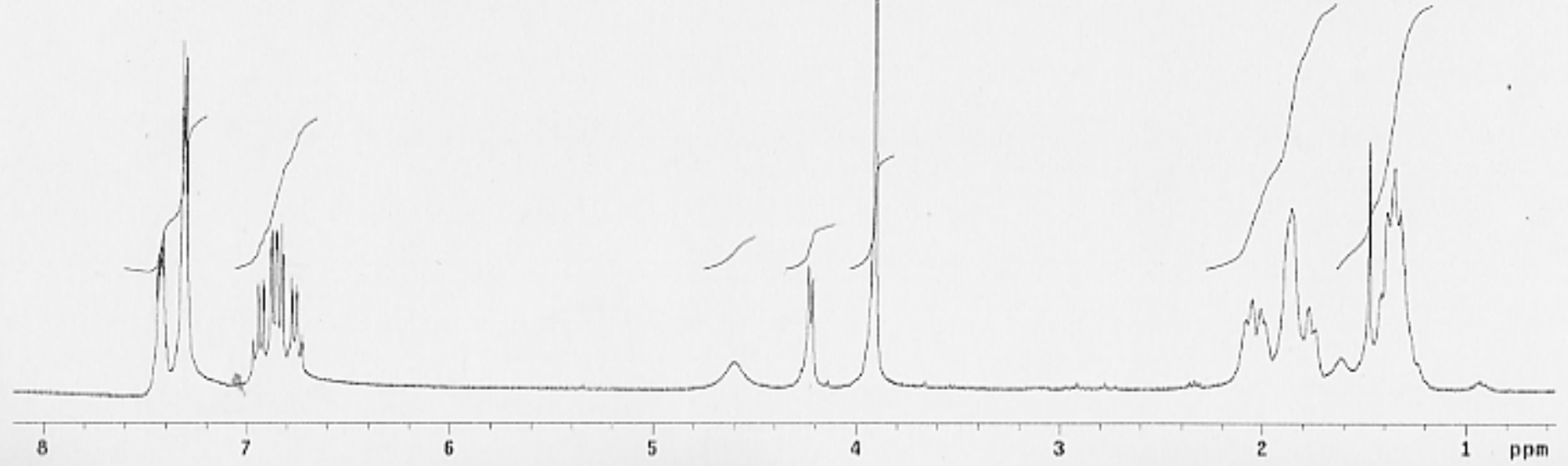

S68 


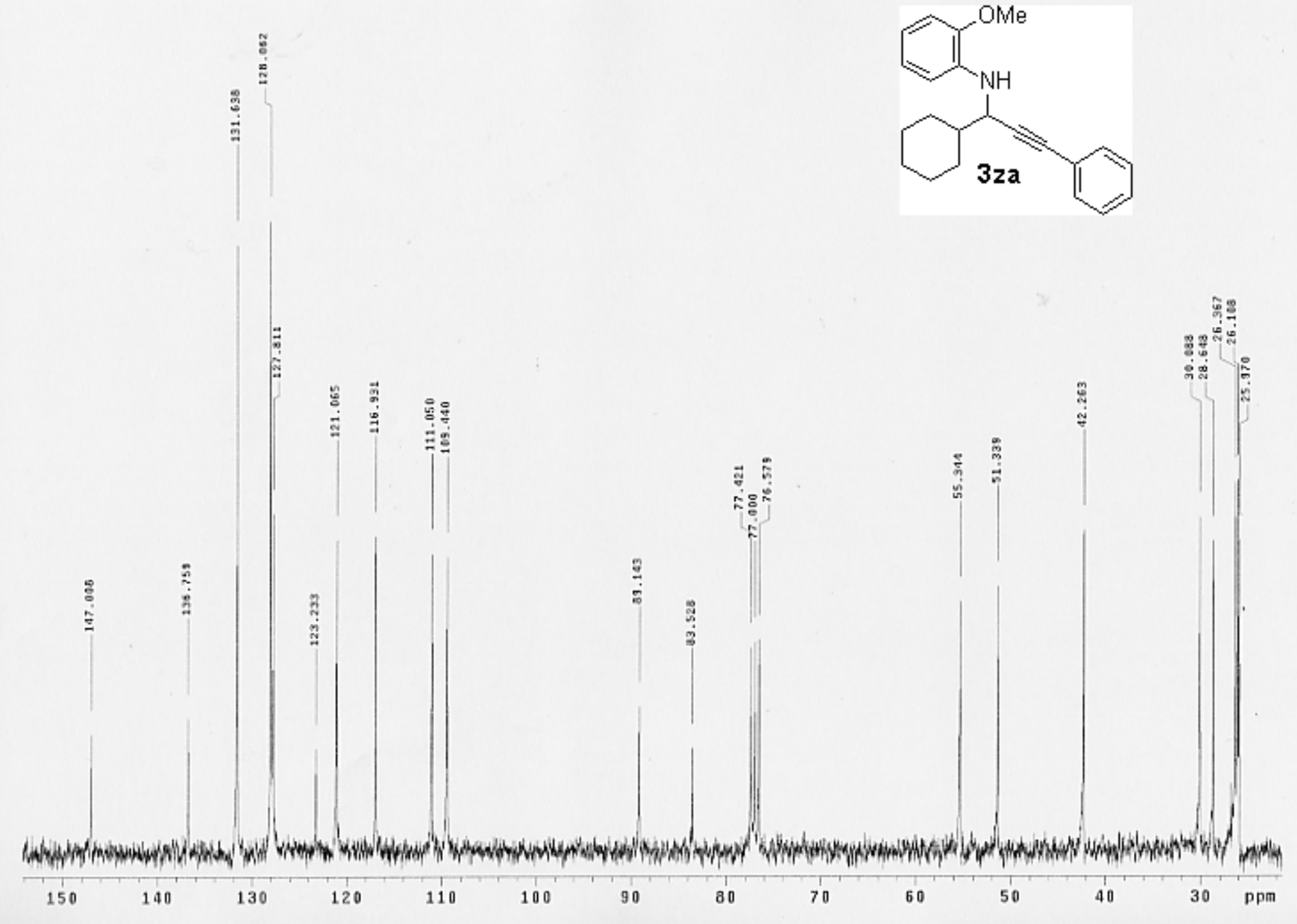


S70<smiles>COc1ccccc1NC(C#Cc1ccccc1)C(C)(C)C</smiles>

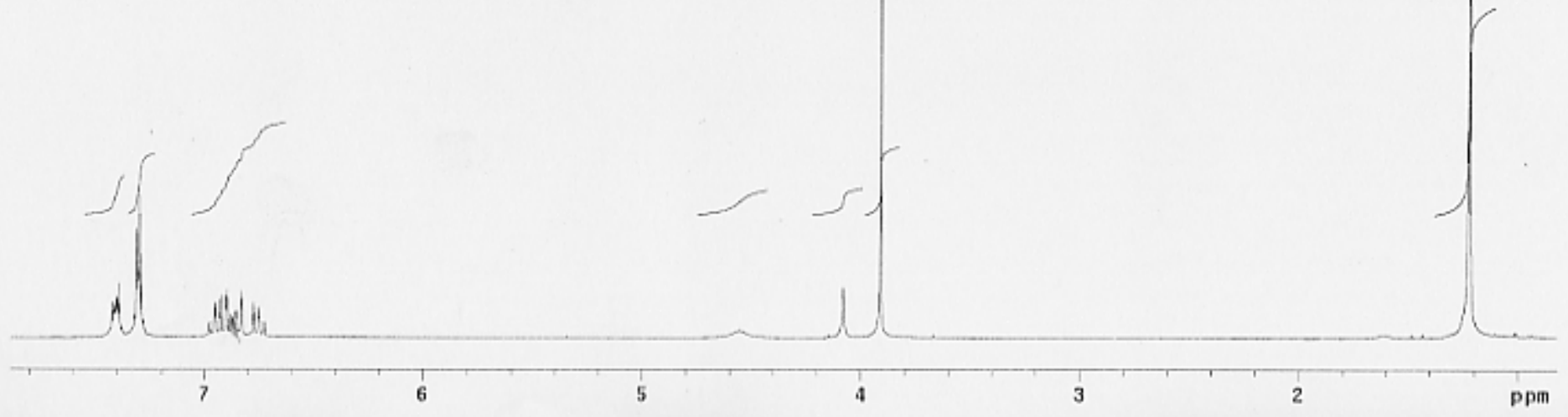

S70 


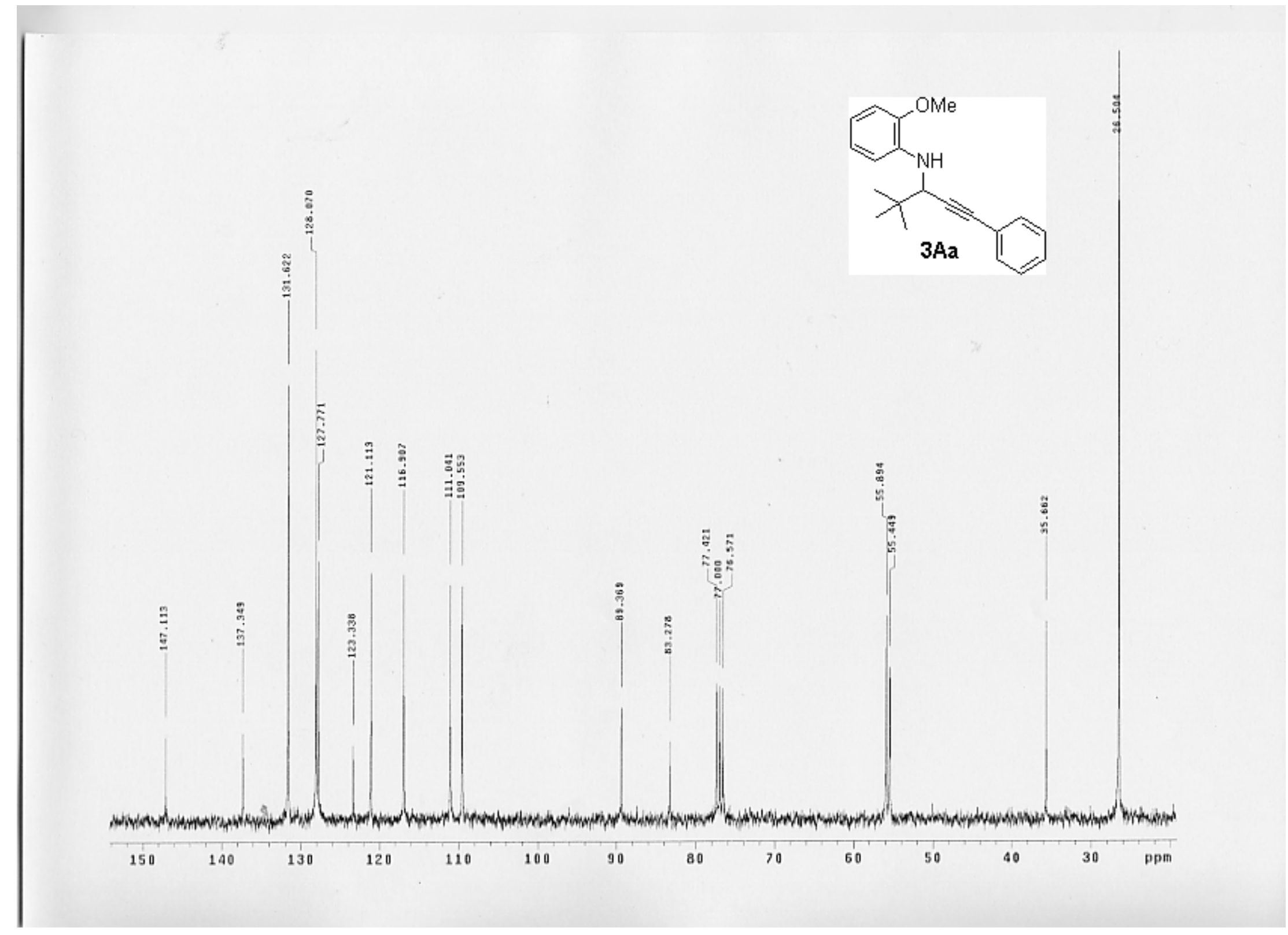




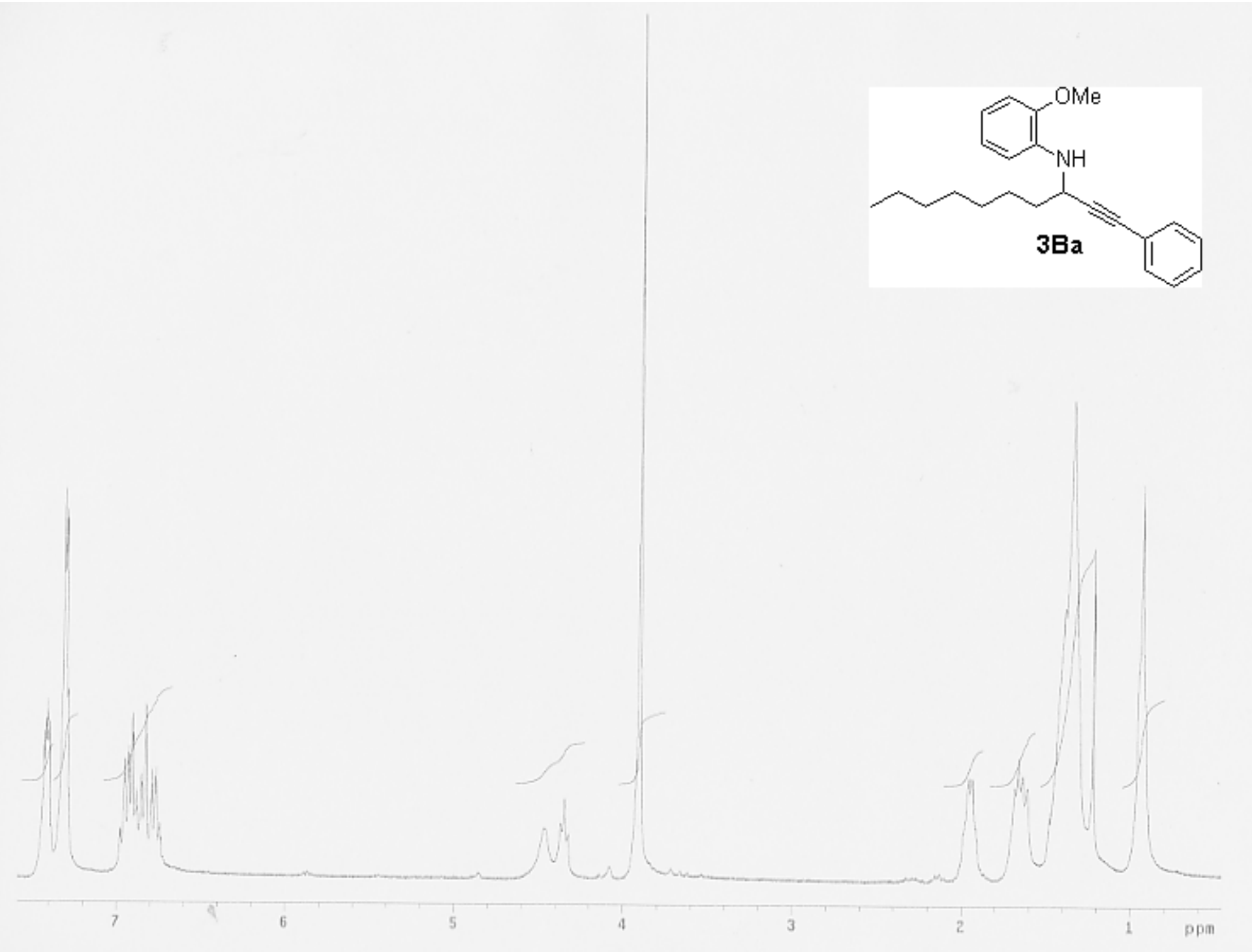




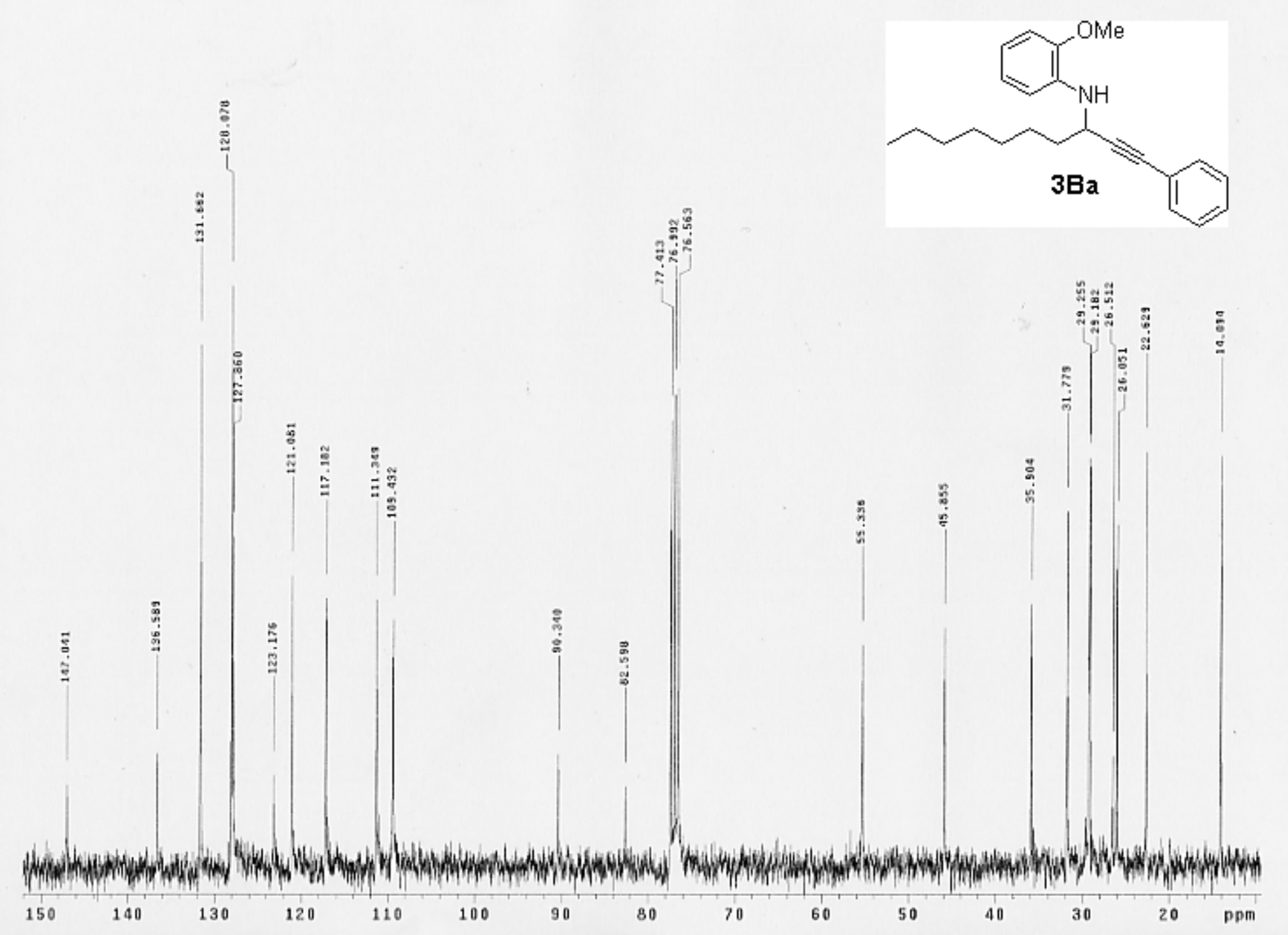

